\title{
Synthesis and conjugation of oligosaccharide analogues of fragments of the immunoreactive glycan part of the circulating anodic antigen of the parasite Schistosoma mansoni
}

\author{
Adriana Carvalho de Souza, Joeri Kuil, C. Elizabeth P. Maljaars, Koen M. Halkes, \\ Johannes F. G. Vliegenthart and Johannis P. Kamerling* \\ Bijvoet Center, Department of Bio-Organic Chemistry, Section of Glycoscience and Biocatalysis \\ Utrecht University, Padualaan 8, NL-3584 CH Utrecht, The Netherlands. \\ E-mail: j.p.kamerling@chem.uu.nl
}

Received 6th July 2004, Accepted 16th August 2004

First published as an Advance Article on the web 22nd September 2004

\begin{abstract}
The gut-associated circulating anodic antigen (CAA) is one of the major excretory antigens produced by the parasite Schistosoma mansoni. The immunoreactive part of CAA is a threonine-linked polysaccharide composed of long stretches of the unique repeating disaccharide $\rightarrow 6)-[\beta-\mathrm{D}-\mathrm{Glc} p \mathrm{~A}-(1 \rightarrow 3)]-\beta-\mathrm{D}-\mathrm{Gal} p \mathrm{NAc}-(1 \rightarrow$. Previously, using surface plasmon resonance and ELISA techniques, it has been shown that some anti-CAA IgM monoclonal antibodies (MAbs) also recognize members of a series of bovine serum albumin (BSA)-coupled synthetic di- to penta-saccharide fragments of the CAA glycan. To generate information on the molecular level about the glycan specificity of the relevant IgM MAbs, two series of oligosaccharides related to the CAA disaccharide epitope were synthesized, and coupled to BSA. The first three analogues, $\beta$-D-Glc $p \mathrm{~A}-(1 \rightarrow 3)-\beta$-D-Glc $p$ NAc- $(1 \rightarrow \mathrm{O}), \beta$-D-Glc $p$ NAc$(1 \rightarrow 6)-[\beta$-D-Glc $p \mathrm{~A}-(1 \rightarrow 3)]-\beta$-D-Glc $p$ NAc- $(1 \rightarrow \mathrm{O})$, and $\beta$-D-Glc $p \mathrm{~A}-(1 \rightarrow 3)-\beta$-D-Glc $p \mathrm{NAc}-(1 \rightarrow 6)-[\beta$-D-Glc $p \mathrm{~A}-(1 \rightarrow$ 3)]- $\beta$-D-Glc $p$ NAc- $(1 \rightarrow \mathrm{O})$, wherein the native $\beta$-D-Gal $p$ NAc moiety was replaced by $\beta$-D-Glc $p$ NAc, were synthesized to investigate the specificity of the selected MAbs to the carbohydrate backbone of CAA. The second series of analogues, $\beta$-D-Glc $p 6 S$ - $(1 \rightarrow 3)-\beta$-D-Gal $p$ NAc- $(1 \rightarrow \mathrm{O}), \beta$-D-Gal $p$ NAc- $(1 \rightarrow 6)-[\beta$-D-Glc $p 6 S-(1 \rightarrow 3)]-\beta$-D-Gal $p$ NAc- $(1 \rightarrow$ $\mathrm{O})$, and $\beta$-D-Glcp $6 S$ - $(1 \rightarrow 3)-\beta$-D-Gal $p$ NAc- $(1 \rightarrow 6)-[\beta$-D-Glcp $6 S-(1 \rightarrow 3)]-\beta$-D-Gal $p$ NAc- $(1 \rightarrow \mathrm{O})$, wherein the native $\beta$ D-GlcpA moiety was replaced by $\beta$-D-Glcp $6 S$, was synthesized to evaluate the importance of the type/nature of the charge of CAA for the MAb recognition.
\end{abstract}

\section{Introduction}

One of the most prevalent tropical diseases is schistosomiasis, otherwise known as bilharzia, which is caused by a parasitic blood fluke of the genus Schistosoma. The intriguing and complex life cycle of this worm involves several parasitic stages in the intermediate (fresh-water snails) and definitive host, alternated by two larvae stages. ${ }^{1}$ Owing to the regional occurrence of the intermediate host, the disease is limited to tropical and subtropical areas, where an estimated 200 million people are infected and suffer from the debilitating effects of this disease. ${ }^{2}$ The control strategies against schistosomiasis are normally built on the results of diagnosic tests. The microscopic demonstration of the parasite's eggs on faeces or in urine is the more widespread tool for the diagnosis of Schistosoma infections in epidemic areas.

In recent years a variety of immunological techniques have been described in the literature as alternative techniques to faecal or urinary egg counts. ${ }^{3-6}$ An early and strong humoral immune response to schistosomes is directed to glycan epitopes of a number of circulating antigens, in particular the gut-associated circulating anodic antigen (CAA) and circulating cathodic antigen (CCA). ${ }^{3}$ Moreover, several studies have demonstrated a strong correlation between antigen levels and the number of adult worms, ${ }^{7,8}$ and that antigen levels decreased rapidly following successful chemotherapy. ${ }^{8}$ For the assessment of a cure and for the diagnosis of active infections in endemic areas, the method of choice is the determination of CAA or CCA in the serum or urine of infected subjects. ${ }^{6}$ So far, a specificity of $\bar{z}$ virtually $100 \%$ was found at the ELISA demonstration of CAA in serum, while for CCA, false positive results were occasionally observed. ${ }^{9}$

The major immunogenic character of CAA is carried by an O-linked polysaccharide chain composed of the unique disaccharide repeating unit $\rightarrow 6)-[\beta-D-G l c p A-(1 \rightarrow 3)]-\beta-D-$ GalpNAc- $\left(1 \rightarrow .{ }^{10}\right.$ The uniqueness of the primary structure of this polysaccharide chain may be responsible for the absolute specificity of the CAA immunodiagnostic assays. In a previous study, a panel of monoclonal antibodies (MAbs) raised against S. mansoni adult worm antigens was screened for recognition of synthetic di- to penta-saccharide fragments of the CAA polysaccharide, multivalently presented as bovine serum albumin (BSA) conjugates. ${ }^{11,12}$ The results showed that several MAbs, especially of the IgM class, already recognized the disaccharide unit $\beta$-DGlcpA- $(1 \rightarrow 3)-\beta$-D-Gal $p$ NAc- $(1 \rightarrow \mathrm{O})$. In order to understand in molecular detail the specificity of the anti-carbohydrate MAbs, we synthesized two series of structures (Fig. 1) related to the CAA epitope, and conjugated these oligosaccharides to BSA, using squaric diester chemistry. The first series of synthetic analogues, $\beta$-D-Glc $p \mathrm{~A}-(1 \rightarrow 3)-\beta-\mathrm{D}-\mathrm{Glc} p \mathrm{NAc}-(1 \rightarrow \mathrm{O})(\mathbf{1}), \beta$-DGlc $p$ NAc- $(1 \rightarrow 6)-[\beta-\mathrm{D}-\mathrm{Glc} p \mathrm{~A}-(1 \rightarrow 3)]-\beta-\mathrm{D}-\mathrm{Glc} p \mathrm{NAc}-(1 \rightarrow \mathrm{O})$ $(2)$, and $\beta$-D-Glc $p A-(1 \rightarrow 3)-\beta-D-G l c p N A c-(1 \rightarrow 6)-[\beta-D-G l c p A-$ $(1 \rightarrow 3)]-\beta$-D-Glc $p$ NAc- $(1 \rightarrow \mathrm{O})(3)$, has the native $\beta$-D-Gal $p$ NAc residue replaced by $\beta-\mathrm{D}-\mathrm{Glc} p$ NAc to evaluate the importance of the hydroxyl function HO4 in the carbohydrate backbone for the MAb recognition. In the other series of structures, $\beta$-D-Glc $p 6 S$ - $(1 \rightarrow 3)-\beta$-D-Gal $p$ NAc- $(1 \rightarrow \mathrm{O}) \quad(4), \quad \beta$-D-Gal $p$ NAc$(1 \rightarrow 6)-[\beta$-D-Glcp $6 S-(1 \rightarrow 3)]-\beta$-D-Gal $p N A c-(1 \rightarrow \mathrm{O}) \quad(5), \quad$ and $\beta$-D-Glc $p 6 S-(1 \rightarrow 3)-\beta$-D-Galp NAc- $(1 \rightarrow 6)-[\beta-D-G 1 c p 6 S-(1 \rightarrow$ 3)]- $\beta$-D-Gal $p$ NAc- $(1 \rightarrow \mathrm{O})(6)$, the native $\beta$-D-Glc $p$ A moiety was replaced by $\beta$-D-Glcp $6 S$ in order to investigate the influence of the nature of charge on the $\mathrm{MAb}$ recognition.

\section{Results and discussion}

Synthesis of oligosaccharide analogues $1-3$ containing $\beta$-DGlcpNAc instead of $\beta$-D-GalpNAc moieties

In the synthetic routes to target oligosaccharides $\mathbf{1}-\mathbf{3}$, four earlier reported monosaccharide building blocks were used, namely, $7,8,11$, and $\mathbf{1 4}$. In contrast to our earlier approach of preparing fragments of the native CAA glycan, which included a 


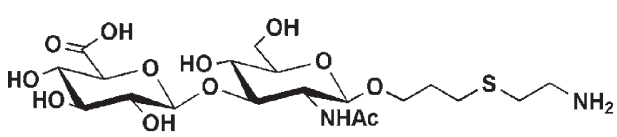

1
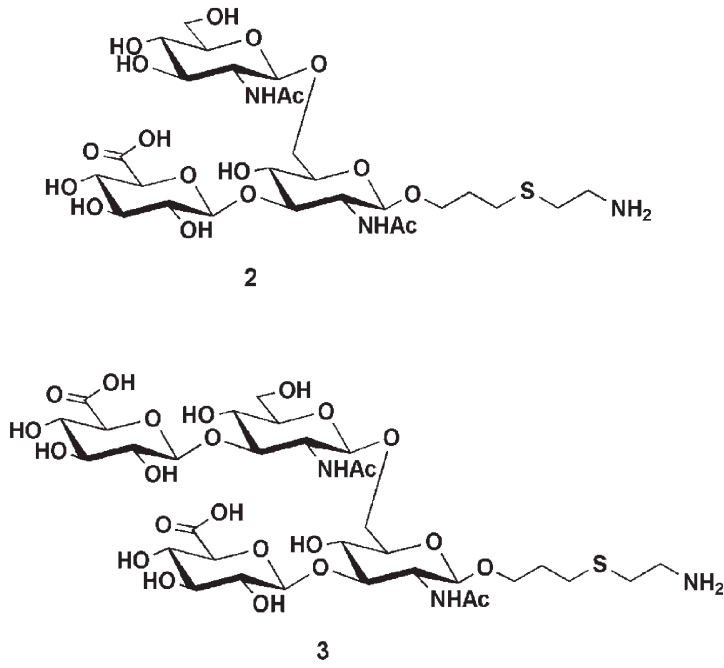
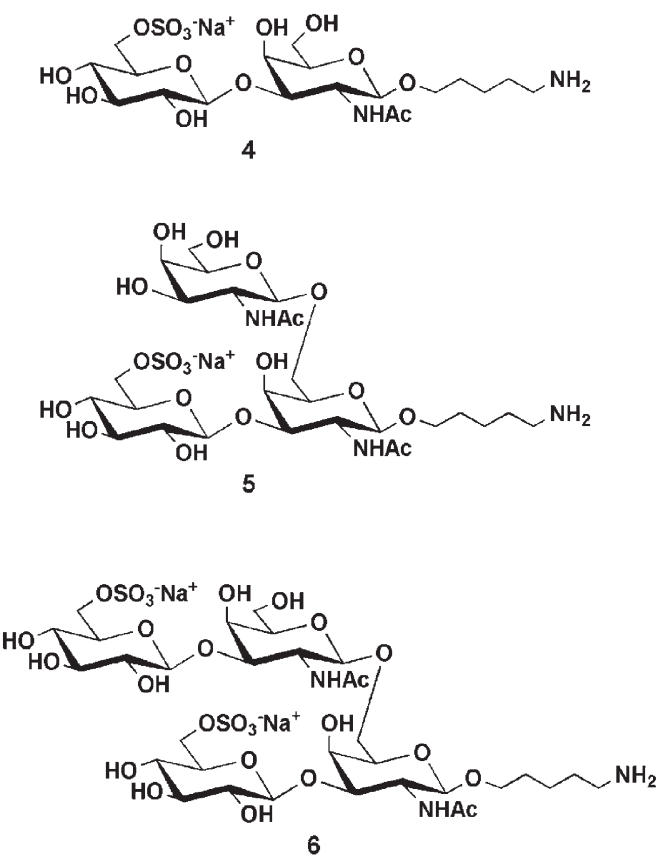

Fig. 1 Target oligosaccharides 1-6.

time-consuming C6 oxidation step at later stages of the synthesis to generate glucuronic acid units, here, methyl 2,3,4-tri- $O$-acetyl$\alpha, \beta$-D-glucopyranosyluronate trichloroacetimidate $\mathbf{8}$ has been chosen.

For the synthesis of disaccharide 1, acceptor allyl 2-deoxy4,6- $O$-isopropylidene-2-phthalimido- $\beta$-D-glucopyranoside $7^{13}$ was coupled with donor methyl 2,3,4-tri- $O$-acetyl- $\alpha, \beta$-D-glucopyranosyluronate trichloroacetimidate $\mathbf{8},{ }^{14}$ using trimethylsilyl triflate as a promoter (Scheme 1). After removal of the isopropylidene group under acidic conditions, ${ }^{15}$ disaccharide 9 was obtained in a moderate yield (31\%). The main side reaction during the condensation is the formation of an orthoester intermediate that could not be completely converted into the desired product. Alternative attempts, such as using different temperatures, promoter concentrations, and protecting groups for the donor, did not improve the yield. For deprotection of the disaccharide 9, the methyl ester and acetyl groups were saponified with $3 \mathrm{M}$ aq. $\mathrm{NaOH}$ in 5:1 methanol-water. ${ }^{14}$ Subsequent dephthaloylation was carried out with 1,2-diaminoethane in $n$-butanol at $90{ }^{\circ} \mathrm{C},{ }^{16}$ and the formed product was $N$ acetylated with acetic anhydride in methanol at $0{ }^{\circ} \mathrm{C}^{17}$ to give the fully deprotected allyl glycoside $\mathbf{1 0}(52 \%)$. Finally, $\mathbf{1 0}$ was reacted with cysteamine hydrochloride ${ }^{18}$ under radical conditions (UVirradiation) to afford the amino-spacer-containing disaccharide $1(76 \%)$.

For the synthesis of trisaccharide 2, 3,4,6-tri- $O$-acetyl-2deoxy-2-phthalimido- $\beta$-D-glucopyranosyl trichloroacetimidate $11^{19}$ was regioselectively-coupled with disaccharide acceptor 9, at $0{ }^{\circ} \mathrm{C}$, using trimethylsilyl triflate as a promoter $(\rightarrow \mathbf{1 2}$,
$86 \%$; Scheme 2). Saponification of 12 with $3 \mathrm{M}$ aq. $\mathrm{NaOH}$ in $5: 1$ methanol-water, followed by dephthaloylation with 1,2-diaminoethane in $n$-butanol at $90{ }^{\circ} \mathrm{C}$, and subsequent $N$-acetylation using acetic anhydride in methanol at $0{ }^{\circ} \mathrm{C}$, rendered allyl glycoside $13(42 \%)$. Elongation of the allyl spacer of 13 with cysteamine resulted in target trisaccharide $2(48 \%)$.

For the synthesis of tetrasaccharide $\mathbf{3}$, disaccharide donor 17 was prepared (Scheme 3). Coupling of donor $8^{14}$ with acceptor 4-methoxyphenyl 2-deoxy-4,6- $O$-isopropylidene-2phthalimido- $\beta$-D-glucopyranoside $\mathbf{1 4},{ }^{20}$ using trimethylsilyl triflate as a promoter, followed by removal of the isopropylidene group under acidic conditions, gave disaccharide 15 (71\%). As mentioned already for $\mathbf{9}$, this condensation reaction also proceeds via orthoester formation and subsequent conversion into the desired adduct. The good yield obtained here is probably due to a different protection of the anomeric center of acceptor 14. After conventional acetylation of the $\mathrm{HO} 4$ and $\mathrm{HO} 6$ groups of $\mathbf{1 5}(\rightarrow \mathbf{1 6}$, quantitative), oxidative removal of the anomeric 4-methoxyphenyl group, using ammonium cerium(IV) nitrate, ${ }^{21}$ followed by imidation ${ }^{19}$ of the hemiacetal, resulted in disaccharide donor $17(63 \%)$.

Regioselective coupling of disaccharide donor 17 with disaccharide acceptor 9 (Scheme 4), using trimethylsilyl triflate as a promoter, gave tetrasaccharide 18 (90\%). Saponification of 18 with $3 \mathrm{M}$ aq. $\mathrm{NaOH}$ in 5:1 methanol-water, followed by dephthaloylation and $N$-acetylation afforded allyl glycoside 19 $(42 \%)$. Finally, 19 was elongated with cysteamine under UVlight, to obtain the amino-spacer-containing tetrasaccharide $\mathbf{3}$ $(56 \%)$.
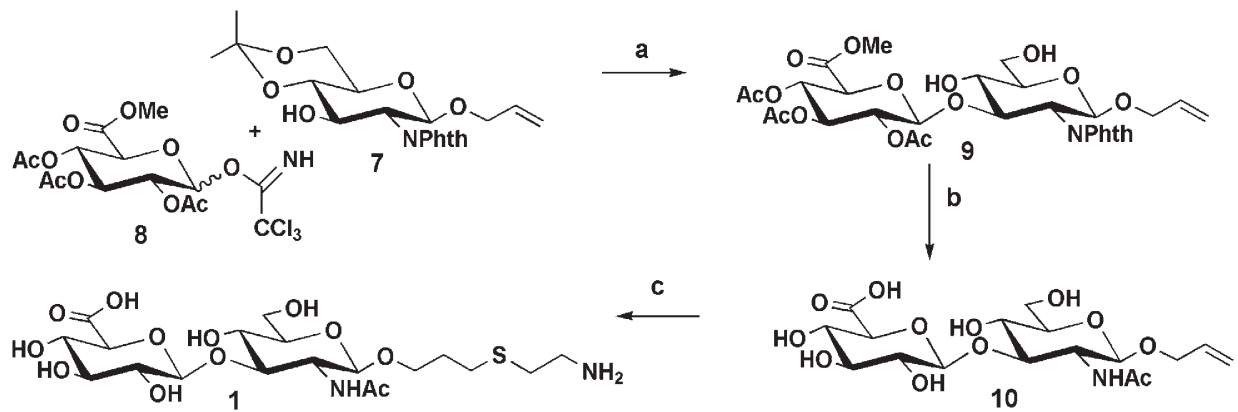

Scheme 1 Reagents and conditions: a, (i) 7, 8 (2 equiv.), TMSOTf, $\mathrm{CH}_{2} \mathrm{Cl}_{2}, 30$ min, $0{ }^{\circ} \mathrm{C} / 2 \mathrm{~h}, \mathrm{rt}$; (ii) water, $\mathrm{TFA}, \mathrm{CH}_{2} \mathrm{Cl}{ }_{2}, \mathrm{overnight}, 31 \%$ over two reaction steps; b, (i) $3 \mathrm{M}$ aq. $\mathrm{NaOH}, 5: 1 \mathrm{MeOH}$-water, $3 \mathrm{~h}$; (ii) $1: 21$,2-diaminoethane- $n$-butanol, overnight, $90^{\circ} \mathrm{C}$; (iii) acetic anhydride, MeOH, $3 \mathrm{~h}$, $0{ }^{\circ} \mathrm{C}, 52 \%$ over three reaction steps; c, cysteamine hydrochloride, UV-light, water, $2 \mathrm{~h}, 76 \%$. 

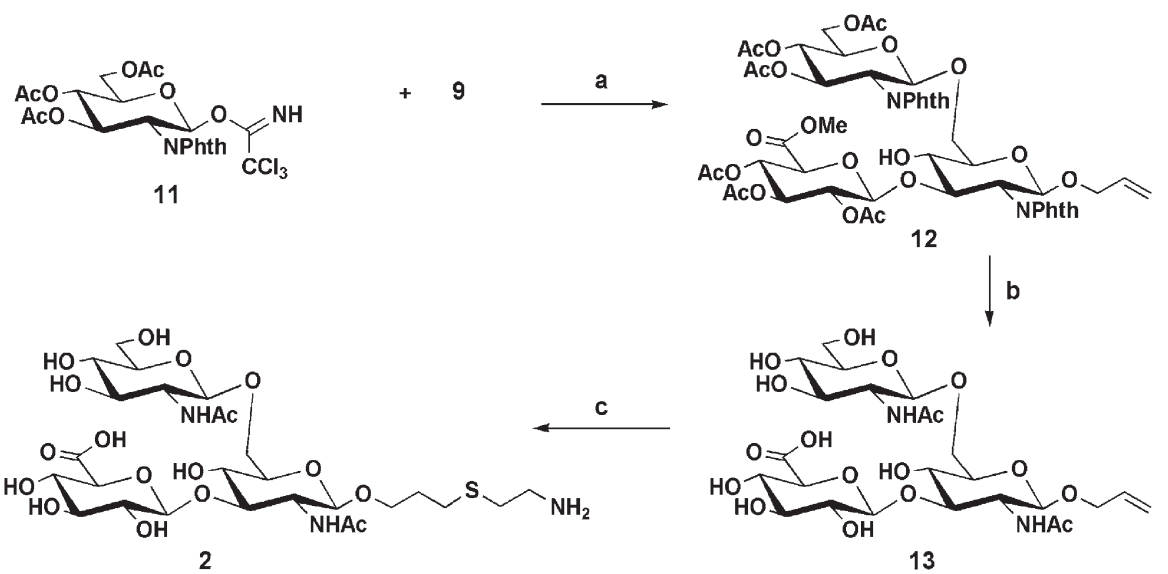

Scheme 2 Reagents and conditions: a, 9, 11 (1.5 equiv.), TMSOTf, $\mathrm{CH}_{2} \mathrm{Cl}_{2}, 1 \mathrm{~h}, 0{ }^{\circ} \mathrm{C}, 86 \%$; b, (i) $3 \mathrm{M}$ aq. $\mathrm{NaOH}, 5: 1 \mathrm{MeOH}-$ water, 3 h; (ii) $1: 2$ 1,2-diaminoethane- $n$-butanol, overnight, $90^{\circ} \mathrm{C}$; (iii) acetic anhydride, $\mathrm{MeOH}, 3 \mathrm{~h}, 0{ }^{\circ} \mathrm{C}, 42 \%$ over three reaction steps; c, cysteamine hydrochloride, UV-light, water, 2 h, $48 \%$.
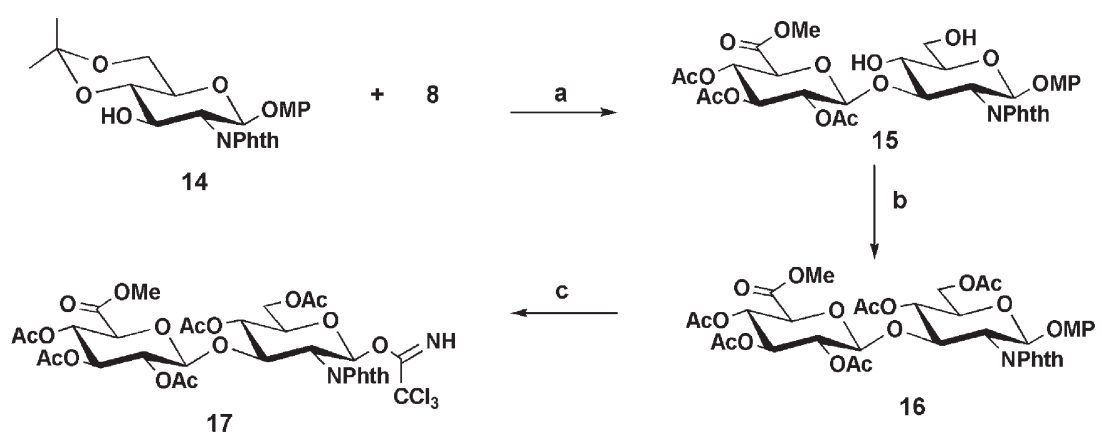

Scheme 3 Reagents and conditions: a, (i) 14, 8 (1.5 equiv.), TMSOTf, $\mathrm{CH}_{2} \mathrm{Cl}_{2}, 45 \mathrm{~min}, 0{ }^{\circ} \mathrm{C} / 30 \mathrm{~min}, \mathrm{rt}$; (ii) water, TFA, $\mathrm{CH}{ }_{2} \mathrm{Cl}{ }_{2}, 1 \mathrm{~h}, 71 \%$ over two reaction steps; b, $1: 1$ pyridine-acetic anhydride, $\mathrm{CH}_{2} \mathrm{Cl}_{2}$, overnight, quantitative; c, (i) CAN, $1: 1: 1$ toluene-acetonitrile-water, 45 min; (ii) trichloroacetonitrile, DBU, $\mathrm{CH}_{2} \mathrm{Cl}_{2}$, overnight, $63 \%$ over two reaction steps. $\mathrm{MP}=\mathrm{C}_{6} \mathrm{H}_{4} \mathrm{OCH}_{3}$.

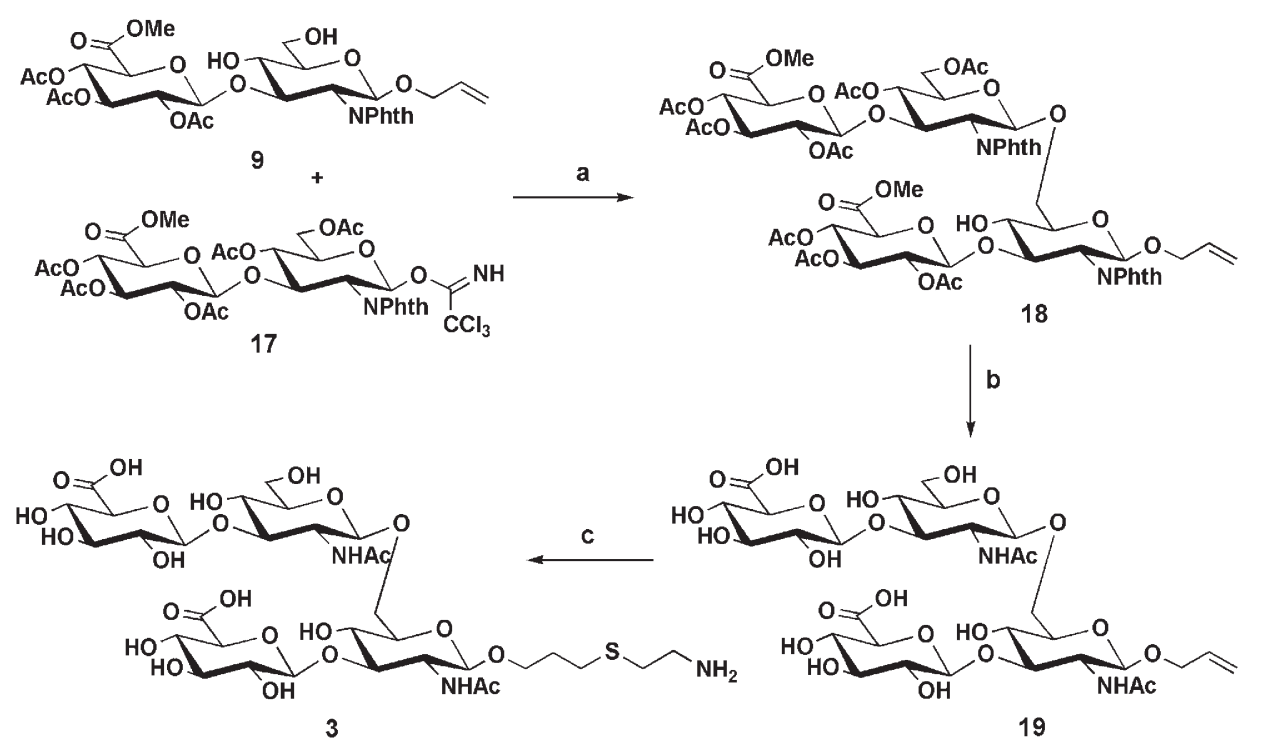

Scheme 4 Reagents and conditions: a, 9, 17 (1.5 equiv.), TMSOTf, $\mathrm{CH}_{2} \mathrm{Cl}_{2}, 1 \mathrm{~h}, 0{ }^{\circ} \mathrm{C}, 90 \%$; b, (i) $3 \mathrm{M}$ aq. $\mathrm{NaOH}, 5: 1 \mathrm{MeOH}-$ water, 3 h; (ii) $1: 2$ 1,2-diaminoethane- $n$-butanol, overnight, $90^{\circ} \mathrm{C}$; (iii) acetic anhydride, $\mathrm{MeOH}, 3 \mathrm{~h}, 0{ }^{\circ} \mathrm{C}, 42 \%$ over three reaction steps; $\mathbf{c}$, cysteamine hydrochloride, UV-light, water, 2 h, $56 \%$.

\section{Synthesis of oligosaccharide analogues 4-6 containing $\beta$-D- Glcp $6 S$ instead of $\beta$-D-GlcpA moieties}

In the synthetic routes to target oligosaccharides $\mathbf{4 , 5}$, and $\mathbf{6}$ (Fig. 1), the disaccharide 4-methoxyphenyl(6-O-levulinoyl-2,3,4tri- $O$ - $p$-toluoyl- $\beta$-D-glucopyranosyl)- $(1 \rightarrow 3)-4-O$-acetyl-6- $O$ tert-butyldiphenylsilyl-2-deoxy-2-phthalimido- $\beta$-D-galactopyranoside 24 was used as a central building block, easy to transform into either a donor or an acceptor. The levulinoyl group at HO6 of the $\beta$-D-glucose residue can be selectively removed, and subsequently sulfated to prepare the desired mimic structures. Coupling of donor 6-O-levulinoyl-2,3,4-tri- $O-p$-toluoyl- $\alpha$ -
D-glucopyranosyl trichloroacetimidate $\mathbf{2 0}^{15}$ with acceptor 4-methoxyphenyl 4,6-O-benzylidene-2-deoxy-2-phthalimido$\beta$-D-glucopyranoside $\mathbf{2 1}{ }^{22}$ using trimethylsilyl triflate as a promoter, followed by the removal of the benzylidene group under acidic conditions, ${ }^{15}$ afforded disaccharide 22 (78\%) (Scheme 5). It should be noted that the use of an isopropylidene instead of a benzylidene protecting group gives rise to much lower yields. A tert-butyldiphenylsilyl group was selectively introduced at HO6 of 22 using tert-butyldiphenylsilyl chloride in the presence of $2: 1$ pyridine-triethylamine and a catalytic amount of 4-dimethylaminopyridine, to give $\mathbf{2 3}$ in 
91\% yield. ${ }^{23}$ At this stage, the $\beta$-D-glucosamine residue was transformed into the desired $\beta$-D-galactosamine residue by epimerization of the $\mathrm{HO} 4$ function. ${ }^{16}$ To this end, 23 was treated with trifluoromethanesulfonic anhydride in the presence of pyridine and a catalytic amount of 4-dimethylaminopyridine. The $\mathrm{S}_{\mathrm{N}} 2$ displacement of the introduced triflate group at $\mathrm{O} 4$ using tetrabutylammoniun acetate in DMF resulted in the desired 4- $O$-acetylated disaccharide $24(70 \%)$.

For the synthesis of disaccharide 4, the anomeric 4-methoxyphenyl group was oxidatively removed using ammonium cerium(IV) nitrate, and subsequent imidation of the hemiacetal yielded disaccharide donor 25 (61\%). Coupling of 25 with 5-azidopentanol, using trimethylsilyl triflate as a promoter resulted in the azido-spacer-containing disaccharide 26 (85\%; Scheme 6). Removal of the tert-butyldiphenylsilyl group, under neutral conditions, using tetrabutylammonium fluoride $(\rightarrow \mathbf{2 7}$, $91 \%),{ }^{24}$ followed by acetylation under conventional conditions gave disaccharide $\mathbf{2 8}(83 \%)$. After selective removal of the levulinoyl group using hydrazine acetate $(\rightarrow \mathbf{2 9}, 91 \%),{ }^{25,26}$ sulfation of the liberated HO6' group was accomplished using the sulfur trioxide trimethylamine complex $(\rightarrow \mathbf{3 0}, 50 \%) .{ }^{27}$ Dephthaloylation/deacylation with ethanolic 33\% methylamine (7 days), and $\mathrm{N}$-acetylation with acetic anhydride in methanol at $0{ }^{\circ} \mathrm{C},{ }^{16}$ yielded azido-spacer-containing disaccharide 31 (76\%). Finally, catalytic hydrogenation of the azido group of $\mathbf{3 1}$ using $10 \%$ palladium on charcoal and sodium borohydride ${ }^{11}$ gave the amino-spacer-containing disaccharide 4 (71\%).

For the synthesis of trisaccharide 5, disaccharide acceptor 27 was coupled with donor 3,4,6-tri- $O$-acetyl-2-deoxy-2phthalimido- $\beta$-D-galactopyranosyl trichloroacetimidate $\mathbf{3 2},{ }^{28}$ using trimethylsilyl triflate as a promoter, to yield trisaccharide $33(58 \%$; Scheme 7). As the 6-O-acetylated variant of $\mathbf{2 7}$ is the main side product isolated from this condensation reaction, this moderate yield can be explained by the loss of acceptor during coupling due to in situ $O$-acetyl migration from $\mathrm{HO} 4$ to HO6. Delevulinoylation of $\mathbf{3 3}$ using hydrazine acetate $(\rightarrow \mathbf{3 4}$, $74 \%$ ), followed by sulfation of the generated HO6" group yielded sulfated trisaccharide 35 in $82 \%$ overall yield. Finally, dephthaloylation/deacylation followed by $\mathrm{N}$-acetylation afforded the azido-spacer-containing trisaccharide 36 (74\%), of which the azido group was selectively hydrogenated to give the amino-spacer-containing trisaccharide $\mathbf{5}(89 \%)$.

In order to synthesize tetrasaccharide $\mathbf{6}$, disaccharide donor 38 was prepared from disaccharide building block $\mathbf{2 4}$ in a two-step reaction sequence (Scheme 8). Removal of the tertbutyldiphenylsilyl group of $\mathbf{2 4}$ using tetrabutylammonium fluoride was directly followed by conventional acetylation of the generated HO6 function ( $\rightarrow 37,84 \%)$. Oxidative removal of the anomeric 4-methoxyphenyl group with ammonium cerium(IV) nitrate, followed by imidation gave $\mathbf{3 8}$ in $58 \%$ overall yield. Condensation of $\mathbf{2 7}$ with $\mathbf{3 8}$ in the presence of trimethylsilyl triflate afforded tetrasaccharide $\mathbf{3 9}(70 \%)$. Treatment of $\mathbf{3 9}$ with hydrazine acetate $(\rightarrow \mathbf{4 0}, 75 \%)$, sulfation of the two generated HO6 groups with the sulfur trioxide trimethylamine complex $(\rightarrow \mathbf{4 1}, 77 \%)$, and dephthaloylation/deacylation followed by $\mathrm{N}$-acetylation gave the disulfated azido-spacer-containing tetrasaccharide $42(62 \%)$. Finally, catalytic hydrogenation of the azido group yielded the amino-spacer-containing tetrasaccharide $6(71 \%)$.

\section{Preparation of neoglycoconjugates BSA-1-BSA-6}

Compounds 1-6 were conjugated to pre-treated bovine serum albumin $(\mathrm{BSA})^{11}$ using diethyl squarate as a linker. Reaction of the amine functions of 1-6 with diethyl squarate was performed in ethanol-50 $\mathrm{mM}$ sodium phosphate
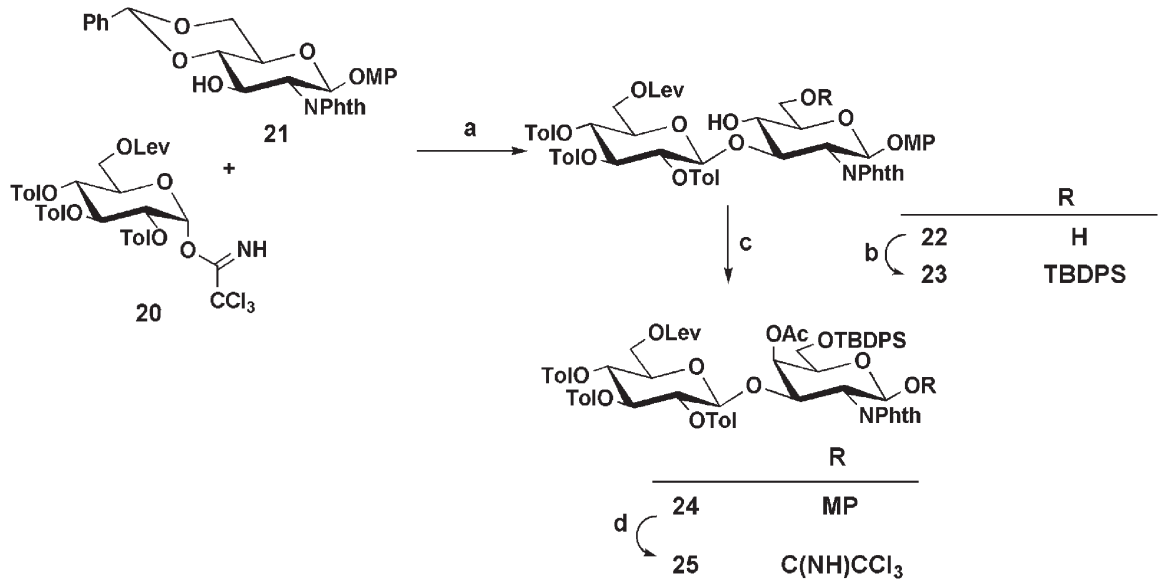

Scheme 5 Reagents and conditions: a, (i) 21, 20 (1.5 equiv.), TMSOTf, $\mathrm{CH}_{2} \mathrm{Cl}_{2}, 30$ min; (ii) water, TFA, $\mathrm{CH}_{2} \mathrm{Cl}_{2}, 78 \%$ over two reaction steps; b, 2 : 1 pyridine- $\mathrm{Et}_{3} \mathrm{~N}$, catalytic DMAP, $\mathrm{CH}_{2} \mathrm{Cl}_{2}$, TBDPSCl, overnight, $91 \%$; c, (i) trifluoromethanesulfonic anhydride, pyridine, catalytic DMAP, $\mathrm{CH}_{2} \mathrm{Cl}_{2}, 30$ min, $0{ }^{\circ} \mathrm{C} / 5 \mathrm{~h}$, rt; (ii) TBAA, DMF, $2 \mathrm{~h}, 70 \%$ over two reaction steps; d, (i) CAN, $1: 1: 1$ toluene-acetonitrile-water, 2 h; (ii) trichloroacetonitrile, $\mathrm{DBU}$, $\mathrm{CH}_{2} \mathrm{Cl}_{2}$, $3 \mathrm{~h}, 61 \%$ over two reaction steps. $\mathrm{Lev}=\mathrm{COCH}_{2} \mathrm{CH}_{2} \mathrm{COCH}_{3} ; \mathrm{MP}=\mathrm{C}_{6} \mathrm{H}_{4} \mathrm{OCH}_{3} ; \mathrm{Ph}=\mathrm{C}_{6} \mathrm{H}_{5} ; \mathrm{Tol}=\mathrm{COC}_{6} \mathrm{H}_{4} \mathrm{CH}_{3} ; \mathrm{TBDPS}=\left(\mathrm{CH}_{3}\right)_{3} \mathrm{CSi}_{2}\left(\mathrm{C}_{6} \mathrm{H}_{5}\right)_{2}$.

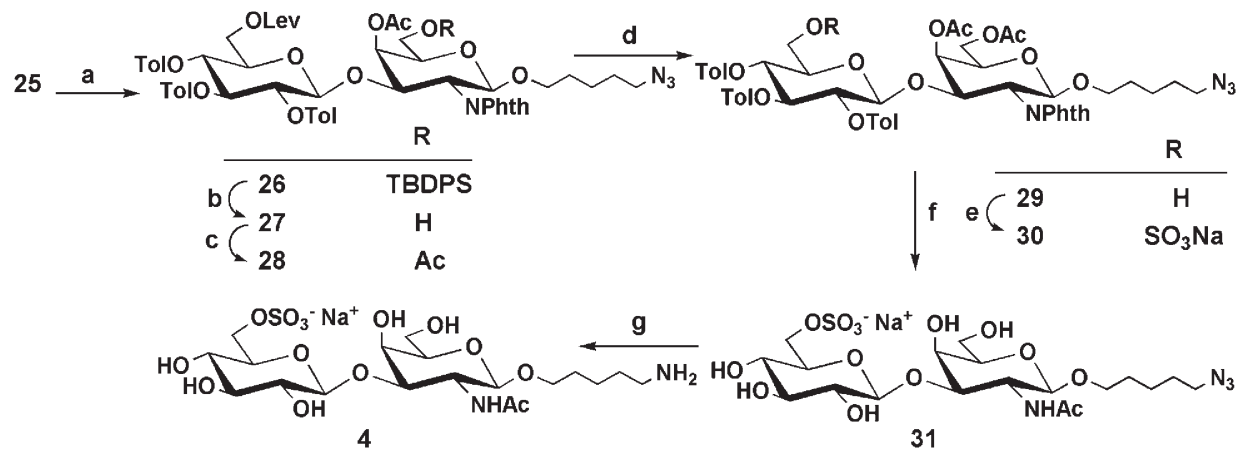

Scheme 6 Reagents and conditions: a, 25, 5-azidopentanol (2 equiv.), TMSOTf, $\mathrm{CH}_{2} \mathrm{Cl}_{2}, 30 \mathrm{~min}, 85 \%$; b, $1 \mathrm{M} \mathrm{TBAF}$ in THF, HOAc, pH 7, 2 h, $0{ }^{\circ} \mathrm{C} / 4 \mathrm{~h}, \mathrm{rt}, 91 \%$; c, $1: 1$ pyridine-acetic anhydride, overnight, $83 \%$; d, $\mathrm{NH}_{2} \mathrm{NH}_{2} \cdot \mathrm{HOAc}, \mathrm{EtOH}$, toluene, $2 \mathrm{~h}, 91 \%$; e, $\mathrm{SO} \cdot \mathrm{NMe}_{3}, \mathrm{DMF}, 48 \mathrm{~h}, 50{ }^{\circ} \mathrm{C}$, $50 \%$; f, (i) $33 \% \mathrm{NH}_{2} \mathrm{Me}$ in EtOH, $7 \mathrm{~d}$; (ii) acetic anhydride, $\mathrm{MeOH}, 3 \mathrm{~h}, 0{ }^{\circ} \mathrm{C}, 76 \%$ over two reaction steps; g, $0.05 \mathrm{M}$ aq. $\mathrm{NaOH}, 10 \% \mathrm{Pd}-\mathrm{C}, \mathrm{NaBH}$, water, $45 \mathrm{~min}, 71 \%$. Lev $\left.=\mathrm{COCH}_{2} \mathrm{CH}_{2} \mathrm{COCH}_{3} ; \mathrm{Tol}=\mathrm{COC}_{6} \mathrm{H}_{4} \mathrm{CH}_{3} ; \mathrm{TBDPS}=\left(\mathrm{CH}_{3}\right)_{3} \mathrm{CSi}_{(\mathrm{C}} \mathrm{C}_{5}\right)_{2}$. 

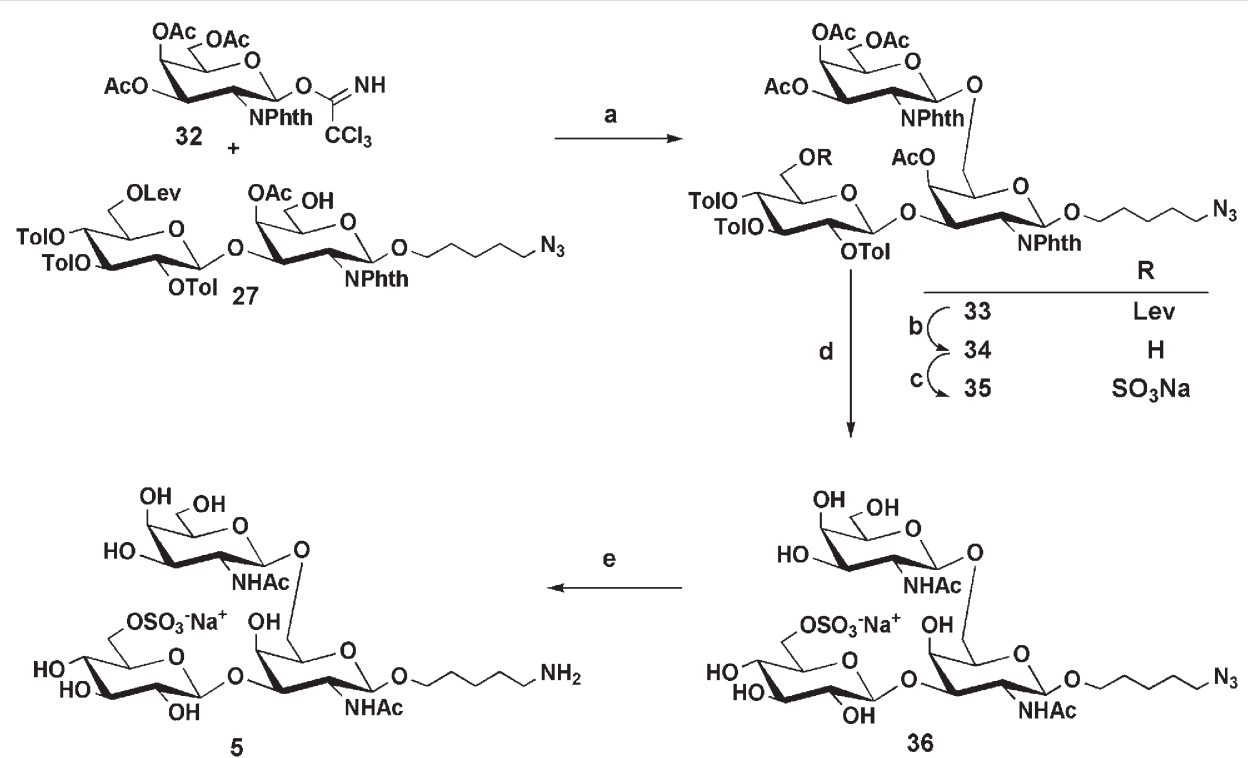

Scheme 7 Reagents and conditions: a, 27, 32 (1.5 equiv.), TMSOTf, $\mathrm{CH}_{2} \mathrm{Cl}_{2}, 15 \mathrm{~min}, 0{ }^{\circ} \mathrm{C}, 58 \%$; $\mathbf{b}, \mathrm{NH}_{2} \mathrm{NH}_{2} \cdot \mathrm{HOAc}$, EtOH, toluene, $2 \mathrm{~h}, 74 \%$; c, $\mathrm{SO}_{3} \cdot \mathrm{NMe}_{3}, \mathrm{DMF}, 48 \mathrm{~h}, 50^{\circ} \mathrm{C}, 82 \%$; d, (i) $33 \% \mathrm{NH}_{2} \mathrm{Me}$ in EtOH, $7 \mathrm{~d}$; (ii) acetic anhydride, $\mathrm{MeOH}, 3 \mathrm{~h}, 0{ }^{\circ} \mathrm{C}, 74 \%$ over two reaction steps; e, $0.05 \mathrm{M}$ aq. $\mathrm{NaOH}, 10 \% \mathrm{Pd}-\mathrm{C}, \mathrm{NaBH}_{4}$, water, $1 \mathrm{~h}, 89 \%$. $\mathrm{Lev}=\mathrm{COCH}_{2} \mathrm{CH}_{2} \mathrm{COCH}_{3} ; \mathrm{Tol}=\mathrm{COC}_{6} \mathrm{H}_{4} \mathrm{CH}_{3}$.

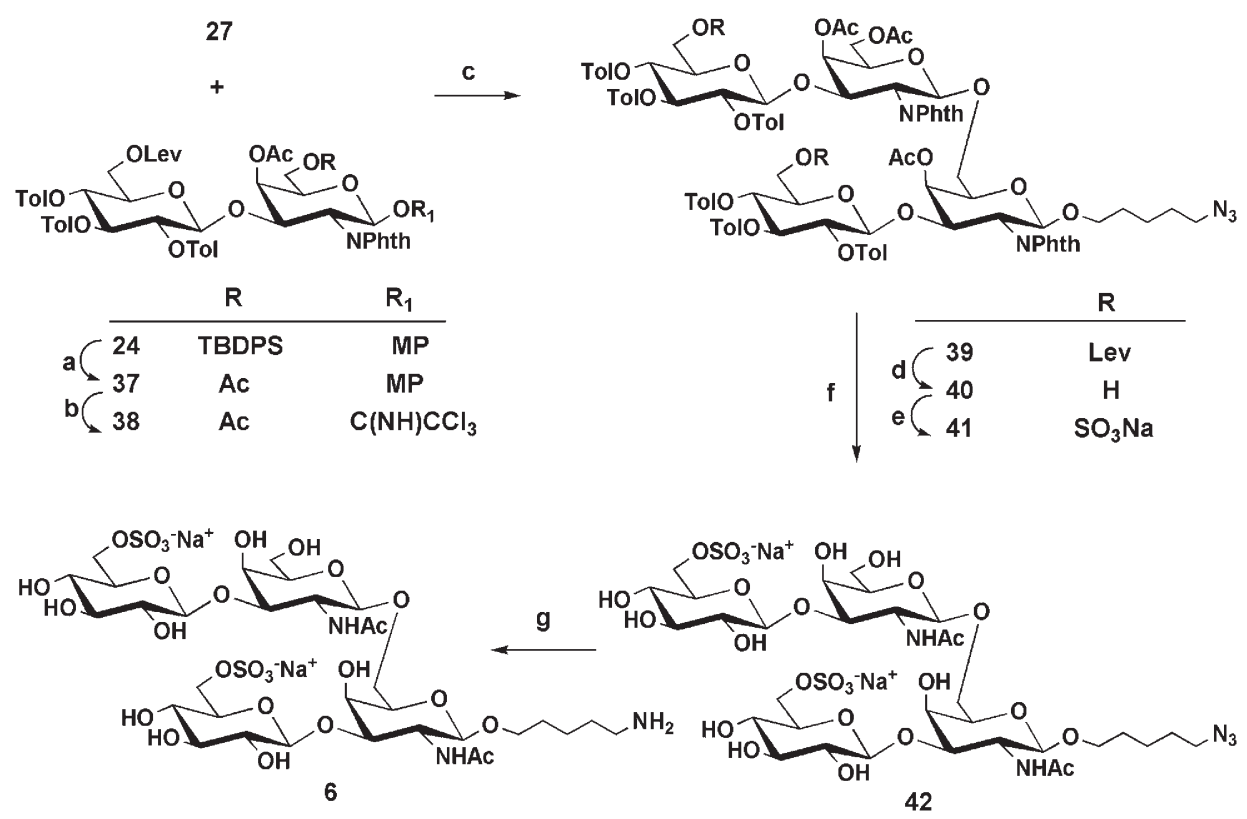

Scheme 8 Reagents and conditions: a, (i) $1 \mathrm{M} \mathrm{TBAF}$ in THF, HOAc, pH 7, $1 \mathrm{~h}, 0{ }^{\circ} \mathrm{C} /$ overnight, rt; (ii) $1: 1$ pyridine-acetic anhydride, overnight, $84 \%$ over two reaction steps; b, CAN, 1:1:1 toluene-acetonitrile-water, 2 h; (ii) trichloroacetonitrile, $\mathrm{DBU}^{\mathrm{C}} \mathrm{CH}_{2} \mathrm{Cl}_{2}, 16 \mathrm{~h}, 58 \%$ over two reaction steps; c, 27, 38 (1.5 equiv.), TMSOTf, $\mathrm{CH}_{2} \mathrm{Cl}_{2}, 15 \mathrm{~min}, 70 \%$; d, $\mathrm{NH}_{2} \mathrm{NH}_{2} \cdot \mathrm{HOAc}$, EtOH, toluene, $2 \mathrm{~h}, 75 \%$; e, $\mathrm{SO}_{3} \cdot \mathrm{NMe}_{3}, \mathrm{DMF}, 48 \mathrm{~h}, 50{ }^{\circ} \mathrm{C}, 77 \%$; f, (i) $33 \%$ $\mathrm{NH}_{2} \mathrm{Me}$ in EtOH, $7 \mathrm{~d}$; (ii) acetic anhydride, $\mathrm{MeOH}, 3 \mathrm{~h}$ at $0{ }^{\circ} \mathrm{C}, 62 \%$ over two reaction steps; $\mathbf{g}, 0.05 \mathrm{M}$ aq. $\mathrm{NaOH}, 10 \% \mathrm{Pd}-\mathrm{C}, \mathrm{NaBH}$, water, $1 \mathrm{~h}, 71 \%$. $\mathrm{Lev}=\mathrm{COCH}_{2} \mathrm{CH}_{2} \mathrm{COCH}_{3} ; \mathrm{MP}=\mathrm{C}_{6} \mathrm{H}_{4} \mathrm{OCH}_{3} ; \mathrm{Tol}=\mathrm{COC}_{6} \mathrm{H}_{4} \mathrm{CH}_{3} ; \mathrm{TBDPS}=\left(\mathrm{CH}_{3}\right)_{3} \mathrm{CSi}\left(\mathrm{C}_{6} \mathrm{H}_{5}\right)_{2}$.

buffer ( $\mathrm{pH} 7.2) .^{29,30}$ The obtained squarate-linker-containing disaccharides 43 and $\mathbf{4 6}$ were purified by solid phase extraction on a C-18 Extract-Clean ${ }^{\mathrm{TM}}$ column. However, purification of the larger saccharides $(\mathbf{4 4}, \mathbf{4 5}, \mathbf{4 7}$, and $\mathbf{4 8})$ needed another protocol; here, column chromatography on silica gel $(7.5: 1.5: 1.0$ EtOAc$\mathrm{MeOH}$-water) was used. The isolated squarate-linker-containing saccharides $\mathbf{4 3 - 4 8}$ were directly-coupled to BSA in $0.1 \mathrm{M}$ sodium bicarbonate buffer at pH 9.0 (Fig. 2).

As already observed in previous studies, ${ }^{11,31}$ the conjugation yield of an oligosaccharide to BSA decreases as its size increases. The average number of carbohydrate fragments incorporated in BSA was measured using MALDI-TOF MS analysis by determination of the center of the distribution of the singlecharged molecular ion (Fig. 3). The neoglycoconjugates BSA-1-BSA-6 have been applied to a panel of MAbs against Schistosoma mansoni antigens in immunoreactivity studies, using ELISA and surface plasmon resonance detection. The results of this work will be published elsewhere.

\section{Experimental}

\section{General procedures}

All chemicals were of reagent grade, and were used without further purification. Reactions were monitored by TLC on Silica Gel $60 \mathrm{~F}_{254}$ (Merck); after examination under UV-light, compounds were visualized by heating with $10 \%$ methanolic $\mathrm{H}_{2} \mathrm{SO}_{4}$, orcinol $\left(2 \mathrm{mg} \mathrm{cm}^{-3}\right)$ in $20 \%$ methanolic $\mathrm{H}_{2} \mathrm{SO}_{4}$, or ninhydrin $\left(1.5 \mathrm{mg} \mathrm{cm}^{-3}\right)$ in $38: 1.75: 0.251-\mathrm{BuOH}-\mathrm{H}_{2} \mathrm{O}-\mathrm{HOAc}$. In the work-up procedures of reaction mixtures, organic solutions were washed with appropriate amounts of the indicated aqueous solutions, then dried with $\mathrm{MgSO}_{4}$, and concentrated under reduced pressure at $30-50{ }^{\circ} \mathrm{C}$ on a water bath. Column chromatography was performed on Silica Gel 60 (Merck, 0.040-0.063 mm). Optical rotations were measured with a Perkin-Elmer 241 polarimeter, using a $10 \mathrm{~cm}, 1 \mathrm{~cm}^{3}$ cell. ${ }^{1} \mathrm{H}$ NMR spectra were recorded at $300 \mathrm{~K}$ with a Bruker AC 300 (300 MHz) or a Bruker AMX $500(500 \mathrm{MHz})$ spectrometer; 

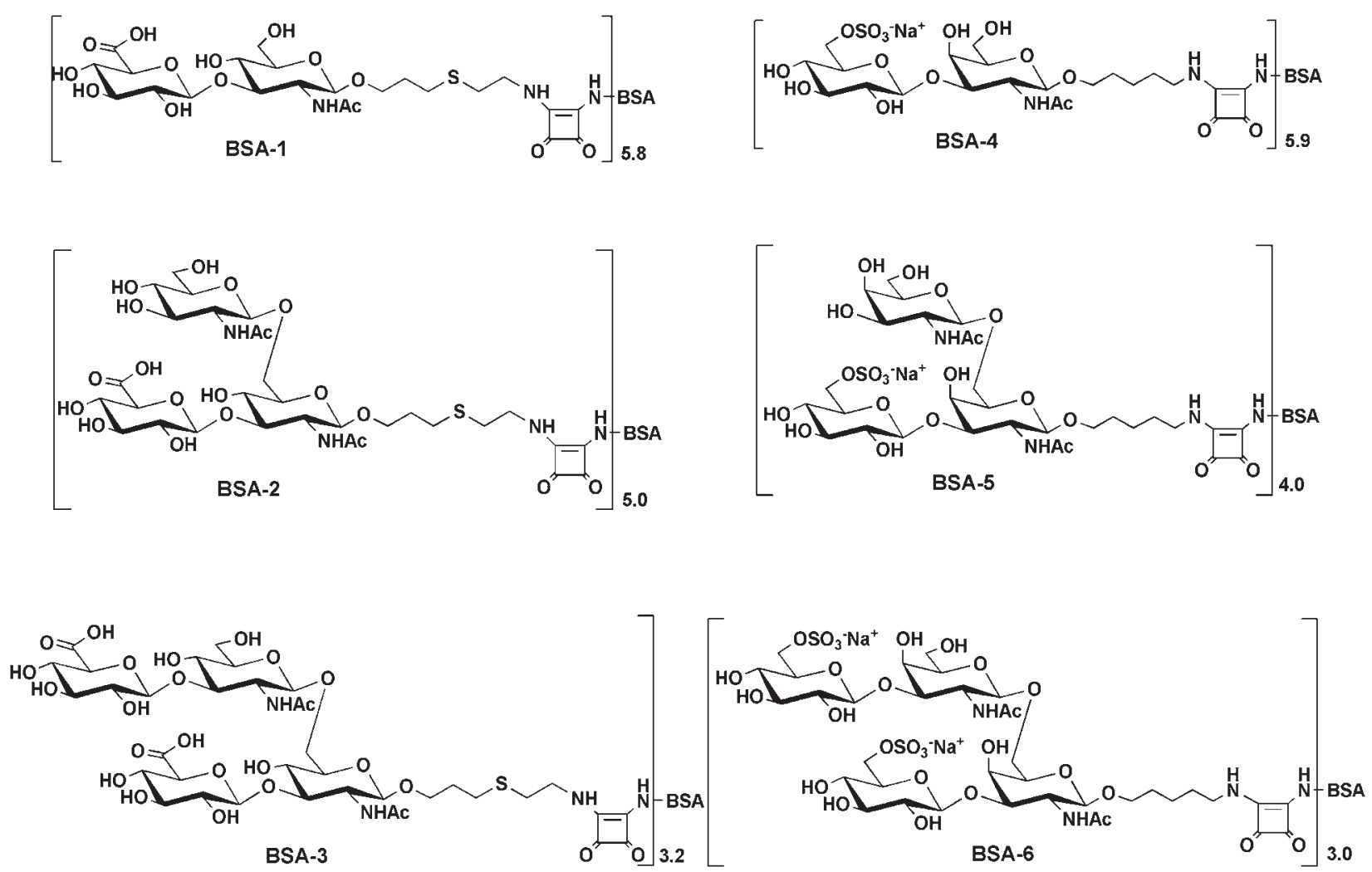

Fig. 2 Neoglycoconjugates BSA-1-BSA-6.

(a)

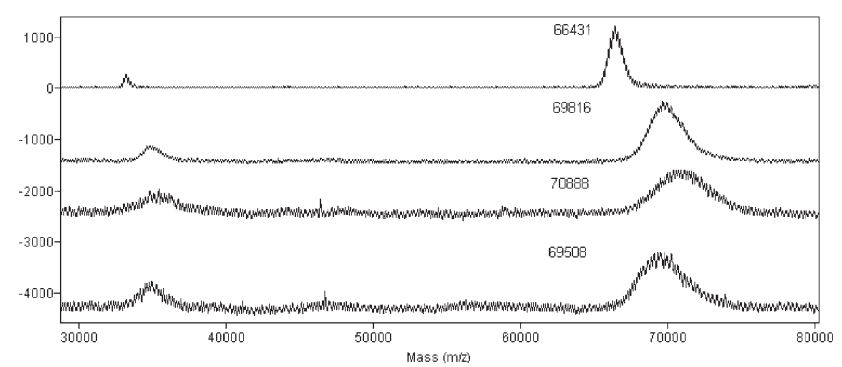

(b)

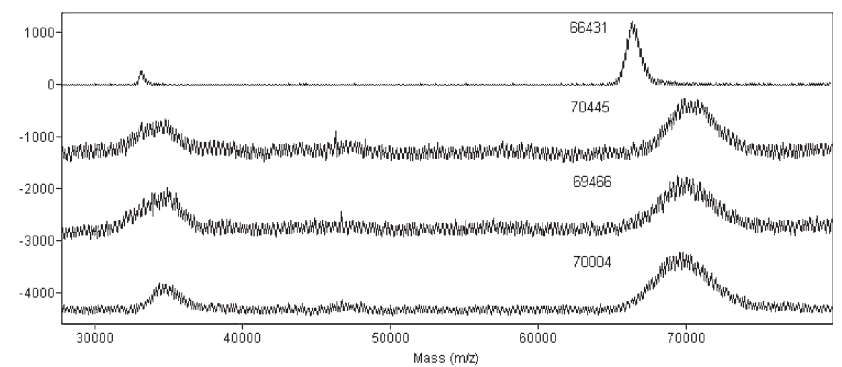

Fig. 3 MALDI-TOF MS spectra: (a) BSA (top), BSA-1 $(n=5.8$, c.e. $58 \%)$, $\mathbf{B S A}-2(n=5.0$, c.e. $50 \%)$, and $\mathbf{B S A}-3(n=3.2$, c.e. $32 \%)$; (b) BSA (top), BSA-4 $(n=5.9$, c.e. $59 \%)$, BSA-5 $(n=4.0$, c.e. $40 \%)$, and BSA-6 $(n=3.0$, c.e. $30 \%) . n=$ oligosaccharide loading; c.e. = coupling efficiency.

$\delta_{\mathrm{H}}$ values are given in ppm relative to the signal for internal $\mathrm{Me}_{4} \mathrm{Si}\left(\delta_{\mathrm{H}}=0, \mathrm{CDCl}_{3}\right)$ or internal acetone $\left(\delta_{\mathrm{H}}=2.22, \mathrm{D}_{2} \mathrm{O}\right) \cdot{ }^{13} \mathrm{C}$ NMR spectra (APT, $75.5 \mathrm{MHz}$ ) were recorded at $300 \mathrm{~K}$ with a Bruker AC 300 spectrometer; $\delta_{\mathrm{C}}$ values are given in ppm relative to the signal of $\mathrm{CDCl}_{3}\left(\delta_{\mathrm{C}}=77.1, \mathrm{CDCl}_{3}\right)$ or internal acetone $\left(\delta_{\mathrm{C}}=30.9, \mathrm{D}_{2} \mathrm{O}\right)$. Two-dimensional ${ }^{1} \mathrm{H}-{ }^{1} \mathrm{H}$ TOCSY (mixing times 7 and $100 \mathrm{~ms}$ ) and ${ }^{1} \mathrm{H}-{ }^{13} \mathrm{C}$ correlated HSQC spectra were recorded at $300 \mathrm{~K}$ with a Bruker AMX 500 spectrometer. Exact masses were measured by matrix-assisted laser desorption ionization time-of-flight mass spectrometry using a Voyager-
DE Pro (Applied Biosystems) instrument in the reflector mode at a resolution of 5000 FWHM. 2,4-Dihydroxybenzoic acid in $\mathrm{H}_{2} \mathrm{O}\left(5 \mathrm{mg} \mathrm{cm}^{-3}\right)$ was used as a matrix. A ladder of maltose oligosaccharides (G3-G13) was added as internal standard.

Allyl (methyl 2,3,4-tri- $O$-acetyl- $\beta$-D-glucopyranosyluronate)$(1 \rightarrow 3)$-2-deoxy-2-phthalimido- $\beta$-D-glucopyranoside 9

A solution of allyl 2-deoxy-4,6-O-isopropylidene-2phthalimido- $\beta$-D-glucopyranoside ${ }^{13}$ (7; $\left.0.55 \mathrm{~g}, 1.41 \mathrm{mmol}\right)$ and methyl 2,3,4-tri- $O$-acetyl- $\alpha, \beta$-D-glucopyranosyluronate trichloroacetimidate $^{14}(\mathbf{8} ; 1.35 \mathrm{~g}, 2.82 \mathrm{mmol})$ in dry $\mathrm{CH}_{2} \mathrm{Cl}_{2}$ $\left(20 \mathrm{~cm}^{3}\right)$, containing activated molecular sieves (4 $\left.\AA, 1 \mathrm{~g}\right)$, was stirred for $1 \mathrm{~h}$ at $\mathrm{rt}$, then TMSOTf $\left(69 \mathrm{~mm}^{3}, 0.35 \mathrm{mmol}\right)$ was added at $0{ }^{\circ} \mathrm{C}$. The mixture was stirred for $30 \mathrm{~min}$ at $0{ }^{\circ} \mathrm{C}$ and $2 \mathrm{~h}$ at rt, when TLC $\left(95: 5 \mathrm{CH}_{2} \mathrm{Cl}_{2}\right.$-acetone) showed the formation of a new product $\left(R_{\mathrm{f}}=0.56\right)$. After neutralization with dry pyridine and filtration, the solution was washed with $10 \%$ aq. $\mathrm{NaCl}$, dried, filtered, and concentrated. To a solution of the residue in $\mathrm{CH}_{2} \mathrm{Cl}_{2}\left(20 \mathrm{~cm}^{3}\right)$ and water $\left(0.1 \mathrm{~cm}^{3}\right)$ was added TFA $\left(1 \mathrm{~cm}^{3}\right)$. The mixture was stirred overnight, when TLC $(95: 5$ $\mathrm{CH}_{2} \mathrm{Cl}_{2}$-acetone) showed the removal of the isopropylidene group to be complete $\left(R_{\mathrm{f}}=0.24\right)$. Then, the mixture was washed with saturated aq. $\mathrm{NaHCO}_{3}$, dried, filtered, and concentrated. Column chromatography $\left(9: 1 \mathrm{CH}_{2} \mathrm{Cl}_{2}\right.$-acetone $)$ of the residue gave $9(0.3 \mathrm{~g}, 31 \%)$, isolated as a yellow foam; $[\alpha]_{\mathrm{D}}^{20}-16(c 0.5$ in $\left.\mathrm{CHCl}_{3}\right) ; \delta_{\mathrm{H}}\left(300 \mathrm{MHz} ; \mathrm{CDCl}_{3}\right) 1.93,1.99$, and 2.16 (each $3 \mathrm{H}$, $3 \times \mathrm{s}, 3 \times \mathrm{Ac}), 3.54(1 \mathrm{H}, \mathrm{m}, \mathrm{H}-5), 3.67(1 \mathrm{H}$, br t, H-4), 3.76 $\left(3 \mathrm{H}, \mathrm{s}, \mathrm{COOCH}_{3}\right), 3.96(1 \mathrm{H}, \mathrm{m}, \mathrm{H}-6), 4.01$ and 4.22 (each $1 \mathrm{H}$, $\left.2 \times \mathrm{m}, \mathrm{OCH}_{2} \mathrm{CH}=\mathrm{CH}_{2}\right), 4.05\left(1 \mathrm{H}, \mathrm{d}, J_{\mathrm{H}-4^{\prime} \mathrm{H}-5^{\prime}} 9.6 \mathrm{~Hz}, \mathrm{H}-5^{\prime}\right)$, $4.22\left(1 \mathrm{H}, \mathrm{dd}, J_{\mathrm{H}-1, \mathrm{H}-2} 8.5, J_{\mathrm{H}-2, \mathrm{H}-3} 10.8, \mathrm{H}-2\right), 4.46\left(1 \mathrm{H}, \mathrm{d}, J_{\mathrm{H}-1^{\prime}, \mathrm{H}-2^{\prime}}\right.$ 7.8, H-1'), $4.53\left(1 \mathrm{H}, \mathrm{dd}, J_{\mathrm{H}-3, \mathrm{H}-4} 8.1, \mathrm{H}-3\right), 4.87\left(1 \mathrm{H}, \mathrm{br} \mathrm{t}, \mathrm{H}-2^{\prime}\right)$, $5.05(1 \mathrm{H}, \mathrm{d}, \mathrm{H}-1), 5.64\left(1 \mathrm{H}, \mathrm{m}, \mathrm{OCH}_{2} \mathrm{CH}=\mathrm{CH}_{2}\right), 7.78$ and 7.86 (each $2 \mathrm{H}, 2 \times \mathrm{m}$, Phth); $\delta_{\mathrm{C}}\left(75.5 \mathrm{MHz} ; \mathrm{CDCl}_{3}\right) 20.4\left(\mathrm{COCH}_{3}\right)$, $53.2\left(\mathrm{COOCH}_{3}\right), 54.9(\mathrm{C}-2), 63.0(\mathrm{C}-6), 69.9\left(\mathrm{OCH}_{2} \mathrm{CH}=\mathrm{CH}_{2}\right)$, 68.7, 70.6, 71.0, 71.4, 71.9, 75.5, and 81.8 (C-3, C-4, C-5, C$2^{\prime}, \mathrm{C}-3^{\prime}, \mathrm{C}-4^{\prime}$, and $\left.\mathrm{C}-5^{\prime}\right), 97.4$ and $100.0\left(\mathrm{C}-1\right.$ and $\left.\mathrm{C}-1^{\prime}\right), 117.4$ $\left(\mathrm{OCH}_{2} \mathrm{CH}=\mathrm{CH}_{2}\right), 133.5\left(\mathrm{OCH}_{2} \mathrm{CH}=\mathrm{CH}_{2}\right), 123.6$ and 134.6 $\left[\mathrm{N}(\mathrm{CO})_{2} \mathrm{C}_{6} \mathrm{H}_{4}\right]$; high resolution MALDI-TOF MS, $\mathrm{m} / \mathrm{z}$ found $\mathrm{M}+\mathrm{Na} 688.185, \mathrm{C}_{30} \mathrm{H}_{35} \mathrm{NNaO}_{16}$ requires 688.185. 


\section{Allyl ( $\beta$-D-glucopyranosyluronic acid)-( $1 \rightarrow 3)$-2-acetamido-2-} deoxy- $\beta$-D-glucopyranoside 10

To a solution of $9(28 \mathrm{mg}, 42 \mu \mathrm{mol})$ in $5: 1 \mathrm{MeOH}-$ water $\left(3 \mathrm{~cm}^{3}\right)$ was added, at $0{ }^{\circ} \mathrm{C}, 3 \mathrm{M}$ aq. $\mathrm{NaOH}\left(1 \mathrm{~cm}^{3}\right)$. The mixture was stirred for $3 \mathrm{~h}$ at room temperature, neutralized with Dowex $50 \times 8 \mathrm{H}^{+}$resin, filtered, and concentrated. A solution of the residue in 2:1 $n$-butanol-1,2-diaminoethane $\left(6 \mathrm{~cm}^{3}\right)$ was stirred overnight at $90{ }^{\circ} \mathrm{C}$, then co-concentrated with toluene. To a solution of the residue in dry $\mathrm{MeOH}\left(5 \mathrm{~cm}^{3}\right)$ was added, at $0{ }^{\circ} \mathrm{C}$, acetic anhydride $\left(100 \mathrm{~mm}^{3}\right)$. The mixture was stirred for $3 \mathrm{~h}$, then concentrated. Size-exclusion chromatography (Bio-Gel $\left.\mathrm{P}-2,100 \mathrm{mM} \mathrm{NH}_{4} \mathrm{HCO}_{3}\right)$ gave $\mathbf{1 0}(9.4 \mathrm{mg}, 52 \%)$, isolated after lyophilization from water, as a white amorphous powder; $[\alpha]_{\mathrm{D}}^{20}-96$ ( $c 0.6$ in water); $\delta_{\mathrm{H}}\left(500 \mathrm{MHz} ; \mathrm{D}_{2} \mathrm{O} ; 2 \mathrm{D}\right.$ TOCSY and HSQC) 2.02 (3 H, s, NAc), 3.34 (1 H, br t, H-2'), 3.45 (1 H, m, H-5), 3.49 and 3.50 (each $1 \mathrm{H}, 2 \times$ br t, $\mathrm{H}-3^{\prime}$ and $\left.\mathrm{H}-4^{\prime}\right), 3.52\left(1 \mathrm{H}, \mathrm{dd}, J_{\mathrm{H}-3, \mathrm{H}-4}\right.$ $\left.8.2 \mathrm{~Hz}, J_{\mathrm{H}-4, \mathrm{H}-5} 9.8 \mathrm{~Hz}, \mathrm{H}-4\right), 3.72\left(1 \mathrm{H}, \mathrm{d}, J_{\mathrm{H}-4^{\prime}, \mathrm{H}-5^{\prime}} 9.3, \mathrm{H}-5^{\prime}\right), 3.73$ (1 H, br t, H-3), $3.76\left(1 \mathrm{H}, \mathrm{dd}, J_{\mathrm{H}-5, \mathrm{H}-6 \mathrm{~b}} 5.5, J_{\mathrm{H}-6 \mathrm{a}, \mathrm{H}-6 \mathrm{~b}} 12.4, \mathrm{H}-6 \mathrm{~b}\right)$, $3.84\left(1 \mathrm{H}, \mathrm{dd}, J_{\mathrm{H}-1, \mathrm{H}-2} 8.6, J_{\mathrm{H}-2, \mathrm{H}-3} 10.2, \mathrm{H}-2\right), 3.91\left(1 \mathrm{H}, \mathrm{dd}, J_{\mathrm{H}-5, \mathrm{H}-6 \mathrm{a}}\right.$ 2.2, $\mathrm{H}-6 \mathrm{a}), 4.16$ and 4.33 (each $1 \mathrm{H}, 2 \times \mathrm{m}, \mathrm{OCH}_{2} \mathrm{CH}=\mathrm{CH}_{2}$ ), 4.46 $\left(1 \mathrm{H}, \mathrm{d}, J_{\mathrm{H}-1^{\prime}, \mathrm{H}-2^{\prime}} 7.8, \mathrm{H}_{-1}{ }^{\prime}\right), 4.58(1 \mathrm{H}, \mathrm{d}, \mathrm{H}-1), 5.25$ and 5.29 (each $\left.1 \mathrm{H}, 2 \times \mathrm{m}, \mathrm{OCH}_{2} \mathrm{CH}=\mathrm{CH}_{2}\right), 5.90\left(1 \mathrm{H}, \mathrm{m}, \mathrm{OCH}_{2} \mathrm{CH}=\mathrm{CH}_{2}\right)$; $\delta_{\mathrm{C}}\left(125 \mathrm{MHz} ; \mathrm{D}_{2} \mathrm{O}\right) 22.9\left(\mathrm{NDCOCH}_{3}\right), 55.1(\mathrm{C}-2), 61.5(\mathrm{C}-6)$, $69.5(\mathrm{C}-4), 71.2\left(\mathrm{OCH}_{2} \mathrm{CH}=\mathrm{CH}_{2}\right), 72.4$ and $76.2\left(\mathrm{C}-3^{\prime}\right.$ and $\mathrm{C}-$ $\left.4^{\prime}\right), 73.5$ (C-2'), 76.3 (C-5), 76.5 (C-5'), 83.7 (C-3), 100.7 (C-1), $103.7\left(\mathrm{C}-1^{\prime}\right), 119.0\left(\mathrm{OCH}_{2} \mathrm{CH}=\mathrm{CH}_{2}\right), 134.1\left(\mathrm{OCH}_{2} \mathrm{CH}=\mathrm{CH}_{2}\right)$; high resolution MALDI-TOF MS, $\mathrm{m} / \mathrm{z}$ found $\mathrm{M}+\mathrm{H} 438.164$, $\mathrm{C}_{17} \mathrm{H}_{28} \mathrm{NO}_{12}$ requires 438.161 .

3-(2-Aminoethylthio)propyl ( $\beta$-D-glucopyranosyluronic acid)$(1 \rightarrow 3)$-2-acetamido-2-deoxy- $\beta$-D-glucopyranoside 1

A solution of $\mathbf{1 0}(5 \mathrm{mg}, 11.4 \mu \mathrm{mol})$ and cysteamine hydrochloride (7.1 mg, $62.7 \mu \mathrm{mol})$ in water $\left(1 \mathrm{~cm}^{3}\right)$ was irradiated for $2 \mathrm{~h}$ in a quartz vial, using a VL-50C Vilber Lourmat UV Lamp. The mixture was loaded directly on to a size-exclusion column (BioGel P-2, $100 \mathrm{mM} \mathrm{NH}_{4} \mathrm{HCO}_{3}$ ), yielding 1 (4.5 mg, 76\%), isolated after lyophilization from water as a white, amorphous powder; $[a]_{\mathrm{D}}^{20}-93(c 0.3$ in water $) ; \delta_{\mathrm{H}}\left(500 \mathrm{MHz} ; \mathrm{D}_{2} \mathrm{O} ; 2 \mathrm{D}\right.$ TOCSY and HSQC) $1.85\left[2 \mathrm{H}, \mathrm{m}, \mathrm{OCH}_{2} \mathrm{CH}_{2} \mathrm{CH}_{2} \mathrm{~S}\left(\mathrm{CH}_{2}\right)_{2} \mathrm{ND}_{2}\right], 2.03(3 \mathrm{H}$, s, NAc), $2.62\left[2 \mathrm{H}\right.$, br t, $\mathrm{O}\left(\mathrm{CH}_{2}\right)_{2} \mathrm{CH}_{2} \mathrm{~S}\left(\mathrm{CH}_{2}\right)_{2} \mathrm{ND}_{2}$ ], 2.84 and 3.23 [each $2 \mathrm{H}, 2 \times$ br t, $\mathrm{O}\left(\mathrm{CH}_{2}\right)_{3} \mathrm{~S}\left(\mathrm{CH}_{2}\right)_{2} \mathrm{ND}_{2}$ ], $3.33\left(1 \mathrm{H}, \mathrm{dd}, J_{\mathrm{H}-1^{\prime}, \mathrm{H}-2^{\prime}}\right.$ $\left.8.0 \mathrm{~Hz}, J_{\mathrm{H}-2^{\prime}, \mathrm{H}-3^{\prime}} 8.6 \mathrm{~Hz}, \mathrm{H}-2^{\prime}\right), 3.46(1 \mathrm{H}, \mathrm{m}, \mathrm{H}-5), 3.48(1 \mathrm{H}, \mathrm{br}$ t, H-4'), 3.50 (1 H, br t, H-3'), $3.52(1 \mathrm{H}$, br t, H-4), 3.69 and 3.98 [each $1 \mathrm{H}, 2 \times \mathrm{m}, \mathrm{OCH}_{2}\left(\mathrm{CH}_{2}\right)_{2} \mathrm{~S}\left(\mathrm{CH}_{2}\right)_{2} \mathrm{ND}_{2}$ ], $3.72(1 \mathrm{H}, \mathrm{d}$, $\left.J_{\mathrm{H}-4^{\prime}, \mathrm{H}-5^{\prime}} 9.6, \mathrm{H}-5^{\prime}\right), 3.74\left(1 \mathrm{H}\right.$, br t, H-3), $3.75\left(1 \mathrm{H}, \mathrm{dd}, J_{\mathrm{H}-5, \mathrm{H}-6 \mathrm{~b}}\right.$ $\left.5.7, J_{\mathrm{H}-6 \mathrm{a}, \mathrm{H}-6 \mathrm{~b}} 12.4, \mathrm{H}-6 \mathrm{~b}\right), 3.81\left(1 \mathrm{H}, \mathrm{dd}, J_{\mathrm{H}-1, \mathrm{H}-2} 8.5, J_{\mathrm{H}-2, \mathrm{H}-3} 10.4\right.$, $\mathrm{H}-2), 3.91\left(1 \mathrm{H}, \mathrm{dd}, J_{\mathrm{H}-5, \mathrm{H}-6 \mathrm{a}} 2.1, \mathrm{H}-6 \mathrm{a}\right), 4.47$ (1 H, d, H-1'), $4.52(1 \mathrm{H}, \mathrm{d}, \mathrm{H}-1) ; \delta_{\mathrm{C}}\left(125 \mathrm{MHz} ; \mathrm{D}_{2} \mathrm{O}\right) 23.0\left(\mathrm{NDCOCH}_{3}\right), 27.7$ $\left[\mathrm{O}\left(\mathrm{CH}_{2}\right)_{2} \mathrm{CH}_{2} \mathrm{~S}\left(\mathrm{CH}_{2}\right)_{2} \mathrm{ND}_{2}\right], 28.9$ and $39.1\left[\mathrm{O}\left(\mathrm{CH}_{2}\right)_{3} \mathrm{~S}\left(\mathrm{CH}_{2}\right)_{2} \mathrm{ND}_{2}\right]$, $29.3\left[\mathrm{OCH}_{2} \mathrm{CH}_{2} \mathrm{CH}_{2} \mathrm{~S}\left(\mathrm{CH}_{2}\right)_{2} \mathrm{ND}_{2}\right.$ ], $55.3(\mathrm{C}-2), 61.5(\mathrm{C}-6), 69.4$ $\left[\mathrm{OCH}_{2}\left(\mathrm{CH}_{2}\right)_{2} \mathrm{~S}\left(\mathrm{CH}_{2}\right)_{2} \mathrm{ND}_{2}\right], 69.5(\mathrm{C}-4), 72.4\left(\mathrm{C}-3^{\prime}\right), 73.5\left(\mathrm{C}-2^{\prime}\right)$, 76.1 (C-4'), 76.2 (C-5), 76.6 (C-5'), 83.3 (C-3), 101.9 (C-1), 103.6 $\left(\mathrm{C}-1^{\prime}\right)$; high resolution MALDI-TOF $\mathrm{MS}, \mathrm{m} / \mathrm{z}$ found $\mathrm{M}+\mathrm{Na}$ 537.163, $\mathrm{C}_{19} \mathrm{H}_{34} \mathrm{~N}_{2} \mathrm{NaSO}_{12}$ requires 537.173.

\section{Allyl (3,4,6-tri- $O$-acetyl-2-deoxy-2-phthalimido- \\ $\beta$-D-glucopyranosyl)-( $1 \rightarrow 6)-[($ methyl \\ $2,3,4$-tri- $O$-acetyl- $\beta$-D-glucopyranosyluronate)-(1 $\rightarrow 3) \mid$-2-deoxy- 2-phthalimido- $\beta$-D-glucopyranoside 12}

A solution of $\mathbf{9}(50 \mathrm{mg}, 75 \mu \mathrm{mol})$ and 3,4,6-tri- $O$-acetyl-2-deoxy2-phthalimido- $\beta$-D-glucopyranosyl trichloroacetimidate ${ }^{19}$ (11; $65 \mathrm{mg}, 112 \mu \mathrm{mol})$ in dry $\mathrm{CH}_{2} \mathrm{Cl}_{2}\left(2 \mathrm{~cm}^{3}\right)$, containing activated molecular sieves ( $4 \AA, 0.1 \mathrm{~g}$ ), was stirred for $1 \mathrm{~h}$ at room temperature, then TMSOTf $\left(1.5 \mathrm{~mm}^{3}, 7.5 \mu \mathrm{mol}\right)$ was added at $0{ }^{\circ} \mathrm{C}$. The mixture was stirred for $1 \mathrm{~h}$ at $0{ }^{\circ} \mathrm{C}$, when TLC $\left(9: 1 \mathrm{CH}_{2} \mathrm{Cl}_{2}\right.$-acetone) showed the formation of a new product $\left(R_{\mathrm{f}}=0.45\right)$. After neutralization with dry pyridine and filtration, the solution was washed with $10 \%$ aq. $\mathrm{NaCl}$, dried, filtered, and concentrated. Column chromatography ( $9: 1 \mathrm{CH}_{2} \mathrm{Cl}_{2}$-acetone) of the residue gave $12(70 \mathrm{mg}, 86 \%)$, isolated as a white solid; $[\alpha]_{\mathrm{D}}^{20}-7(c 0.2$ in $\left.\mathrm{CHCl}_{3}\right) ; \delta_{\mathrm{H}}\left(500 \mathrm{MHz} ; \mathrm{CDCl}_{3} ; 2 \mathrm{D}\right.$ TOCSY and HSQC) 1.51 , $1.80,1.87,1.93,1.96$, and 2.07 (each $3 \mathrm{H}, 6 \times \mathrm{s}, 6 \times \mathrm{Ac}$ ), 3.21 $\left(1 \mathrm{H}, \mathrm{dd}, J_{\mathrm{H}-3, \mathrm{H}-4} 9.8 \mathrm{~Hz}, J_{\mathrm{H}-4, \mathrm{H}-5} 8.3 \mathrm{~Hz}, \mathrm{H}-4\right), 3.43(1 \mathrm{H}, \mathrm{m}, \mathrm{H}-5)$, $3.65\left(1 \mathrm{H}, \mathrm{dd}, J_{\mathrm{H}-5, \mathrm{H}-6 \mathrm{~b}} 6.3, J_{\mathrm{H}-6 \mathrm{a}, \mathrm{H}-6 \mathrm{~b}} 11.0, \mathrm{H}-6 \mathrm{~b}\right), 3.67$ and 3.87 (each $\left.1 \mathrm{H}, 2 \times \mathrm{m}, \mathrm{OCH}_{2} \mathrm{CH}=\mathrm{CH}_{2}\right), 3.69\left(3 \mathrm{H}, \mathrm{s}, \mathrm{COOCH}_{3}\right)$, $3.83\left(1 \mathrm{H}, \mathrm{m}, \mathrm{H}-5^{\prime}\right), 3.88$ (1 H, d, $\left.J_{\mathrm{H}-4^{\prime \prime}, \mathrm{H}-5^{\prime \prime}} 9.9, \mathrm{H}-5^{\prime \prime}\right), 3.97$ (1 H, $\left.\mathrm{dd}, J_{\mathrm{H}-1, \mathrm{H}-2} 8.4, J_{\mathrm{H}-2, \mathrm{H}-3} 10.7, \mathrm{H}-2\right), 4.12\left(1 \mathrm{H}, \mathrm{dd}, J_{\mathrm{H}-5^{\prime}, \mathrm{H}-6 \mathrm{a}^{\prime}} 2.3\right.$, $\left.J_{\mathrm{H}-6 \mathrm{a}^{\prime}, \mathrm{H}-6 \mathrm{~b}^{\prime}} 12.2, \mathrm{H}-6 \mathrm{a}^{\prime}\right), 4.20\left(1 \mathrm{H}, \mathrm{dd}, J_{\mathrm{H}-5, \mathrm{H}-6 \mathrm{a}} 1.4, \mathrm{H}-6 \mathrm{a}\right), 4.22$ $\left(1 \mathrm{H}, \mathrm{d}, J_{\mathrm{H}-1^{\prime \prime}, \mathrm{H}-2^{\prime \prime}} 8.0, \mathrm{H}-1^{\prime \prime}\right), 4.28(1 \mathrm{H}$, br t, H-3), $4.29(1 \mathrm{H}, \mathrm{m}$, $\left.\mathrm{H}-6 \mathrm{~b}^{\prime}\right), 4.30\left(1 \mathrm{H}, \mathrm{dd}, J_{\mathrm{H}-1^{\prime}, \mathrm{H}-2^{\prime}} 8.6, J_{\mathrm{H}-2^{\prime}, \mathrm{H}-3^{\prime}} 10.9, \mathrm{H}-2^{\prime}\right), 4.63(1 \mathrm{H}$, dd, $\left.J_{\mathrm{H}-2^{\prime \prime}, \mathrm{H}-3^{\prime \prime}} 9.5, \mathrm{H}-2^{\prime \prime}\right), 4.78$ (1 H, d, H-1), 4.86 and 4.90 (each $\left.1 \mathrm{H}, 2 \times \mathrm{m}, \mathrm{OCH}_{2} \mathrm{CH}=\mathrm{CH}_{2}\right), 4.92(1 \mathrm{H}$, br t, H-3"), $5.00(1 \mathrm{H}$, br t, H-4"), $5.11\left(1 \mathrm{H}, \mathrm{dd}, J_{\mathrm{H}-3^{\prime}, \mathrm{H}-4^{\prime}} 9.2, J_{\mathrm{H}-4^{\prime}, \mathrm{H}-5^{\prime}} 10.1, \mathrm{H}-4^{\prime}\right), 5.38$ $\left(1 \mathrm{H}, \mathrm{d}, \mathrm{H}^{-1}{ }^{\prime}\right), 5.40\left(1 \mathrm{H}, \mathrm{m}, \mathrm{OCH}_{2} \mathrm{CH}=\mathrm{CH}_{2}\right), 5.76(1 \mathrm{H}, \mathrm{dd}$, $\left.\mathrm{H}-3^{\prime}\right), 7.69$ and 7.78 (each $\left.4 \mathrm{H}, 2 \times \mathrm{m}, 2 \times \mathrm{Phth}\right) ; \delta_{\mathrm{C}}(125 \mathrm{MHz}$; $\left.\mathrm{CDCl}_{3}\right) 18.8,19.3,19.4,19.5,19.6$, and $19.8\left(6 \times \mathrm{COCH}_{3}\right)$, $52.2\left(\mathrm{COOCH}_{3}\right), 53.7\left(\mathrm{C}-2^{\prime}\right), 53.8(\mathrm{C}-2), 61.0\left(\mathrm{C}-6^{\prime}\right), 67.6(\mathrm{C}-$ $\left.4^{\prime \prime}\right), 68.0\left(\mathrm{OCH}_{2} \mathrm{CH}=\mathrm{CH}_{2}\right), 68.1\left(\mathrm{C}-4^{\prime}\right), 68.5(\mathrm{C}-6), 68.6(\mathrm{C}-4)$, $69.7\left(\mathrm{C}-2^{\prime \prime}\right), 69.8\left(\mathrm{C}-3^{\prime}\right), 70.2\left(\mathrm{C}-5^{\prime \prime}\right), 70.8\left(\mathrm{C}-5^{\prime}\right), 70.9\left(\mathrm{C}-3^{\prime \prime}\right)$, 74.1 (C-5), 80.4 (C-3), 95.8 (C-1), 97.9 (C-1'), 98.7 (C-1"), 116.4 $\left(\mathrm{OCH}_{2} \mathrm{CH}=\mathrm{CH}_{2}\right), 132.4\left(\mathrm{OCH}_{2} \mathrm{CH}=\mathrm{CH}_{2}\right), 122.7$ and 133.4 $\left[\mathrm{N}(\mathrm{CO})_{2} \mathrm{C}_{6} \mathrm{H}_{4}\right.$ ]; high resolution MALDI-TOF MS, $\mathrm{m} / \mathrm{z}$ found $\mathrm{M}+\mathrm{Na}$ 1105.286, $\mathrm{C}_{50} \mathrm{H}_{54} \mathrm{~N}_{2} \mathrm{NaO}_{25}$ requires 1105.291.

\section{Allyl (2-acetamido-2-deoxy- $\beta$-D-glucopyranosyl)-(1 $\rightarrow 6)-$-[( $\beta$-D- glucopyranosyluronic acid)-(1 $\rightarrow 3) \mid-2$-acetamido-2-deoxy- $\beta$-D- glucopyranoside 13}

To a solution of $\mathbf{1 2}(26 \mathrm{mg}, 24 \mu \mathrm{mol})$ in $5: 1 \mathrm{MeOH}-$ water $\left(3 \mathrm{~cm}^{3}\right)$ was added, at $0{ }^{\circ} \mathrm{C}, 3 \mathrm{M}$ aq. $\mathrm{NaOH}\left(1 \mathrm{~cm}^{3}\right)$. The mixture was stirred for $3 \mathrm{~h}$ at room temperature, neutralized with

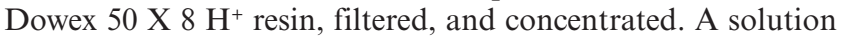
of the residue in 2:1 n-butanol-1,2-diaminoethane $\left(6 \mathrm{~cm}^{3}\right)$ was stirred overnight at $90{ }^{\circ} \mathrm{C}$, then co-concentrated with toluene. To a solution of the residue in dry $\mathrm{MeOH}\left(5 \mathrm{~cm}^{3}\right)$ was added, at $0{ }^{\circ} \mathrm{C}$, acetic anhydride $\left(100 \mathrm{~mm}^{3}\right)$. The mixture was stirred for $3 \mathrm{~h}$, then concentrated. Size-exclusion chromatography (Bio-Gel P-2, $\left.100 \mathrm{mM} \mathrm{NH}_{4} \mathrm{HCO}_{3}\right)$ gave 13 (6.5 mg, 42\%), isolated after lyophilization from water, as a white amorphous powder; $[\alpha]_{\mathrm{D}}^{20}-9$ ( $c 0.3$ in water); $\delta_{\mathrm{H}}\left(500 \mathrm{MHz} ; \mathrm{D}_{2} \mathrm{O} ; 2 \mathrm{D}\right.$ TOCSY and HSQC) 2.04 and 2.05 (each $3 \mathrm{H}, 2 \times \mathrm{s}, 2 \times \mathrm{NAc}), 3.34(1 \mathrm{H}$, br t, H-2"), 3.45 (1 H, br t, H-4'), $3.46\left(1 \mathrm{H}, \mathrm{m}, \mathrm{H}-5^{\prime}\right), 3.50(1 \mathrm{H}$, br t, H-3"), 3.51 (1 H, br t, H-4"), 3.52 (1 H, br t, H-4), 3.54 (1 H, m, H-5), 3.55 (1 H, br t, H-3'), $3.72\left(1 \mathrm{H}\right.$, br t, H-3), $3.73\left(1 \mathrm{H}, \mathrm{d}, \mathrm{H}-5^{\prime \prime}\right), 3.74$ $(1 \mathrm{H}$, br t, H-2' $), 3.75$ and 3.93 (each $\left.1 \mathrm{H}, 2 \times \mathrm{m}, 2 \times \mathrm{H}^{\prime} 6^{\prime}\right), 3.77$ and 4.20 (each $1 \mathrm{H}, 2 \times \mathrm{m}, 2 \times \mathrm{H}-6), 3.80(1 \mathrm{H}$, br t, H-2), 4.13 and 4.30 (each $\left.1 \mathrm{H}, 2 \times \mathrm{m}, \mathrm{OCH}_{2} \mathrm{CH}=\mathrm{CH}_{2}\right), 4.45\left(1 \mathrm{H}, \mathrm{d}, J_{\mathrm{H}-1^{\prime \prime}, \mathrm{H}-2^{\prime \prime}}\right.$ $\left.7.8, \mathrm{H}-1^{\prime \prime}\right), 4.52\left(1 \mathrm{H}, \mathrm{d}, J_{\mathrm{H}-1^{\prime}, \mathrm{H}-2^{\prime}} 8.4, \mathrm{H}^{\prime} 1^{\prime}\right), 4.56\left(1 \mathrm{H}, \mathrm{d}, J_{\mathrm{H}-1, \mathrm{H}-2}\right.$ 8.6, H-1), 5.26 and $5.30\left(\right.$ each $\left.1 \mathrm{H}, 2 \times \mathrm{m}, \mathrm{OCH}_{2} \mathrm{CH}=\mathrm{CH}_{2}\right)$, $5.88\left(1 \mathrm{H}, \mathrm{m}, \mathrm{OCH}_{2} \mathrm{CH}=\mathrm{CH}_{2}\right) ; \delta_{\mathrm{C}}\left(125 \mathrm{MHz} ; \mathrm{D}_{2} \mathrm{O}\right) 22.8$ and $23.0\left(2 \times \mathrm{NDCOCH}_{3}\right), 55.1(\mathrm{C}-2), 56.3\left(\mathrm{C}-2^{\prime}\right), 61.4\left(\mathrm{C}-6^{\prime}\right)$, 69.1 (C-4), $69.4(\mathrm{C}-6), 70.5\left(\mathrm{C}-4^{\prime}\right), 71.0\left(\mathrm{OCH}_{2} \mathrm{CH}=\mathrm{CH}_{2}\right)$, $72.4\left(\mathrm{C}-3^{\prime \prime}\right), 73.4\left(\mathrm{C}-2^{\prime \prime}\right), 74.5\left(\mathrm{C}-3^{\prime}\right), 74.8$ (C-5), $75.5\left(\mathrm{C}-4^{\prime \prime}\right)$, $76.3\left(\mathrm{C}-5^{\prime \prime}\right), 76.5$ (C-5'), 83.8 (C-3), 100.5 (C-1), $102.6\left(\mathrm{C}-1^{\prime}\right)$, $103.7\left(\mathrm{C}-1^{\prime \prime}\right), 119.1\left(\mathrm{OCH}_{2} \mathrm{CH}=\mathrm{CH}_{2}\right), 134.0\left(\mathrm{OCH}_{2} \mathrm{CH}=\mathrm{CH}_{2}\right)$; High resolution MALDI-TOF MS, $m / z$ found $\mathrm{M}+\mathrm{H} 641.236$, $\mathrm{C}_{25} \mathrm{H}_{41} \mathrm{~N}_{2} \mathrm{O}_{17}$ requires 641.241.

\section{3-(2-Aminoethylthio)propyl (2-acetamido-2-deoxy- $\beta$-D- glucopyranosyl)-( $(\rightarrow 6)$-I( $\beta$-D-glucopyranosyluronic acid $)-(1 \rightarrow$ 3)|-2-acetamido-2-deoxy- $\beta$-D-glucopyranoside 2}

A solution of $\mathbf{1 3}(3 \mathrm{mg}, 4.7 \mu \mathrm{mol})$ and cysteamine hydrochloride $(3 \mathrm{mg}, 26 \mu \mathrm{mol})$ in water $\left(1 \mathrm{~cm}^{3}\right)$ was irradiated for $2 \mathrm{~h}$ in a quartz vial, using a VL-50C Vilber Lourmat UV Lamp. The mixture was loaded directly on to a size-exclusion column (Bio-Gel P-2, $\left.100 \mathrm{mM} \mathrm{NH}_{4} \mathrm{HCO}_{3}\right)$, yielding $2(1.6 \mathrm{mg}, 48 \%)$, isolated after lyophilization from water as a white, amorphous powder; $[\alpha]_{\mathrm{D}}^{20}-11$ (c 0.1 in water); $\delta_{\mathrm{H}}\left(500 \mathrm{MHz} ; \mathrm{D}_{2} \mathrm{O} ; 2 \mathrm{D}\right.$ TOCSY and HSQC) $1.84\left[2 \mathrm{H}, \mathrm{m}, \mathrm{OCH}_{2} \mathrm{CH}_{2} \mathrm{CH}_{2} \mathrm{~S}\left(\mathrm{CH}_{2}\right)_{2} \mathrm{ND}_{2}\right.$ ], 2.01 and 2.06 (each $3 \mathrm{H}, 2 \times \mathrm{s}, 2 \times \mathrm{NAc}), 2.61\left[2 \mathrm{H}\right.$, br t, $\mathrm{O}\left(\mathrm{CH}_{2}\right)_{2} \mathrm{CH}_{2} \mathrm{~S}\left(\mathrm{CH}_{2}\right)_{2} \mathrm{ND}_{2}$ ], 
2.81 and 3.17 [each $2 \mathrm{H}, 2 \times$ br t, $\mathrm{O}\left(\mathrm{CH}_{2}\right)_{3} \mathrm{~S}\left(\mathrm{CH}_{2}\right)_{2} \mathrm{ND}_{2}$ ], 3.34 (1 H, br t, H-2"), $3.45\left(1 \mathrm{H}, \mathrm{m}, \mathrm{H}-5^{\prime}\right), 3.46(1 \mathrm{H}$, br t, H-4'), $3.50(1 \mathrm{H}$, br t, H-3"), 3.51 (1 H, br t, H-4"), $3.52(1 \mathrm{H}$, br t, $\mathrm{H}-4), 3.54(1 \mathrm{H}$, br t, H-3'), $3.55(1 \mathrm{H}, \mathrm{m}, \mathrm{H}-5), 3.67$ and 3.94 [each $1 \mathrm{H}, 2 \times \mathrm{m}, \mathrm{OCH}_{2}\left(\mathrm{CH}_{2}\right)_{2} \mathrm{~S}\left(\mathrm{CH}_{2}\right)_{2} \mathrm{ND}_{2}$ ], $3.71(1 \mathrm{H}$, br t, $\mathrm{H}-3), 3.73\left(1 \mathrm{H}, \mathrm{d}, \mathrm{H}-5^{\prime \prime}\right), 3.74(1 \mathrm{H}$, br t, $\mathrm{H}-2), 3.75(1 \mathrm{H}, \mathrm{m}$, H-6b'), 3.76 (1 H, br t, H-2'), 3.79 (1 H, m, H-6b), 3.92 (1 $\mathrm{H}$, dd, $\left.J_{\mathrm{H}-5^{\prime}, \mathrm{H}-6 \mathrm{a}^{\prime}} 1.8 \mathrm{~Hz}, J_{\mathrm{H}-6 \mathrm{a}^{\prime}, \mathrm{H}-6 \mathrm{~b}^{\prime}} 12.4 \mathrm{~Hz}, \mathrm{H}-6 \mathrm{a}^{\prime}\right), 4.19(1 \mathrm{H}$, $\left.\mathrm{dd}, J_{\mathrm{H}-5, \mathrm{H}-6 \mathrm{a}} 1.2, J_{\mathrm{H}-6 \mathrm{a}, \mathrm{H}-6 \mathrm{~b}} 11.3, \mathrm{H}-6 \mathrm{a}\right), 4.46\left(1 \mathrm{H}, \mathrm{d}, J_{\mathrm{H}-1^{\prime \prime}, \mathrm{H}-2^{\prime \prime}} 7.8\right.$, $\left.\mathrm{H}-1^{\prime \prime}\right), 4.51\left(1 \mathrm{H}, \mathrm{d}, J_{\mathrm{H} 1, \mathrm{H} 2} 8.3, \mathrm{H}-1\right), 4.52\left(1 \mathrm{H}, \mathrm{d}, J_{\mathrm{H}-1^{\prime}, \mathrm{H}-2^{\prime}} 8.4\right.$, $\left.\mathrm{H}-1^{\prime}\right) ; \delta_{\mathrm{C}}\left(125 \mathrm{MHz} ; \mathrm{D}_{2} \mathrm{O}\right) 23.0$ and $23.1\left(2 \times \mathrm{NDCOCH}_{3}\right), 27.8$ $\left[\mathrm{O}\left(\mathrm{CH}_{2}\right)_{2} \mathrm{CH}_{2} \mathrm{~S}\left(\mathrm{CH}_{2}\right)_{2} \mathrm{ND}_{2}\right], 29.2\left[\mathrm{OCH}_{2} \mathrm{CH}_{2} \mathrm{CH}_{2} \mathrm{~S}\left(\mathrm{CH}_{2}\right)_{2} \mathrm{ND}_{2}\right]$, 29.5 and $39.2\left[\mathrm{O}\left(\mathrm{CH}_{2}\right)_{3} \mathrm{~S}\left(\mathrm{CH}_{2}\right)_{2} \mathrm{ND}_{2}\right], 55.2(\mathrm{C}-2), 56.3\left(\mathrm{C}-2^{\prime}\right)$, $61.4\left(\mathrm{C}-6^{\prime}\right), 69.1(\mathrm{C}-4), 69.2\left[\mathrm{OCH}_{2}\left(\mathrm{CH}_{2}\right)_{2} \mathrm{~S}\left(\mathrm{CH}_{2}\right)_{2} \mathrm{ND}_{2}\right], 69.4$ (C-6), $70.6\left(\mathrm{C}-4^{\prime}\right), 72.4\left(\mathrm{C}-3^{\prime \prime}\right), 73.5\left(\mathrm{C}-2^{\prime \prime}\right), 74.5\left(\mathrm{C}-3^{\prime}\right), 74.8(\mathrm{C}-$ 5), 76.0 (C-4"), 76.5 (C-5"), 76.6 (C-5'), 83.6 (C-3), 101.8 (C-1), $102.8\left(\mathrm{C}-1^{\prime}\right)$, $103.7\left(\mathrm{C}-1^{\prime \prime}\right)$; high resolution MALDI-TOF MS, $m / z$ found $\mathrm{M}+\mathrm{Na} 740.231, \mathrm{C}_{27} \mathrm{H}_{47} \mathrm{~N}_{3} \mathrm{NaO}_{17} \mathrm{~S}$ requires 740.251 .

\section{4-Methoxyphenyl (methyl 2,3,4-tri- $O$-acetyl- $\beta$-D- glucopyranosyluronate)-( $1 \rightarrow 3)$-2-deoxy-2-phthalimido- $\beta$-D- glucopyranoside 15}

A solution of methyl 2,3,4-tri- $O$-acetyl- $\alpha, \beta$-D-glucopyranosyluronate trichloroacetimidate ${ }^{14}(8 ; 0.75 \mathrm{~g}, 1.65 \mathrm{mmol})$ and 4-methoxyphenyl 2-deoxy-4,6- $O$-isopropylidene-2-phthalimido$\beta$-D-glucopyranoside ${ }^{20}(\mathbf{1 4} ; 0.5 \mathrm{~g}, 1.1 \mathrm{mmol})$ in dry $\mathrm{CH}_{2} \mathrm{Cl}_{2}$ $\left(10 \mathrm{~cm}^{3}\right)$, containing activated molecular sieves $(4 \AA, 0.8 \mathrm{~g})$, was stirred for $45 \mathrm{~min}$ at room temperature, then TMSOTf $\left(54 \mathrm{~mm}^{3}\right.$, $0.30 \mathrm{mmol}$ ) was added at $0{ }^{\circ} \mathrm{C}$. The mixture was stirred for $45 \mathrm{~min}$ at $0{ }^{\circ} \mathrm{C}$ and $30 \mathrm{~min}$ at $\mathrm{rt}$, when TLC $\left(95: 5 \mathrm{CH}_{2} \mathrm{Cl}_{2}\right.$-acetone) showed the formation of a new product $\left(R_{\mathrm{f}}=0.33\right)$. After neutralization with dry pyridine and filtration, the solution was washed with $10 \%$ aq. $\mathrm{NaCl}$, dried, filtered, and concentrated. To a solution of the residue in $\mathrm{CH}_{2} \mathrm{Cl}_{2}\left(20 \mathrm{~cm}^{3}\right)$ and water $\left(0.1 \mathrm{~cm}^{3}\right)$ was added TFA $\left(1 \mathrm{~cm}^{3}\right)$, and the mixture was stirred for $1 \mathrm{~h}$, when TLC (9:1 $\mathrm{CH}_{2} \mathrm{Cl}_{2}$-acetone) confirmed the removal of the isopropylidene function $\left(R_{\mathrm{f}}=0.31\right)$. The mixture was washed with saturated aq. $\mathrm{NaHCO}_{3}$, dried, filtered, and concentrated. Column chromatography $\left(9: 1 \mathrm{CH}_{2} \mathrm{Cl}_{2}\right.$-acetone) of the residue gave $15(0.34 \mathrm{~g}, 71 \%)$, isolated as a colorless glass; $[\alpha]_{\mathrm{D}}^{20}+16(c$ 0.7 in $\left.\mathrm{CHCl}_{3}\right) ; \delta_{\mathrm{H}}\left(500 \mathrm{MHz} ; \mathrm{CDCl}_{3} ; 2 \mathrm{D}\right.$ TOCSY and HSQC) $1.60,1.94$, and 2.00 (each $3 \mathrm{H}, 3 \times \mathrm{s}, 3 \times \mathrm{Ac}), 3.64(1 \mathrm{H}, \mathrm{m}, \mathrm{H}-5)$, 3.70 and 3.76 (each $3 \mathrm{H}, 2 \times \mathrm{s}, \mathrm{COOCH}_{3}$ and $\left.\mathrm{C}_{6} \mathrm{H}_{4} \mathrm{OCH} \mathrm{OCH}_{3}\right), 3.73$ $\left(1 \mathrm{H}\right.$, br t, H-4), $3.86\left(1 \mathrm{H}, \mathrm{dd}, J_{\mathrm{H}-5, \mathrm{H}-6 \mathrm{~b}} 5.5 \mathrm{~Hz}, J_{\mathrm{H}-6 \mathrm{a}, \mathrm{H}-6 \mathrm{~b}} 11.9 \mathrm{~Hz}\right.$, $\mathrm{H}-6 \mathrm{~b}), 4.03\left(1 \mathrm{H}, \mathrm{dd}, J_{\mathrm{H}-5, \mathrm{H}-6 \mathrm{a}} 3.4, \mathrm{H}-6 \mathrm{a}\right), 4.07$ (1 H, d, $J_{\mathrm{H}-4^{\prime}, \mathrm{H}-5^{\prime}} 9.9$, $\left.\mathrm{H}-5^{\prime}\right), 4.45\left(1 \mathrm{H}, \mathrm{dd}, J_{\mathrm{H}-1, \mathrm{H}-2} 8.6, J_{\mathrm{H}-2, \mathrm{H}-3} 10.6, \mathrm{H}-2\right), 4.49(1 \mathrm{H}, \mathrm{d}$, $\left.J_{\mathrm{H}-1^{\prime}, \mathrm{H}-2^{\prime}} 7.8, \mathrm{H}^{-1}{ }^{\prime}\right), 4.59\left(1 \mathrm{H}, \mathrm{dd}, J_{\mathrm{H}-3, \mathrm{H}-4} 8.1, \mathrm{H}-3\right), 4.89(1 \mathrm{H}, \mathrm{dd}$, $\left.J_{\mathrm{H}-2^{\prime}, \mathrm{H}-3^{\prime}} 10.7, \mathrm{H}-2^{\prime}\right), 5.07(1 \mathrm{H}$, br t, H-3' $), 5.15\left(1 \mathrm{H}\right.$, br t, H-4' $\left.{ }^{\prime}\right)$, $5.54(1 \mathrm{H}, \mathrm{d}, \mathrm{H}-1), 6.70$ and $6.76\left(\right.$ each $\left.2 \mathrm{H}, 2 \times \mathrm{m}, \mathrm{C}_{6} \mathrm{H}_{4} \mathrm{OCH}_{3}\right)$, 7.78 and 7.87 (each $2 \mathrm{H}, 2 \times \mathrm{m}$, Phth); high resolution MALDITOF MS, $m / z$ found $\mathrm{M}+\mathrm{Na} 754.201, \mathrm{C}_{34} \mathrm{H}_{37} \mathrm{NNaO}_{17}$ requires 754.196.

\section{4-Methoxyphenyl (methyl 2,3,4-tri- $O$-acetyl- $\beta$-D- glucopyranosyluronate)-(1 $\rightarrow 3)-4,6$-di- $O$-acetyl-2-deoxy-2- phthalimido- $\beta$-D-glucopyranoside 16}

To a solution of $\mathbf{1 5}(0.53 \mathrm{~g}, 0.72 \mathrm{mmol})$ in dry $\mathrm{CH}_{2} \mathrm{Cl}_{2}\left(7.5 \mathrm{~cm}^{3}\right)$ and dry pyridine $\left(7.5 \mathrm{~cm}^{3}\right)$ was added acetic anhydride $\left(7.5 \mathrm{~cm}^{3}\right)$. The mixture was stirred overnight, when TLC $\left(9: 1 \mathrm{CH}_{2} \mathrm{Cl}_{2}-\right.$ acetone) showed the reaction to be completed $\left(R_{\mathrm{f}}=0.77\right)$. Then, the mixture was washed with saturated aq. $\mathrm{NaHCO}_{3}$ and $10 \%$ aq. $\mathrm{NaCl}$, dried, filtered, and concentrated. Column chromatography (95:5 $\mathrm{CH}_{2} \mathrm{Cl}_{2}$-acetone) of the residue yielded $16(0.58 \mathrm{~g}, 100 \%)$, isolated as a white foam; $[\alpha]_{\mathrm{D}}^{20}+10(c 1$ in $\left.\mathrm{CHCl}_{3}\right) ; \delta_{\mathrm{H}}\left(500 \mathrm{MHz} ; \mathrm{CDCl}_{3} ; 2 \mathrm{D}\right.$ TOCSY and HSQC) 1.81 , $1.84,1.87,2.02$, and 2.07 (each $3 \mathrm{H}, 5 \times \mathrm{s}, 5 \times \mathrm{Ac}$ ), 3.64 and 3.65 (each $3 \mathrm{H}, 2 \times \mathrm{s}, \mathrm{COOCH}_{3}$ and $\left.\left.\mathrm{C}_{6} \mathrm{H}_{4} \mathrm{OCH}\right)_{3}\right), 3.79(1 \mathrm{H}, \mathrm{d}$, $\left.J_{\mathrm{H}-4^{\prime}, \mathrm{H}-5^{\prime}} 10.1 \mathrm{~Hz}, \mathrm{H}-5^{\prime}\right), 3.80(1 \mathrm{H}, \mathrm{m}, \mathrm{H}-5), 4.11\left(1 \mathrm{H}, \mathrm{dd}, J_{\mathrm{H}-5, \mathrm{H}-6 \mathrm{a}}\right.$ $\left.2.5, J_{\mathrm{H}-6 \mathrm{a}, \mathrm{H}-6 \mathrm{~b}} 12.2, \mathrm{H}-6 \mathrm{a}\right), 4.21\left(1 \mathrm{H}, \mathrm{dd}, J_{\mathrm{H}-5, \mathrm{H}-6 \mathrm{~b}} 5.1, \mathrm{H}-6 \mathrm{~b}\right), 4.24$ $\left(1 \mathrm{H}, \mathrm{d}, J_{\mathrm{H}-1^{\prime}, \mathrm{H}-2^{\prime}} 8.0, \mathrm{H}-1^{\prime}\right), 4.45\left(1 \mathrm{H}, \mathrm{dd}, J_{\mathrm{H}-1, \mathrm{H}-2} 8.4, J_{\mathrm{H}-2, \mathrm{H}-3} 10.7\right.$, $\mathrm{H}-2), 4.69$ (1 H, br t, H-3), $4.70(1 \mathrm{H}$, br t, H-2' $), 4.85(1 \mathrm{H}$, br t, H-3'), 4.98 (1 H, br t, H-4'), 5.09 (1 H, br t, H-4), $5.40(1 \mathrm{H}$, d, H-1), 6.62 and 6.68 (each $\left.2 \mathrm{H}, 2 \times \mathrm{m}, \mathrm{C}_{6} \mathrm{H}_{4} \mathrm{OCH}_{3}\right), 7.74$ and 7.82 (each $2 \mathrm{H}, 2 \times \mathrm{m}$, Phth); $\delta_{\mathrm{C}}\left(125 \mathrm{MHz} ; \mathrm{CDCl}_{3}\right) 20.1,20.3$, 20.4, 20.5, and $20.7\left(5 \times \mathrm{COCH}_{3}\right), 52.6$ and $55.5\left(\mathrm{COOCH}_{3}\right.$ and $\left.\mathrm{C}_{6} \mathrm{H}_{4} \mathrm{OCH}_{3}\right), 55.4$ (C-2), 62.1 (C-6), 68.5 (C-4), 69.5 (C-4'), 71.1 $\left(\mathrm{C}-2^{\prime}\right), 72.0\left(\mathrm{C}-3^{\prime}\right), 72.2$ (2 C) (C-5 and C-5'), 75.5 (C-3), 97.6 (C1), $100.0\left(\mathrm{C}-1^{\prime}\right), 114.4$ and $118.7\left(C_{6} \mathrm{H}_{4} \mathrm{OCH}_{3}\right), 123.7$ and 134.7 $\left[\mathrm{N}(\mathrm{CO})_{2} \mathrm{C}_{6} \mathrm{H}_{4}\right]$; high resolution MALDI-TOF MS, $\mathrm{m} / \mathrm{z}$ found $\mathrm{M}+\mathrm{Na}$ 838.218, $\mathrm{C}_{38} \mathrm{H}_{41} \mathrm{NNaO}_{19}$ requires 838.217.

\section{(Methyl 2,3,4-tri- $O$-acetyl- $\beta$-D-glucopyranosyluronate)-(1 $\rightarrow$ 3)-4,6-di- $O$-acetyl-2-deoxy-2-phthalimido- $\beta$-D-glucopyranosyl trichloroacetimidate 17}

To a solution of $\mathbf{1 6}(0.23 \mathrm{~g}, 0.28 \mathrm{mmol})$ in $1: 1: 1$ tolueneacetonitrile-water $\left(15 \mathrm{~cm}^{3}\right)$ was added ammonium cerium(IV) nitrate $(1.5 \mathrm{~g}, 2.8 \mathrm{mmol})$. The two-phase mixture was vigorously stirred for $45 \mathrm{~min}$, when TLC (95:5 $\mathrm{CH}_{2} \mathrm{Cl}_{2}-$ acetone) showed the disappearance of $\mathbf{1 6}$ and the appearance of a lower moving spot $\left(R_{\mathrm{f}}=0.09\right)$. After dilution with EtOAc, the organic phase was washed with saturated aq. $\mathrm{NaHCO}_{3}$ and $10 \%$ aq. $\mathrm{NaCl}$, dried, filtered, and concentrated. Column chromatography (95:5 $\mathrm{CH}_{2} \mathrm{Cl}_{2}$-acetone) of the residue gave the hemiacetal intermediate isolated as a yellow solid. To a solution of the hemiacetal $(0.15 \mathrm{~g}, 0.20 \mathrm{mmol})$ in dry $\mathrm{CH}_{2} \mathrm{Cl}_{2}$ $\left(4 \mathrm{~cm}^{3}\right)$ and trichloroacetonitrile $\left(0.21 \mathrm{~cm}^{3}, 2.0 \mathrm{mmol}\right)$ was added, at $0{ }^{\circ} \mathrm{C}, 1,8$-diazabicyclo[5.4.0] undec-7-ene $\left(3.4 \mathrm{~mm}^{3}\right.$, $20 \mu \mathrm{mol})$. The mixture was stirred overnight, when TLC (95:5 $\mathrm{CH}_{2} \mathrm{Cl}_{2}$-acetone) showed the formation of a new product $\left(R_{\mathrm{f}}=0.30\right)$. After concentration, column chromatography (95:5 $\mathrm{CH}_{2} \mathrm{Cl}_{2}$-acetone) of the residue yielded $17(0.15 \mathrm{~g}, 63 \%)$, isolated as a yellow foam; $[\alpha]_{\mathrm{D}}^{20}+18\left(c 1\right.$ in $\left.\mathrm{CHCl}_{3}\right) ; \delta_{\mathrm{H}}(500 \mathrm{MHz}$; $\mathrm{CDCl}_{3}$; 2D TOCSY and HSQC) 1.86, 1.91, 1.95, 2.11, and 2.14 (each $3 \mathrm{H}, 5 \times \mathrm{s}, 5 \times \mathrm{Ac}), 3.73\left(3 \mathrm{H}, \mathrm{s}, \mathrm{COOCH}_{3}\right), 3.87(1 \mathrm{H}, \mathrm{d}$, $\left.J_{\mathrm{H}-4^{\prime}, \mathrm{H}-5^{\prime}} 10.0 \mathrm{~Hz}, \mathrm{H}-5^{\prime}\right), 3.97(1 \mathrm{H}, \mathrm{m}, \mathrm{H}-5), 4.18\left(1 \mathrm{H}, \mathrm{dd}, J_{\mathrm{H}-5, \mathrm{H}-6 \mathrm{a}}\right.$ $\left.2.2, J_{\mathrm{H}-6 \mathrm{a}, \mathrm{H}-6 \mathrm{~b}} 12.4, \mathrm{H}-6 \mathrm{a}\right), 4.33\left(1 \mathrm{H}, \mathrm{dd}, J_{\mathrm{H}-5, \mathrm{H}-6 \mathrm{~b}} 4.3, \mathrm{H}-6 \mathrm{~b}\right), 4.34$ $\left(1 \mathrm{H}, \mathrm{d}, J_{\mathrm{H}-1^{\prime}, \mathrm{H}-2^{\prime}} 8.0, \mathrm{H}_{-1}{ }^{\prime}\right), 4.59\left(1 \mathrm{H}, \mathrm{dd}, J_{\mathrm{H}-1, \mathrm{H}-2} 8.9, J_{\mathrm{H}-2, \mathrm{H}-3} 10.7\right.$, $\mathrm{H}-2), 4.76\left(1 \mathrm{H}, \mathrm{dd}, J_{\mathrm{H}-2^{\prime}, \mathrm{H}-3^{\prime}} 9.5, \mathrm{H}-2^{\prime}\right), 4.84\left(1 \mathrm{H}, \mathrm{dd}, J_{\mathrm{H}-3, \mathrm{H}-4} 9.2\right.$, H-3), 4.93 (1 H, br t, H-3'), 5.05 (1 H, br t, H-4'), 5.20 (1 H, br t, H-4), 6.29 (1 H, d, H-1), 7.80 and 7.86 (each $2 \mathrm{H}, 2 \times \mathrm{m}$, Phth), $8.57\left[1 \mathrm{H}, \mathrm{s}, \mathrm{OC}(\mathrm{NH}) \mathrm{CCl}_{3}\right] ; \delta_{\mathrm{C}}\left(125 \mathrm{MHz} ; \mathrm{CDCl}_{3}\right) 20.2,20.3$, 20.6, 20.7, and $20.8\left(5 \times \mathrm{COCH}_{3}\right), 52.7\left(\mathrm{COOCH}_{3}\right), 54.6(\mathrm{C}-2)$, 61.9 (C-6), 68.3 (C-4), $69.6\left(\mathrm{C}-4^{\prime}\right), 71.3\left(\mathrm{C}-2^{\prime}\right), 72.1\left(\mathrm{C}-3^{\prime}\right), 72.5$ $\left(\mathrm{C}-5^{\prime}\right), 73.0(\mathrm{C}-5), 75.4(\mathrm{C}-3), 93.7$ (C-1), $100.2\left(\mathrm{C}-1^{\prime}\right), 123.9$ and $135.0\left[\mathrm{~N}(\mathrm{CO})_{2} C_{6} \mathrm{H}_{4}\right]$.

\section{Allyl (methyl 2,3,4-tri- $O$-acetyl- $\beta$-D- \\ glucopyranosyluronate)-( $\rightarrow 3)-(4,6-d i-O$-acetyl-2-deoxy- \\ 2-phthalimido- $\beta$-D-glucopyranosyl)-( $1 \rightarrow 6)-[($ methyl \\ $2,3,4$-tri- $O$-acetyl- $\beta$-D-glucopyranosyluronate)-( $(1 \rightarrow 3)]$-2-deoxy- 2-phthalimido- $\beta$-D-glucopyranoside 18}

A solution of $9(50 \mathrm{mg}, 75 \mu \mathrm{mol})$ and $17(96 \mathrm{mg}, 112 \mu \mathrm{mol})$ in dry $\mathrm{CH}_{2} \mathrm{Cl}_{2}\left(2 \mathrm{~cm}^{3}\right)$, containing activated molecular sieves ( $4 \AA, 0.1 \mathrm{~g})$, was stirred for $1 \mathrm{~h}$ at $\mathrm{rt}$, then TMSOTf $\left(2.32 \mathrm{~mm}^{3}\right.$, $11.2 \mu \mathrm{mol}$ ) was added at $0{ }^{\circ} \mathrm{C}$. The mixture was stirred for $1 \mathrm{~h}$ at $0{ }^{\circ} \mathrm{C}$, when TLC $\left(9: 1 \mathrm{CH}_{2} \mathrm{Cl}_{2}\right.$-acetone) showed the formation of a new product $\left(R_{\mathrm{f}}=0.42\right)$. After neutralization with dry pyridine and filtration, the solution was washed with $10 \%$ aq. $\mathrm{NaCl}$, dried, filtered, and concentrated. Column chromatography (9:1 $\mathrm{CH}_{2} \mathrm{Cl}_{2}$-acetone) of the residue afforded $\mathbf{1 8}(90 \mathrm{mg}$, $90 \%)$, isolated as a white amorphous powder; $[\alpha]_{\mathrm{D}}^{20}-16(c 1.5 \mathrm{in}$ $\left.\mathrm{CHCl}_{3}\right) ; \delta_{\mathrm{H}}\left(500 \mathrm{MHz} ; \mathrm{CDCl}_{3} ; 2 \mathrm{D}\right.$ TOCSY and HSQC) 1.59 , $1.86,1.90,1.94,1.95,2.00,2.12$, and 2.13 (each $3 \mathrm{H}, 8 \times \mathrm{s}$, $8 \times \mathrm{Ac}), 3.19\left(1 \mathrm{H}, \mathrm{dd}, J_{\mathrm{H}-3, \mathrm{H}-4} 9.6 \mathrm{~Hz}, J_{\mathrm{H}-4, \mathrm{H}-5} 8.4 \mathrm{~Hz}, \mathrm{H}-4\right), 3.40$ $(1 \mathrm{H}, \mathrm{m}, \mathrm{H}-5), 3.65\left(1 \mathrm{H}, \mathrm{dd}, J_{\mathrm{H}-5, \mathrm{H}-6 \mathrm{~b}} 6.0, J_{\mathrm{H}-6 \mathrm{a}, \mathrm{H}-6 \mathrm{~b}} 11.0, \mathrm{H}-6 \mathrm{~b}\right)$, 3.69 and $3.93\left(\right.$ each $\left.1 \mathrm{H}, 2 \times \mathrm{m}, \mathrm{OCH}_{2} \mathrm{CH}=\mathrm{CH}_{2}\right), 3.72$ and 3.76 (each $\left.3 \mathrm{H}, 2 \times \mathrm{s}, 2 \times \mathrm{COOCH}_{3}\right), 3.77\left(1 \mathrm{H}, \mathrm{m}, \mathrm{H}-5^{\prime}\right), 3.86(1 \mathrm{H}$, $\left.\mathrm{d}, J_{\mathrm{H}-4^{\prime \prime}, \mathrm{H}-5^{\prime \prime}} 9.9, \mathrm{H}-5^{\prime \prime}\right), 3.94\left(1 \mathrm{H}, \mathrm{d}, J_{\mathrm{H}-4^{\prime \prime}, \mathrm{H}-5^{\prime \prime \prime}} 10.1, \mathrm{H}-5^{\prime \prime \prime}\right), 3.96$ 
$\left(1 \mathrm{H}, \mathrm{dd}, J_{\mathrm{H}-1, \mathrm{H}-2} 8.6, J_{\mathrm{H}-2, \mathrm{H}-3} 10.7, \mathrm{H}-2\right), 4.18\left(1 \mathrm{H}, \mathrm{dd}, J_{\mathrm{H}-5^{\prime}, \mathrm{H}-6 \mathrm{a}}\right.$ $\left.2.5, J_{\mathrm{H}-6 \mathrm{a}^{\prime}, \mathrm{H}-6 \mathrm{~b}^{\prime}} 12.2, \mathrm{H}-6 \mathrm{a}^{\prime}\right), 4.21\left(1 \mathrm{H}, \mathrm{dd}, J_{\mathrm{H}-5, \mathrm{H}-6 \mathrm{a}} 0.8, \mathrm{H}-6 \mathrm{a}\right), 4.26$ (1 H, dd, H-6b'), 4.27 (1 H, d, $\left.J_{\mathrm{H}-1^{\prime \prime \prime}, \mathrm{H}-2^{\prime \prime \prime}} 8.0, \mathrm{H}-1^{\prime \prime \prime}\right), 4.28$ (1 H, br t, H-3), $4.30\left(1 \mathrm{H}, \mathrm{d}, J_{\mathrm{H}-1^{\prime \prime}, \mathrm{H}-2^{\prime \prime}} 8.1, \mathrm{H}-1^{\prime \prime}\right), 4.32\left(1 \mathrm{H}, \mathrm{dd}, J_{\mathrm{H}-1^{\prime}, \mathrm{H}-2^{\prime}}\right.$ $\left.8.4, J_{\mathrm{H}-2^{\prime}, \mathrm{H}-3^{\prime}} 10.8, \mathrm{H}-2^{\prime}\right), 4.72\left(1 \mathrm{H}, \mathrm{dd}, J_{\mathrm{H}-2^{\prime \prime \prime}, \mathrm{H}-3^{\prime \prime \prime}} 9.7, \mathrm{H}-2^{\prime \prime \prime}\right), 4.73$ $\left(1 \mathrm{H}\right.$, br t, H-3'), $4.76\left(1 \mathrm{H}\right.$, dd, $\left.J_{\mathrm{H}-2^{\prime \prime}, \mathrm{H}-3^{\prime \prime}} 9.2, \mathrm{H}-2^{\prime \prime}\right), 4.80(1 \mathrm{H}$, d, H-1), $4.91(1 \mathrm{H}$, br t, H-3"), 4.94 and 5.00 (each $1 \mathrm{H}, 2 \times \mathrm{m}$, $\left.\mathrm{OCH}_{2} \mathrm{CH}=\mathrm{CH}_{2}\right), 4.99(1 \mathrm{H}$, br t, H-3"'), $5.05(1 \mathrm{H}$, br t, H-4"), $5.08\left(1 \mathrm{H}\right.$, br t, H-4"'), $5.09\left(1 \mathrm{H}, \mathrm{d}, \mathrm{H}-1^{\prime}\right), 5.10\left(1 \mathrm{H}, \mathrm{br} \mathrm{t}, \mathrm{H}-4^{\prime}\right)$, $5.51\left(1 \mathrm{H}, \mathrm{m}, \mathrm{OCH}_{2} \mathrm{CH}=\mathrm{CH}_{2}\right), 7.75,7.82$, and $7.89(2 \mathrm{H}, 4 \mathrm{H}$, and $2 \mathrm{H}, 3 \times \mathrm{m}, 2 \times \mathrm{Phth}) ; \delta_{\mathrm{C}}\left(125 \mathrm{MHz} ; \mathrm{CDCl}_{3}\right) 19.8,20.2$, $20.4(3 \mathrm{C}), 20.5$, and $20.8(2 \mathrm{C})\left(8 \times \mathrm{COCH}_{3}\right), 52.7$ and 53.3 $\left(2 \times \mathrm{COOCH}_{3}\right), 54.7(\mathrm{C}-2), 55.6\left(\mathrm{C}-2^{\prime}\right), 62.3\left(\mathrm{C}-6^{\prime}\right), 68.7(2 \mathrm{C})$ (C-4" and C-4"'), $69.0\left(\mathrm{OCH}_{2} \mathrm{CH}=\mathrm{CH}_{2}\right), 69.2(\mathrm{C}-6), 69.4(\mathrm{C}-4)$, 69.6 (C-4'), 70.7 (C-2"'), 71.2 (C-2"), 71.5 (C-5"'), 71.8 (C-3"'), $72.0\left(\mathrm{C}-5^{\prime}\right), 72.2\left(\mathrm{C}-3^{\prime \prime}\right), 72.4\left(\mathrm{C}-5^{\prime \prime}\right), 74.8(\mathrm{C}-5), 75.7\left(\mathrm{C}-3^{\prime}\right), 81.4$ (C-3), 96.9 (C-1), 99.0 (C-1'), 99.8 (C-1"'), 100.2 (C-1"), 117.6 $\left(\mathrm{OCH}_{2} \mathrm{CH}=\mathrm{CH}_{2}\right), 133.4\left(\mathrm{OCH}_{2} \mathrm{CH}=\mathrm{CH}_{2}\right), 123.8$ and 134.8 $\left[\mathrm{N}(\mathrm{CO})_{2} \mathrm{C}_{6} \mathrm{H}_{4}\right]$; high resolution MALDI-TOF MS, $\mathrm{m} / \mathrm{z}$ found $\mathrm{M}+\mathrm{Na} 1379.358, \mathrm{C}_{61} \mathrm{H}_{68} \mathrm{~N}_{2} \mathrm{NaO}_{33}$ requires 1379.360 .

\section{Allyl ( $\beta$-D-glucopyranosyluronic acid)-( $\rightarrow 3)$-(2-acetamido-2- deoxy- $\beta$-D-glucopyranosyl)-( $\rightarrow 6)-[(\beta$-D-glucopyranosyluronic acid)-( $1 \rightarrow 3)$ |-2-acetamido-2-deoxy- $\beta$-D-glucopyranoside 19}

To a solution of $\mathbf{1 8}(30 \mathrm{mg}, 22 \mu \mathrm{mol})$ in $5: 1 \mathrm{MeOH}$-water $\left(3 \mathrm{~cm}^{3}\right)$ was added, at $0{ }^{\circ} \mathrm{C}, 3 \mathrm{M}$ aq. $\mathrm{NaOH}\left(1 \mathrm{~cm}^{3}\right)$. The mixture was stirred for $3 \mathrm{~h}$ at $\mathrm{rt}$, neutralized with Dowex $50 \mathrm{X} 8 \mathrm{H}^{+}$ resin, filtered, and concentrated. A solution of the residue in 2:1 $n$-butanol-1,2-diaminoethane $\left(6 \mathrm{~cm}^{3}\right)$ was stirred overnight at $90{ }^{\circ} \mathrm{C}$, then co-concentrated with toluene. To a solution of the residue in dry $\mathrm{MeOH}\left(5 \mathrm{~cm}^{3}\right)$ was added, at $0{ }^{\circ} \mathrm{C}$, acetic anhydride $\left(100 \mathrm{~mm}^{3}\right)$. The mixture was stirred for $3 \mathrm{~h}$, then concentrated. Size-exclusion chromatography (Bio-Gel P-2, $\left.100 \mathrm{mM} \mathrm{NH} \mathrm{NCO}_{3}\right)$ gave $19(7.2 \mathrm{mg}, 42 \%)$, isolated after lyophilization from water, as a white amorphous powder; $[\alpha]_{\mathrm{D}}^{20}-33$ ( $c 0.4$ in water); $\delta_{\mathrm{H}}\left(500 \mathrm{MHz} ; \mathrm{D}_{2} \mathrm{O} ; 2 \mathrm{D}\right.$ TOCSY and HSQC) 2.00 and 2.05 (each $3 \mathrm{H}, 2 \times \mathrm{s}, 2 \times \mathrm{NAc}), 3.34(2 \mathrm{H}$, br t, H-2" and H-2"'), 3.73 (2 H, br t, H-3 and H-3'), $3.76(2 \mathrm{H}$, br d, H-5" and $\left.\mathrm{H}-5^{\prime \prime \prime}\right), 3.76$ and 4.23 (each $1 \mathrm{H}, 2 \times \mathrm{m}, 2 \times \mathrm{H}-$ 6), 3.77 and 3.93 (each $\left.1 \mathrm{H}, 2 \times \mathrm{m}, 2 \times \mathrm{H}-6^{\prime}\right), 3.81(1 \mathrm{H}$, br t, $\mathrm{H}-2), 3.89$ (1 H, br t, H-2'), 4.14 and 4.32 (each $1 \mathrm{H}, 2 \times \mathrm{m}$, $\left.\mathrm{OCH}_{2} \mathrm{CH}=\mathrm{CH}_{2}\right), 4.47\left(1 \mathrm{H}, \mathrm{d}, J_{\mathrm{H}-1^{\prime \prime} \mathrm{H}-2^{\prime \prime}} 7.8 \mathrm{~Hz}, \mathrm{H}-1^{\prime \prime}\right), 4.50$ $\left(1 \mathrm{H}, \mathrm{d}, J_{\mathrm{H}-1^{\prime \prime}, \mathrm{H}-2^{\prime \prime \prime}} 7.9, \mathrm{H}-1^{\prime \prime \prime}\right), 4.56\left(1 \mathrm{H}, \mathrm{d}, J_{\mathrm{H}-1^{\prime}, \mathrm{H}-2^{\prime}} 7.8, \mathrm{H}-1^{\prime}\right)$, $4.57\left(1 \mathrm{H}, \mathrm{d}, J_{\mathrm{H}-1, \mathrm{H}-2} 8.5, \mathrm{H}-1\right), 5.28$ and $5.30($ each $1 \mathrm{H}, 2 \times \mathrm{m}$, $\left.\mathrm{OCH}_{2} \mathrm{CH}=\mathrm{CH}_{2}\right), 5.91\left(1 \mathrm{H}, \mathrm{m}, \mathrm{OCH}_{2} \mathrm{CH}=\mathrm{CH}_{2}\right) ; \delta_{\mathrm{C}}(125 \mathrm{MHz}$ $\left.\mathrm{D}_{2} \mathrm{O}\right) 22.7$ and $23.0\left(2 \times \mathrm{NDCOCH}_{3}\right), 55.1(2 \mathrm{C})(\mathrm{C}-2$ and $\mathrm{C}-$ $\left.2^{\prime}\right), 61.4\left(\mathrm{C}-6^{\prime}\right), 69.4(\mathrm{C}-6), 71.1\left(\mathrm{OCH}_{2} \mathrm{CH}=\mathrm{CH}_{2}\right), 73.5(2 \mathrm{C})$ (C-2" and C-2"'), 76.4 (2 C) (C-5" and C-5"'), 83.7 (2 C) (C-3 and C-3'), 100.5 (C-1), 102.4 (C-1'), 103.7 (2 C) (C-1" and C-1"'), $119.1\left(\mathrm{OCH}_{2} \mathrm{CH}=\mathrm{CH}_{2}\right), 134.0\left(\mathrm{OCH}_{2} \mathrm{CH}=\mathrm{CH}_{2}\right)$; high resolution MALDI-TOF MS, $m / z$ found $\mathrm{M}+\mathrm{H}$ 817.280, $\mathrm{C}_{31} \mathrm{H}_{49} \mathrm{~N}_{2} \mathrm{O}_{23}$ requires 817.273.

3-(2-Aminoethylthio)propyl ( $\beta$-D-glucopyranosyluronic acid)$(1 \rightarrow 3)$-(2-acetamido-2-deoxy- $\beta$-D-glucopyranosyl)- $(1 \rightarrow$ 6)-[( $\beta$-D-glucopyranosyluronic acid)-(1 $\rightarrow 3)]$-2-acetamido-2deoxy- $\beta$-D-glucopyranoside 3

A solution of $19 \quad(3 \mathrm{mg}, 3.7 \mu \mathrm{mol})$ and cysteamine hydrochloride ( $3 \mathrm{mg}, 26 \mu \mathrm{mol})$ in water $\left(1 \mathrm{~cm}^{3}\right)$ was irradiated for $2 \mathrm{~h}$ in a quartz vial, using a VL-50C Vilber Lourmat UV Lamp. The mixture was loaded directly on to a size-exclusion column (Bio-Gel P-2, $100 \mathrm{mM} \mathrm{NH}{ }_{4} \mathrm{HCO}_{3}$ ), yielding $3(1.8 \mathrm{mg}, 56 \%$ ), isolated after lyophilization from water as a white, amorphous powder; $[\alpha]_{\mathrm{D}}^{20}-35$ ( $c 0.1$ in water $) ; \delta_{\mathrm{H}}\left(500 \mathrm{MHz} ; \mathrm{D}_{2} \mathrm{O} ; 2 \mathrm{D}\right.$ TOCSY and HSQC) 1.85 [2 $\mathrm{H}$, br t, $\mathrm{OCH}_{2} \mathrm{CH}_{2} \mathrm{CH}_{2} \mathrm{~S}\left(\mathrm{CH}_{2}\right)_{2} \mathrm{ND}_{2}$ ], 2.02 and 2.03 (each $3 \mathrm{H}, 2 \times \mathrm{s}, 2 \times \mathrm{NAc}$ ), $2.61[2 \mathrm{H}$, br t, $\mathrm{O}\left(\mathrm{CH}_{2}\right)_{2} \mathrm{CH}_{2} \mathrm{~S}\left(\mathrm{CH}_{2}\right)_{2} \mathrm{ND}_{2}$ ], 2.82 and 3.18 [each $2 \mathrm{H}, 2 \times$ br t, $\mathrm{O}\left(\mathrm{CH}_{2}\right)_{3} \mathrm{~S}\left(\mathrm{CH}_{2}\right)_{2} \mathrm{ND}_{2}$ ], 3.34 (2 H, br t, H-2" and $\left.\mathrm{H}-2^{\prime \prime \prime}\right), 3.66$ and 3.94 [each $1 \mathrm{H}, 2 \times \mathrm{m}, \mathrm{OCH}_{2}\left(\mathrm{CH}_{2}\right)_{2} \mathrm{~S}\left(\mathrm{CH}_{2}\right)_{2} \mathrm{ND}_{2}$ ], 3.72 (1 H, br t, H-3), 3.77 (1 H, br t, H-3'), 3.73 (2 H, br d, H-5" and $\mathrm{H}-5^{\prime \prime \prime}$ ), 3.76 and 3.93 (each $\left.1 \mathrm{H}, 2 \times \mathrm{m}, 2 \times \mathrm{H}-6^{\prime}\right), 3.77$ ( $1 \mathrm{H}$, br t, H-2), 3.78 and 4.20 (each $1 \mathrm{H}, 2 \times \mathrm{m}, 2 \times \mathrm{H}-6$ ), $4.46\left(1 \mathrm{H}, \mathrm{d}, J_{\mathrm{H}-1^{\prime \prime}, \mathrm{H}-2^{\prime \prime}} 7.0 \mathrm{~Hz}, \mathrm{H}-1^{\prime \prime}\right), 4.48\left(1 \mathrm{H}, \mathrm{d}, J_{\mathrm{H}-1^{\prime \prime}, \mathrm{H}-2^{\prime \prime \prime}}\right.$ 7.5, H-1"') $4.50\left(1 \mathrm{H}, \mathrm{d}, J_{\mathrm{H}-1^{\prime}, \mathrm{H}-2^{\prime}} 8.0, \mathrm{H}-1^{\prime}\right), 4.57(1 \mathrm{H}, \mathrm{d}$, $\left.J_{\mathrm{H}-1, \mathrm{H}-2} 8.5, \mathrm{H}-1\right) ; \delta_{\mathrm{C}}\left(125 \mathrm{MHz} ; \mathrm{D}_{2} \mathrm{O}\right) 22.9\left(\mathrm{NDCOCH}_{3}\right), 27.7$ $\left[\mathrm{O}\left(\mathrm{CH}_{2}\right)_{2} \mathrm{CH}_{2} \mathrm{~S}\left(\mathrm{CH}_{2}\right)_{2} \mathrm{ND}_{2}\right], 29.1\left[\mathrm{OCH}_{2} \mathrm{CH}_{2} \mathrm{CH}_{2} \mathrm{~S}\left(\mathrm{CH}_{2}\right)_{2} \mathrm{ND}_{2}\right]$, 29.2 and $39.1\left[\mathrm{O}\left(\mathrm{CH}_{2}\right)_{3} \mathrm{~S}\left(\mathrm{CH}_{2}\right)_{2} \mathrm{ND}_{2}\right], 55.1\left(\mathrm{C}-2^{\prime}\right), 55.2(\mathrm{C}-2)$, $61.3\left(\mathrm{C}-6^{\prime}\right), 69.1\left[\mathrm{OCH}_{2}\left(\mathrm{CH}_{2}\right)_{2} \mathrm{~S}\left(\mathrm{CH}_{2}\right)_{2} \mathrm{ND}_{2}\right], 69.3(\mathrm{C}-6), 73.5(2$ C) $\left(\mathrm{C}-2^{\prime \prime}\right.$ and $\left.\mathrm{C}-2^{\prime \prime \prime}\right), 76.5(2 \mathrm{C})\left(\mathrm{C}-5^{\prime \prime}\right.$ and $\left.\mathrm{C}-5^{\prime \prime \prime}\right), 83.3\left(\mathrm{C}-3^{\prime}\right), 83.6$ (C-3), 101.9 (C-1), 102.7 (C-1'), 103.6 (2 C) (C-1" and C-1"'); high resolution MALDI-TOF MS, $m / z$ found $\mathrm{M}+\mathrm{Na} 916.252$, $\mathrm{C}_{33} \mathrm{H}_{55} \mathrm{~N}_{3} \mathrm{NaO}_{23} \mathrm{~S}$ requires 916.284.

\section{4-Methoxyphenyl (6-O-levulinoyl-2,3,4-tri- $O$-p-toluoyl- $\beta$-D-glucopyranosyl)-( $\rightarrow 3)$-2-deoxy-2-phthalimido- $\beta$-D- glucopyranoside 22}

A solution of 6-O-levulinoyl-2,3,4-tri- $O-p$-toluoyl- $\alpha$-D-glucopyranosyl trichloroacetimidate ${ }^{15}(\mathbf{2 0} ; 2.33 \mathrm{~g}, 3.0 \mathrm{mmol})$ and 4-methoxyphenyl 4,6- $O$-benzylidene-2-deoxy-2-phthalimido$\beta$-D-glucopyranoside $22(21 ; 1.00 \mathrm{~g}, 2.0 \mathrm{mmol})$ in dry $\mathrm{CH}_{2} \mathrm{Cl}_{2}$ $\left(50 \mathrm{~cm}^{3}\right)$, containing activated molecular sieves $(4 \AA, 1 \mathrm{~g})$, was stirred for $45 \mathrm{~min}$, then TMSOTf $\left(56 \mathrm{~mm}^{3}, 0.3 \mathrm{mmol}\right)$ was added at $0{ }^{\circ} \mathrm{C}$. The mixture was stirred for $30 \mathrm{~min}$, when TLC (95:5 $\mathrm{CH}_{2} \mathrm{Cl}_{2}$-acetone) showed the formation of a new product $\left(R_{\mathrm{f}}=0.42\right)$. After neutralization with dry pyridine and filtration, the solution was washed with $10 \%$ aq. $\mathrm{NaCl}$, dried, filtered, and concentrated. To a solution of the residue in $\mathrm{CH}_{2} \mathrm{Cl}_{2}\left(50 \mathrm{~cm}^{3}\right)$ and water $\left(0.5 \mathrm{~cm}^{3}\right)$ was added TFA $\left(1.5 \mathrm{~cm}^{3}\right)$, then the mixture was stirred for $4 \mathrm{~h}$, when TLC $\left(9: 1 \mathrm{CH}_{2} \mathrm{Cl}_{2}-\mathrm{ac}-\right.$ etone) showed the formation of a new product $\left(R_{\mathrm{f}}=0.38\right)$. The mixture was washed with saturated aq. $\mathrm{NaHCO}_{3}$, dried, filtered, and concentrated. Column chromatography (9:1 $\mathrm{CH}_{2} \mathrm{Cl}_{2}$-acetone) of the residue gave $22(1.57 \mathrm{~g}, 78 \%)$, isolated as a white foam; $[\alpha]_{\mathrm{D}}^{20}+33\left(\right.$ c 0.5 in $\left.\mathrm{CHCl}_{3}\right) ; \delta_{\mathrm{H}}\left(500 \mathrm{MHz} ; \mathrm{CDCl}_{3}\right.$; 2D TOCSY and HSQC) 2.21, 2.23, 2.32, and 2.33 [each $3 \mathrm{H}$, $4 \times \mathrm{s}, 3 \times \mathrm{COC}_{6} \mathrm{H}_{4} \mathrm{CH}_{3}$ and $\left.\mathrm{CO}\left(\mathrm{CH}_{2}\right)_{2} \mathrm{COCH}_{3}\right], 2.65$ and 2.79 [each $2 \mathrm{H}, 2 \times \mathrm{m}, \mathrm{CO}\left(\mathrm{CH}_{2}\right)_{2} \mathrm{COCH}_{3}$ ], $3.64(1 \mathrm{H}, \mathrm{m}, \mathrm{H}-5), 3.68$ $\left(3 \mathrm{H}, \mathrm{s}, \mathrm{C}_{6} \mathrm{H}_{4} \mathrm{OCH} \mathrm{H}_{3}\right), 3.79\left(1 \mathrm{H}, \mathrm{dd}, J_{\mathrm{H}-3, \mathrm{H}-4} 8.1 \mathrm{~Hz}, J_{\mathrm{H}-4, \mathrm{H}-5} 9.6 \mathrm{~Hz}\right.$, $\mathrm{H}-4), 3.87$ and 4.00 (each $1 \mathrm{H}, 2 \times \mathrm{m}, 2 \times \mathrm{H}-6), 4.09(1 \mathrm{H}, \mathrm{m}$, $\left.\mathrm{H}-5^{\prime}\right), 4.21$ (1 H, dd, $\left.J_{\mathrm{H}-5^{\prime}, \mathrm{H}-6 \mathrm{~b}^{\prime}} 7.8, J_{\mathrm{H}-6 \mathrm{a}^{\prime}, \mathrm{H}-6 \mathrm{~b}^{\prime}} 12.2, \mathrm{H}-6 \mathrm{~b}^{\prime}\right), 4.37$ $\left(1 \mathrm{H}, \mathrm{dd}, J_{\mathrm{H}-5^{\prime}, \mathrm{H}-6 \mathrm{a}^{\prime}} 2.3, \mathrm{H}-6 \mathrm{a}^{\prime}\right), 4.44\left(1 \mathrm{H}, \mathrm{dd}, J_{\mathrm{H}-1, \mathrm{H}-2} 8.6, J_{\mathrm{H}-2, \mathrm{H}-3}\right.$ $10.8, \mathrm{H}-2), 4.68\left(1 \mathrm{H}, \mathrm{dd}, J_{\mathrm{H}-3, \mathrm{H}-4} 8.1, \mathrm{H}-3\right), 4.83\left(1 \mathrm{H}, \mathrm{d}, J_{\mathrm{H}-1^{\prime}, \mathrm{H}-2^{\prime}}\right.$ 8.0, H-1'), $5.38\left(1 \mathrm{H}\right.$, br t, H-4'), $5.41\left(1 \mathrm{H}, \mathrm{dd}, J_{\mathrm{H}-2^{\prime}, \mathrm{H}-3^{\prime}} 9.8\right.$, H-2') 5.48 (1 H, d, H-1), $5.72(1 \mathrm{H}$, br t, H-3'), 6.67 (4 H, m, $\mathrm{C}_{6} \mathrm{H}_{4} \mathrm{OCH}_{3}$ ), 6.84, 6.96, 7.13, 7.33, 7.52, and 7.74 (each $2 \mathrm{H}$, $\left.6 \times \mathrm{d}, 3 \times \mathrm{COC}_{6} \mathrm{H}_{4} \mathrm{CH}_{3}\right) ; \delta_{\mathrm{C}}\left(125 \mathrm{MHz} ; \mathrm{CDCl}_{3}\right) 21.6,21.7(2 \mathrm{C})$, and $29.9\left[3 \times \mathrm{COC}_{6} \mathrm{H}_{4} \mathrm{CH}_{3}\right.$ and $\left.\mathrm{CO}\left(\mathrm{CH}_{2}\right)_{2} \mathrm{COCH}_{3}\right], 27.8$ and $37.9\left[\mathrm{CO}\left(\mathrm{CH}_{2}\right)_{2} \mathrm{COCH}_{3}\right], 54.8(\mathrm{C}-2), 55.7\left(\mathrm{C}_{6} \mathrm{H}_{4} \mathrm{OCH}_{3}\right), 62.9$ $\left(\mathrm{C}-6^{\prime}\right), 63.1$ (C-6), $69.1\left(\mathrm{C}-4^{\prime}\right), 70.7$ (C-4), $71.7\left(\mathrm{C}-2^{\prime}\right), 72.3$ (C$\left.3^{\prime}\right), 72.6$ (C-5'), 75.9 (C-5), 82.8 (C-3), 97.6 (C-1), 101.6 (C-1'), 114.6 and $118.2\left(C_{6} \mathrm{H}_{4} \mathrm{OCH}_{3}\right), 123.8$ and $133.7\left[\mathrm{~N}(\mathrm{CO})_{2} C_{6} \mathrm{H}_{4}\right]$, 129.0, 129.1, 129.4, 129.8 (2 C), and $130.0\left(\mathrm{COC}_{6} \mathrm{H}_{4} \mathrm{CH}_{3}\right)$; high resolution MALDI-TOF MS, $\mathrm{m} / \mathrm{z}$ found $\mathrm{M}+\mathrm{Na} 1052.332$, $\mathrm{C}_{56} \mathrm{H}_{55} \mathrm{NNaO}_{18}$ requires 1052.332.

\section{4-Methoxyphenyl (6- $O$-levulinoyl-2,3,4-tri- $O$-p-toluoyl- $\beta$-D- glucopyranosyl)-(1 $\rightarrow 3)-6$-O-tert-butyldiphenylsilyl-2-deoxy-2- phthalimido- $\beta$-D-glucopyranoside 23}

To a solution of $22(1.3 \mathrm{~g}, 1.26 \mathrm{mmol})$ in dry $\mathrm{CH}_{2} \mathrm{Cl}_{2}\left(50 \mathrm{~cm}^{3}\right)$, containing pyridine $\left(3.6 \mathrm{~cm}^{3}\right)$, triethylamine $\left(1.8 \mathrm{~cm}^{3}\right)$, and a catalytic amount of DMAP, was added tert-butyldiphenylsilyl chloride $\left(0.96 \mathrm{~cm}^{3}, 3.79 \mathrm{mmol}\right)$. The mixture was stirred overnight, when TLC $\left(95: 5 \mathrm{CH}_{2} \mathrm{Cl}_{2}\right.$-acetone) showed the reaction to be completed $\left(R_{\mathrm{f}}=0.82\right)$. After dilution with EtOAc, the solution was washed with saturated aq. $\mathrm{NaHCO}_{3}$ and water, dried, filtered, and concentrated. Column chromatography (toluene $\rightarrow$ 9: $1 \mathrm{CH}_{2} \mathrm{Cl}_{2}$-acetone) of the residue yielded 23 (1.46 g, 91\%), isolated as a white foam; $[\alpha]_{\mathrm{D}}^{20}+19\left(c 1.5\right.$ in $\left.\mathrm{CHCl}_{3}\right) ; \delta_{\mathrm{H}}(500 \mathrm{MHz}$; $\mathrm{CDCl}_{3} ; 2 \mathrm{D}$ TOCSY and HSQC) $1.06\left[9 \mathrm{H}, \mathrm{s}, \mathrm{SiC}\left(\mathrm{CH}_{3}\right)_{3}\left(\mathrm{C}_{6} \mathrm{H}_{5}\right)_{2}\right]$, $2.10,2.22,2.32$, and 2.33 [each $3 \mathrm{H}, 4 \times \mathrm{s}, 3 \times \mathrm{COC}_{6} \mathrm{H}_{4} \mathrm{CH}_{3}$ and 
$\left.\mathrm{CO}\left(\mathrm{CH}_{2}\right)_{2} \mathrm{COCH}_{3}\right], 2.57$ [4 H, m, $\left.\mathrm{CO}\left(\mathrm{CH}_{2}\right)_{2} \mathrm{COCH}_{3}\right], 3.65(3 \mathrm{H}$, $\left.\mathrm{s}, \mathrm{C}_{6} \mathrm{H}_{4} \mathrm{OC} H_{3}\right), 3.71(2 \mathrm{H}, \mathrm{m}, \mathrm{H}-5$ and $\mathrm{H}-4), 3.93\left(1 \mathrm{H}, \mathrm{dd}, J_{\mathrm{H}-5, \mathrm{H}-6 \mathrm{~b}}\right.$ $\left.6.1 \mathrm{~Hz}, J_{\mathrm{H}-6 \mathrm{a}, \mathrm{H}-6 \mathrm{~b}} 11.0 \mathrm{~Hz}, \mathrm{H}-6 \mathrm{~b}\right), 4.06\left(1 \mathrm{H}, \mathrm{m}, \mathrm{H}-5^{\prime}\right), 4.10(1 \mathrm{H}$, $\left.\mathrm{dd}, J_{\mathrm{H}-5, \mathrm{H}-6 \mathrm{a}} 1.7, \mathrm{H}-6 \mathrm{a}\right), 4.19\left(1 \mathrm{H}, \mathrm{dd}, J_{\mathrm{H}-5^{\prime}, \mathrm{H}-6 \mathrm{~b}^{\prime}} 7.7, J_{\mathrm{H}-6 \mathrm{a}^{\prime}, \mathrm{H}-6 \mathrm{~b}^{\prime}} 12.0\right.$, $\left.\mathrm{H}-6 \mathrm{~b}^{\prime}\right), 4.34\left(1 \mathrm{H}, \mathrm{dd}, J_{\mathrm{H}-5^{\prime}, \mathrm{H}-6 \mathrm{a}^{\prime}} 2.3, \mathrm{H}-6 \mathrm{a}^{\prime}\right), 4.46\left(1 \mathrm{H}, \mathrm{dd}, J_{\mathrm{H} 1, \mathrm{H} 2}\right.$ 8.4, $\left.J_{\mathrm{H}-2, \mathrm{H}-3} 10.8, \mathrm{H}-2\right), 4.67\left(1 \mathrm{H}, \mathrm{dd}, J_{\mathrm{H}-3, \mathrm{H}-4} 7.3, \mathrm{H}-3\right), 4.83(1 \mathrm{H}$, d, $\left.J_{\mathrm{H}-1^{\prime}, \mathrm{H}-2^{\prime}} 7.8, \mathrm{H}-1^{\prime}\right), 5.36\left(1 \mathrm{H}\right.$, br t, $\left.\mathrm{H}-4^{\prime}\right), 5.40\left(1 \mathrm{H}, \mathrm{dd}, J_{\mathrm{H}-2^{\prime}, \mathrm{H}-3^{\prime}}\right.$ 9.8, $\left.\mathrm{H}-2^{\prime}\right), 5.43(1 \mathrm{H}, \mathrm{d}, \mathrm{H}-1), 5.71\left(1 \mathrm{H}, \mathrm{br} \mathrm{t}, \mathrm{H}-3^{\prime}\right), 6.59$ and 6.78 (each $2 \mathrm{H}, 2 \times \mathrm{m}, \mathrm{C}_{6} \mathrm{H}_{4} \mathrm{OCH}_{3}$ ), 6.85, 6.96, 7.12, 7.27, 7.52, and $7.73\left(\right.$ each $\left.2 \mathrm{H}, 6 \times \mathrm{d}, 3 \times \mathrm{COC}_{6} \mathrm{H}_{4} \mathrm{CH}_{3}\right), 7.38$ and $7.71[6 \mathrm{H}$ and $\left.4 \mathrm{H}, 2 \times \mathrm{m}, \mathrm{SiC}\left(\mathrm{CH}_{3}\right)_{3}\left(\mathrm{C}_{6} \mathrm{H}_{5}\right)_{2}\right] ; \delta_{\mathrm{C}}\left(125 \mathrm{MHz} ; \mathrm{CDCl}_{3}\right) 21.5,21.6$ (2 C), and $29.7\left[3 \times \mathrm{COC}_{6} \mathrm{H}_{4} \mathrm{CH}_{3}\right.$ and $\left.\mathrm{CO}\left(\mathrm{CH}_{2}\right)_{2} \mathrm{COCH}_{3}\right], 26.8$ $\left[\mathrm{SiC}\left(\mathrm{CH}_{3}\right)_{3}\left(\mathrm{C}_{6} \mathrm{H}_{5}\right)_{2}\right], 27.8$ and $37.8\left[\mathrm{CO}\left(\mathrm{CH}_{2}\right)_{2} \mathrm{COCH}_{3}\right], 54.9(\mathrm{C}-$ 2), $55.6\left(\mathrm{C}_{6} \mathrm{H}_{4} \mathrm{OCH} \mathrm{H}_{3}\right), 62.8\left(\mathrm{C}-6^{\prime}\right), 63.6(\mathrm{C}-6), 69.1\left(\mathrm{C}-4^{\prime}\right), 70.0$ (C-4), $71.6\left(\mathrm{C}-2^{\prime}\right), 72.4\left(\mathrm{C}-3^{\prime}\right), 72.5\left(\mathrm{C}-5^{\prime}\right), 77.0(\mathrm{C}-5), 83.2(\mathrm{C}-3)$, $97.7(\mathrm{C}-1), 101.6\left(\mathrm{C}-1^{\prime}\right), 114.5$ and $118.4\left(C_{6} \mathrm{H}_{4} \mathrm{OCH}_{3}\right), 123.8$ and $133.6\left[\mathrm{~N}(\mathrm{CO})_{2} C_{6} \mathrm{H}_{4}\right], 127.8,129.6$, and $135.8\left[\mathrm{SiC}\left(\mathrm{CH}_{3}\right)_{3}\left(C_{6} \mathrm{H}_{5}\right)_{2}\right]$, 128.9, 129.0, 129.3, $129.7(2 \mathrm{C})$, and $129.9\left(\mathrm{COC}_{6} \mathrm{H}_{4} \mathrm{CH}_{3}\right)$; high resolution MALDI-TOF MS, $\mathrm{m} / \mathrm{z}$ found $\mathrm{M}+\mathrm{Na}$ 1290.456, $\mathrm{C}_{72} \mathrm{H}_{73} \mathrm{NNaO}_{18} \mathrm{Si}$ requires 1290.450 .

\section{4-Methoxyphenyl (6-O-levulinoyl-2,3,4-tri- $O$-p-toluoyl- $\beta$-D- glucopyranosyl)-(1 $\rightarrow 3)-4-O$-acetyl-6-O-tert-butyldiphenylsilyl- 2-deoxy-2-phthalimido- $\beta$-D-galactopyranoside 24}

To a solution of $23(1.4 \mathrm{~g}, 1.1 \mathrm{mmol})$ in dry $\mathrm{CH}_{2} \mathrm{Cl}_{2}\left(50 \mathrm{~cm}^{3}\right)$, containing pyridine $\left(1.18 \mathrm{~cm}^{3}\right)$ and a catalytic amount of DMAP, was added slowly at $0{ }^{\circ} \mathrm{C}$ a solution of trifluoromethanesulfonic anhydride $\left(1.1 \mathrm{~cm}^{3}, 6.6 \mathrm{mmol}\right)$ in dry $\mathrm{CH}_{2} \mathrm{Cl}_{2}$ $\left(6 \mathrm{~cm}^{3}\right)$. The mixture was stirred for $30 \mathrm{~min}$ at $0{ }^{\circ} \mathrm{C}$ and for $5 \mathrm{~h}$ at $\mathrm{rt}$, when TLC $\left(95: 5 \mathrm{CH}_{2} \mathrm{Cl}_{2}\right.$-acetone) showed the triflation to be complete $\left(R_{\mathrm{f}}=0.55\right)$. The mixture was washed with saturated aq. $\mathrm{NaHCO}_{3}$ and $10 \%$ aq. $\mathrm{NaCl}$, dried, filtered, and concentrated. To a solution of the residue in dry DMF $\left(50 \mathrm{~cm}^{3}\right)$ was added tetrabutylammonium acetate $(1.32 \mathrm{~g}, 4.35 \mathrm{mmol})$. After $2 \mathrm{~h}$, when TLC $\left(95: 5 \mathrm{CH}_{2} \mathrm{Cl}_{2}\right.$-acetone) showed the formation of a new product $\left(R_{\mathrm{f}}=0.51\right)$, the mixture was co-concentrated with toluene. A solution of the residue in $\mathrm{CH}_{2} \mathrm{Cl}_{2}\left(50 \mathrm{~cm}^{3}\right)$ was washed with $10 \%$ aq. $\mathrm{NaCl}$, dried, filtered, and concentrated. Column chromatography $\left(95: 5 \mathrm{CH}_{2} \mathrm{Cl}_{2}\right.$-acetone $)$ of the residue afforded $24(1.0 \mathrm{~g}, 70 \%)$, isolated as a yellow foam; $[\alpha]_{\mathrm{D}}^{20}+18(c 0.6$ in $\left.\mathrm{CHCl}_{3}\right) ; \delta_{\mathrm{H}}\left(500 \mathrm{MHz} ; \mathrm{CDCl}_{3} ; 2 \mathrm{D}\right.$ TOCSY and HSQC) 1.05 [9 H, s, $\left.\mathrm{SiC}\left(\mathrm{CH}_{3}\right)_{3}\left(\mathrm{C}_{6} \mathrm{H}_{5}\right)_{2}\right], 2.12,2.13,2.24,2.32$, and 2.33 [each $3 \mathrm{H}, 5 \times \mathrm{s}, 3 \times \mathrm{COC}_{6} \mathrm{H}_{4} \mathrm{CH}_{3}, \mathrm{COCH}_{3}$, and $\left.\mathrm{CO}\left(\mathrm{CH}_{2}\right)_{2} \mathrm{COCH}_{3}\right]$, $2.58\left[4 \mathrm{H}, \mathrm{m}, \mathrm{CO}\left(\mathrm{CH}_{2}\right)_{2} \mathrm{COCH}_{3}\right], 3.64\left(3 \mathrm{H}, \mathrm{s}, \mathrm{C}_{6} \mathrm{H}_{4} \mathrm{OCH} \mathrm{H}_{3}\right), 3.76$ $\left(1 \mathrm{H}, \mathrm{dd}, J_{\mathrm{H}-5, \mathrm{H}-6 \mathrm{~b}} 7.5 \mathrm{~Hz}, J_{\mathrm{H}-6 \mathrm{a}, \mathrm{H}-6 \mathrm{~b}} 10.9 \mathrm{~Hz}, \mathrm{H}-6 \mathrm{~b}\right), 3.81(1 \mathrm{H}$, dd, $\left.J_{\mathrm{H}-5, \mathrm{H}-6 \mathrm{a}} 4.3, \mathrm{H}-6 \mathrm{a}\right), 3.89$ (1 H, m, H-5' $), 4.02$ (1 H, m, H-5), $4.17\left(1 \mathrm{H}, \mathrm{dd}, J_{\mathrm{H}-5^{\prime}, \mathrm{H}-6 \mathrm{~b}^{\prime}} 5.0, J_{\mathrm{H}-6 \mathrm{a}^{\prime}, \mathrm{H}-6 \mathrm{~b}^{\prime}} 12.1, \mathrm{H}-6 \mathrm{~b}^{\prime}\right), 4.31(1 \mathrm{H}, \mathrm{dd}$, $\left.J_{\mathrm{H}-5^{\prime}, \mathrm{H}-6 \mathrm{a}^{\prime}} 2.8, \mathrm{H}-6 \mathrm{a}^{\prime}\right), 4.73\left(1 \mathrm{H}, \mathrm{dd}, J_{\mathrm{H}-1, \mathrm{H}-2} 8.4, J_{\mathrm{H}-2, \mathrm{H}-3} 11.2, \mathrm{H}-2\right)$, $4.78\left(1 \mathrm{H}, \mathrm{d}, J_{\mathrm{H}-1^{\prime}, \mathrm{H}-2^{\prime}} 8.0, \mathrm{H}-1^{\prime}\right), 4.92\left(1 \mathrm{H}, \mathrm{dd}, J_{\mathrm{H}-3, \mathrm{H}-4} 3.3, \mathrm{H}-3\right)$, $5.25\left(1 \mathrm{H}, \mathrm{dd}, J_{\mathrm{H}-2^{\prime}, \mathrm{H}-3^{\prime}} 9.8, \mathrm{H}-2^{\prime}\right), 5.42(1 \mathrm{H}$, br t, H-4' $), 5.52$ (1 H, d, H-1), 5.61 (1 H, br t, H-3'), $5.62(1 \mathrm{H}$, br d, H-4), 6.58 and 6.79 (each $\left.2 \mathrm{H}, 2 \times \mathrm{m}, \mathrm{C}_{6} \mathrm{H}_{4} \mathrm{OCH}_{3}\right), 6.88,6.98,7.11,7.35$, 7.55 , and 7.74 (each $\left.2 \mathrm{H}, 6 \times \mathrm{d}, 3 \times \mathrm{COC}_{6} \mathrm{H}_{4} \mathrm{CH}_{3}\right), 7.40$ and 7.52 [ $6 \mathrm{H}$ and $\left.4 \mathrm{H}, 2 \times \mathrm{m}, \mathrm{SiC}\left(\mathrm{CH}_{3}\right)_{3}\left(\mathrm{C}_{6} \mathrm{H}_{5}\right)_{2}\right] ; \delta_{\mathrm{C}}\left(125 \mathrm{MHz} ; \mathrm{CDCl}_{3}\right)$ 20.9, 21.5, $21.6\left(2 \mathrm{C}\right.$ ), and $29.8\left[3 \times \mathrm{COC}_{6} \mathrm{H}_{4} \mathrm{CH}_{3}, \mathrm{COCH}_{3}\right.$, and $\left.\mathrm{CO}\left(\mathrm{CH}_{2}\right)_{2} \mathrm{COCH}_{3}\right], 26.8\left[\mathrm{SiC}\left(\mathrm{CH}_{3}\right)_{3}\left(\mathrm{C}_{6} \mathrm{H}_{5}\right)_{2}\right], 27.8$ and 37.8 $\left[\mathrm{CO}\left(\mathrm{CH}_{2}\right)_{2} \mathrm{COCH}_{3}\right], 52.6(\mathrm{C}-2), 55.6\left(\mathrm{C}_{6} \mathrm{H}_{4} \mathrm{OCH}_{3}\right), 62.4\left(\mathrm{C}-6^{\prime}\right)$, $63.1(\mathrm{C}-6), 69.0\left(\mathrm{C}-4^{\prime}\right), 69.3(\mathrm{C}-4), 71.8\left(\mathrm{C}-2^{\prime}\right), 72.2\left(\mathrm{C}-5^{\prime}\right), 72.5$ (C-3'), 74.8 (C-3), 75.4 (C-5), 97.9 (C-1), $101.4\left(\mathrm{C}-1 '^{\prime}\right), 114.5$ and $118.3\left(C_{6} \mathrm{H}_{4} \mathrm{OCH}_{3}\right), 123.5$ and $134.0\left[\mathrm{~N}(\mathrm{CO})_{2} C_{6} \mathrm{H}_{4}\right], 127.8$, 129.7, and $135.7\left[\mathrm{SiC}\left(\mathrm{CH}_{3}\right)_{3}\left(C_{6} \mathrm{H}_{5}\right)_{2}\right], 128.9,129.0,129.2,129.8$, 129.9, and $130.0\left(\mathrm{COC}_{6} \mathrm{H}_{4} \mathrm{CH}_{3}\right)$; high resolution MALDI-TOF $\mathrm{MS}, \mathrm{m} / \mathrm{z}$ found $\mathrm{M}+\mathrm{Na} 1332.445, \mathrm{C}_{74} \mathrm{H}_{75} \mathrm{NNaO}_{19} \mathrm{Si}$ requires 1332.460 .

\section{5-Azidopentyl (6-O-levulinoyl-2,3,4-tri- $O$-p-toluoyl- $\beta$-D- glucopyranosyl)-(1 $\rightarrow 3)-4-O$-acetyl-6-O-tert-butyldiphenylsilyl- 2-deoxy-2-phthalimido- $\beta$-D-galactopyranoside 26}

To a solution of $\mathbf{2 4}(0.90 \mathrm{~g}, 0.68 \mathrm{mmol})$ in $1: 1: 1$ toluene-acetonitrile-water $\left(60 \mathrm{~cm}^{3}\right)$ was added ammonium cerium(IV) nitrate
(3.73 g, $6.8 \mathrm{mmol})$. The two-phase mixture was stirred for $2 \mathrm{~h}$, when TLC (95:5 $\mathrm{CH}_{2} \mathrm{Cl}_{2}$-acetone) showed the disappearance of 24. The mixture was diluted with EtOAc, and the organic phase was washed with saturated aq. $\mathrm{NaHCO}_{3}$ and $10 \%$ aq. $\mathrm{NaCl}$, dried, filtered, and concentrated. Column chromatography (95:5 $\mathrm{CH}_{2} \mathrm{Cl}_{2}$-acetone) of the residue gave the hemiacetal intermediate, isolated as a yellow, amorphous solid. To a solution of the hemiacetal $(0.60 \mathrm{~g}, 0.5 \mathrm{mmol})$ in dry $\mathrm{CH}_{2} \mathrm{Cl}_{2}\left(5 \mathrm{~cm}^{3}\right)$ and trichloroacetonitrile $\left(0.55 \mathrm{~cm}^{3}, 5 \mathrm{mmol}\right)$ was added, at $0{ }^{\circ} \mathrm{C}, 1,8$ diazabicyclo[5.4.0]undec-7-ene $\left(8.4 \mathrm{~mm}^{3}, 0.05 \mathrm{mmol}\right)$. After $3 \mathrm{~h}$, the mixture was concentrated and the residue was subjected to column chromatography (95:5 $\mathrm{CH}_{2} \mathrm{Cl}_{2}$-acetone), yielding 25 $(0.57 \mathrm{~g}, 61 \%)$, isolated as a yellow foam; $\delta_{\mathrm{H}}\left(500 \mathrm{MHz} ; \mathrm{CDCl}_{3}\right.$; 2D TOCSY and HSQC) $1.04\left[9 \mathrm{H}, \mathrm{s}, \mathrm{SiC}\left(\mathrm{CH}_{3}\right)_{3}\left(\mathrm{C}_{6} \mathrm{H}_{5}\right)_{2}\right]$, $2.13,2.16,2.24$, and $2.34[3 \mathrm{H}, 3 \mathrm{H}, 3 \mathrm{H}$, and $6 \mathrm{H}, 4 \times \mathrm{s}$, $3 \times \mathrm{COC}_{6} \mathrm{H}_{4} \mathrm{CH}_{3}, \mathrm{COCH}_{3}$, and $\left.\mathrm{CO}\left(\mathrm{CH}_{2}\right)_{2} \mathrm{COCH}_{3}\right], 2.64[4 \mathrm{H}$, $\left.\mathrm{m}, \mathrm{CO}\left(\mathrm{CH}_{2}\right)_{2} \mathrm{COCH}_{3}\right], 3.77(2 \mathrm{H}, \mathrm{m}, 2 \times \mathrm{H}-6), 3.91(1 \mathrm{H}, \mathrm{m}, \mathrm{H}-$ $\left.5^{\prime}\right), 4.11(1 \mathrm{H}, \mathrm{m}, \mathrm{H}-5), 4.20\left(1 \mathrm{H}, \mathrm{dd}, J_{\mathrm{H}-5^{\prime}, \mathrm{H}-6 \mathrm{~b}^{\prime}} 5.1 \mathrm{~Hz}, J_{\mathrm{H}-6 \mathrm{a}^{\prime}, \mathrm{H}-6 \mathrm{~b}^{\prime}}\right.$ $\left.12.3 \mathrm{~Hz}, \mathrm{H}-6 \mathrm{~b}^{\prime}\right), 4.32$ (1 H, dd, $\left.J_{\mathrm{H}-5^{\prime}, \mathrm{H}-6 \mathrm{a}^{\prime}} 2.8, \mathrm{H}-6 \mathrm{a}^{\prime}\right), 4.74(1 \mathrm{H}$, $\left.\mathrm{dd}, J_{\mathrm{H}-1, \mathrm{H}-2} 9.0, J_{\mathrm{H}-2, \mathrm{H}-3} 10.8, \mathrm{H}-2\right), 4.78\left(1 \mathrm{H}, \mathrm{d}, J_{\mathrm{H}-1^{\prime}, \mathrm{H}-2^{\prime}} 7.5, \mathrm{H}-1^{\prime}\right)$, $5.00\left(1 \mathrm{H}, \mathrm{dd}, J_{\mathrm{H}-3, \mathrm{H}-4} 3.3, \mathrm{H}-3\right), 5.27\left(1 \mathrm{H}, \mathrm{dd}, J_{\mathrm{H}-2^{\prime}, \mathrm{H}-3^{\prime}} 9.9, \mathrm{H}-2^{\prime}\right)$, 5.44 (1 H, br t, H-4'), 5.65 (1 H, br t, H-3'), 5.74 (1 H, d, H-4), 6.07 (1 H, d, H-1), 6.88, 6.99, 7.13, 7.33, 7.56, and 7.74 (each $\left.2 \mathrm{H}, 6 \times \mathrm{d}, 3 \times \mathrm{COC}_{6} \mathrm{H}_{4} \mathrm{CH}_{3}\right), 7.40$ and $7.65[6 \mathrm{H}$ and $4 \mathrm{H}, 2 \times \mathrm{m}$, $\left.\mathrm{SiC}\left(\mathrm{CH}_{3}\right)_{3}\left(\mathrm{C}_{6} H_{5}\right)_{2}\right], 8.48\left[1 \mathrm{H}, \mathrm{s}, \mathrm{OC}(\mathrm{NH}) \mathrm{CCl}_{3}\right]$.

A solution of $25(256 \mathrm{mg}, 0.19 \mathrm{mmol})$ and 5-azidopentanol (49 $\mathrm{mg}, 0.38 \mathrm{mmol})$ in dry $\mathrm{CH}_{2} \mathrm{Cl}_{2}\left(2 \mathrm{~cm}^{3}\right)$, containing activated molecular sieves ( $4 \AA, 0.3 \mathrm{~g}$ ), was stirred for $30 \mathrm{~min}$, then TMSOTf $\left(5.1 \mathrm{~mm}^{3}, 0.028 \mathrm{mmol}\right)$ was added at $0{ }^{\circ} \mathrm{C}$, and the mixture was stirred for $30 \mathrm{~min}$. After neutralization with pyridine and filtration, the solution was washed with $10 \%$ aq. $\mathrm{NaCl}$, dried, filtered, and concentrated. Column chromatography ( $95: 5 \mathrm{CH}_{2} \mathrm{Cl}_{2}$-acetone) of the residue gave $\mathbf{2 6}(213 \mathrm{mg}$, $85 \%)$, isolated as a glass; $[a]_{\mathrm{D}}^{20}-5\left(c 2\right.$ in $\left.\mathrm{CHCl}_{3}\right) ; \delta_{\mathrm{H}}(500 \mathrm{MHz}$; $\mathrm{CDCl}_{3} ; 2 \mathrm{D}$ TOCSY and HSQC) $0.99,1.25,1.37$, and 2.83 [each $\left.2 \mathrm{H}, 4 \times \mathrm{m}, \mathrm{OCH}_{2}\left(\mathrm{CH}_{2}\right)_{4} \mathrm{~N}_{3}\right], 1.03\left[9 \mathrm{H}, \mathrm{s}, \mathrm{SiC}\left(\mathrm{CH}_{3}\right)_{3}\left(\mathrm{C}_{6} \mathrm{H}_{5}\right)_{2}\right]$, 2.11, 2.14, 2.17, and $2.32[3 \mathrm{H}, 3 \mathrm{H}, 3 \mathrm{H}$, and $6 \mathrm{H}, 4 \times \mathrm{s}$, $3 \times \mathrm{COC}_{6} \mathrm{H}_{4} \mathrm{CH}_{3}, \mathrm{COCH}_{3}$, and $\left.\mathrm{CO}\left(\mathrm{CH}_{2}\right)_{2} \mathrm{COCH}_{3}\right], 2.54$ and 2.60 [each $2 \mathrm{H}, 2 \times \mathrm{m}, \mathrm{CO}\left(\mathrm{CH}_{2}\right)_{2} \mathrm{COCH}_{3}$ ], 3.29 and 3.78 [each $\left.1 \mathrm{H}, 2 \times \mathrm{m}, \mathrm{OCH}_{2}\left(\mathrm{CH}_{2}\right)_{4} \mathrm{~N}_{3}\right], 3.79(2 \mathrm{H}, \mathrm{m}, 2 \times \mathrm{H}-6), 3.90$ $\left(2 \mathrm{H}, \mathrm{m}, \mathrm{H}-5\right.$ and $\left.\mathrm{H}-5^{\prime}\right), 4.19\left(1 \mathrm{H}, \mathrm{dd}, J_{\mathrm{H}-5^{\prime}, \mathrm{H}-6 \mathrm{~b}^{\prime}} 4.8 \mathrm{~Hz}, J_{\mathrm{H}-6 \mathrm{a}^{\prime}, \mathrm{H}-6 \mathrm{~b}^{\prime}}\right.$ $\left.12.0 \mathrm{~Hz}, \mathrm{H}-6 \mathrm{~b}^{\prime}\right), 4.30$ (1 H, dd, $\left.J_{\mathrm{H}-5^{\prime}, \mathrm{H}-6 \mathrm{a}^{\prime}} 2.7, \mathrm{H}-6 \mathrm{a}^{\prime}\right), 4.46(1 \mathrm{H}$, $\left.\mathrm{dd}, J_{\mathrm{H}-1, \mathrm{H}-2} 8.4, J_{\mathrm{H}-2, \mathrm{H}-3} 11.1, \mathrm{H}-2\right), 4.77\left(1 \mathrm{H}, \mathrm{dd}, J_{\mathrm{H}-1^{\prime}, \mathrm{H}-2^{\prime}} 7.5\right.$, $\left.\mathrm{H}-1^{\prime}\right), 4.82\left(1 \mathrm{H}, \mathrm{dd}, J_{\mathrm{H}-3, \mathrm{H}-4} 3.0, \mathrm{H}-3\right), 5.00(1 \mathrm{H}, \mathrm{d}, \mathrm{H}-1), 5.25$ $\left(1 \mathrm{H}, \mathrm{dd}, J_{\mathrm{H}-2^{\prime}, \mathrm{H}-3^{\prime}} 9.6, \mathrm{H}-2^{\prime}\right), 5.43(1 \mathrm{H}$, br t, H-4' $), 5.64(1 \mathrm{H}$, br t, H-3'), 5.65 (1 H, br d, H-4), 6.88, 6.99, 7.13, 7.38, 7.55, and 7.73 (each $\left.2 \mathrm{H}, 6 \times \mathrm{d}, 3 \times \mathrm{COC}_{6} \mathrm{H}_{4} \mathrm{CH}_{3}\right), 7.42$ and 7.67 $\left[6 \mathrm{H}\right.$ and $\left.4 \mathrm{H}, 2 \times \mathrm{m}, \mathrm{SiC}\left(\mathrm{CH}_{3}\right)_{3}\left(\mathrm{C}_{6} H_{5}\right)_{2}\right] ; \delta_{\mathrm{C}}\left(125 \mathrm{MHz} ; \mathrm{CDCl}_{3}\right)$ 20.9, 21.5, 21.7 (2 C), and $29.8\left[3 \times \mathrm{COC}_{6} \mathrm{H}_{4} \mathrm{CH}_{3}, \mathrm{COCH}_{3}\right.$, and $\left.\mathrm{CO}\left(\mathrm{CH}_{2}\right)_{2} \mathrm{COCH}_{3}\right], 23.0,28.3,28.7$, and $51.1\left[\mathrm{OCH}_{2}\left(\mathrm{CH}_{2}\right)_{4} \mathrm{~N}_{3}\right]$, $26.8\left[\mathrm{SiC}\left(\mathrm{CH}_{3}\right)_{3}\left(\mathrm{C}_{6} \mathrm{H}_{5}\right)_{2}\right], 27.9$ and $38.0\left[\mathrm{CO}\left(\mathrm{CH}_{2}\right)_{2} \mathrm{COCH}_{3}\right], 52.8$ (C-2), $62.5\left(\mathrm{C}-6^{\prime}\right), 62.8(\mathrm{C}-6), 69.0\left[\mathrm{OCH}_{2}\left(\mathrm{CH}_{2}\right)_{4} \mathrm{~N}_{3}\right], 69.1\left(\mathrm{C}-4^{\prime}\right)$, 69.2 (C-4), 71.8 (C-2'), 72.2 (C-5'), 72.5 (C-3'), 74.8 (C-5), 74.9 $(\mathrm{C}-3), 98.7(\mathrm{C}-1), 101.4\left(\mathrm{C}-1^{\prime}\right), 123.5$ and $133.8\left[\mathrm{~N}(\mathrm{CO})_{2} \mathrm{C}_{6} \mathrm{H}_{4}\right]$, 127.8, 129.7, and $135.8\left[\mathrm{SiC}\left(\mathrm{CH}_{3}\right)_{3}\left(\mathrm{C}_{6} \mathrm{H}_{5}\right)_{2}\right], 128.9(2 \mathrm{C}), 129.2$, $129.8,129.9$, and $130.0\left(\mathrm{COC}_{6} \mathrm{H}_{4} \mathrm{CH}_{3}\right)$; high resolution MALDITOF MS, $m / z$ found $\mathrm{M}+\mathrm{Na} 1337.490, \mathrm{C}_{72} \mathrm{H}_{78} \mathrm{~N}_{4} \mathrm{NaO}_{18}$ Si requires 1337.498 .

\section{5-Azidopentyl (6-O-levulinoyl-2,3,4-tri- $O$-p-toluoyl- $\beta$-D- glucopyranosyl)-(1 $\rightarrow 3)-4-O$-acetyl-2-deoxy-2-phthalimido- $\beta$-D- galactopyranoside 27}

To $26(100 \mathrm{mg}, 76 \mu \mathrm{mol})$ was added a $1 \mathrm{M}$ TBAF solution in THF $\left(5 \mathrm{~cm}^{3}\right)$, before use neutralized at $0{ }^{\circ} \mathrm{C}$ with HOAc. The mixture was stirred for $2 \mathrm{~h}$ at $0{ }^{\circ} \mathrm{C}$ and for $4 \mathrm{~h}$ at rt, when TLC (95:5 $\mathrm{CH}_{2} \mathrm{Cl}_{2}$-acetone) showed the disappearance of $\mathbf{2 6}$ and the formation of a new product $\left(R_{\mathrm{f}}=0.52\right)$. After concentration, a solution of the residue in EtOAc was washed with water and $10 \%$ aq. $\mathrm{NaCl}$, dried, filtered, and concentrated. Column chromatography (95:5 $\mathrm{CH}_{2} \mathrm{Cl}_{2}$-acetone $\rightarrow$ acetone) of the residue 
afforded 27 (74 mg, 91\%), isolated as a white glass; $[\alpha]_{\mathrm{D}}^{20}+2(c 0.3$ in $\left.\mathrm{CHCl}_{3}\right) ; \delta_{\mathrm{H}}\left(500 \mathrm{MHz} ; \mathrm{CDCl}_{3} ; 2 \mathrm{D}\right.$ TOCSY and HSQC) 0.98 , $1.25,1.40$, and 2.85 [each $2 \mathrm{H}, 4 \times \mathrm{m}, \mathrm{OCH}_{2}\left(\mathrm{CH}_{2}\right)_{4} \mathrm{~N}_{3}$ ], 2.13, 2.16, 2.22, 2.24, and 2.26 [each $3 \mathrm{H}, 5 \times \mathrm{s}, 3 \times \mathrm{COC}_{6} \mathrm{H}_{4} \mathrm{CH}_{3}$, $\mathrm{COCH}_{3}$, and $\mathrm{CO}\left(\mathrm{CH}_{2}\right)_{2} \mathrm{COCH}_{3}$ ], 2.54 and 2.70 [each $2 \mathrm{H}$, $2 \times \mathrm{m}, \mathrm{CO}\left(\mathrm{CH}_{2}\right)_{2} \mathrm{COCH}_{3}$ ], 3.23 and 3.70 [each $1 \mathrm{H}, 2 \times \mathrm{m}$, $\mathrm{OCH}_{2}\left(\mathrm{CH}_{2}\right)_{4} \mathrm{~N}_{3}$ ], $3.44\left(1 \mathrm{H}, \mathrm{dd}, J_{\mathrm{H}-5, \mathrm{H}-6 \mathrm{~b}} 8.5 \mathrm{~Hz}, J_{\mathrm{H}-6 \mathrm{a}, \mathrm{H}-6 \mathrm{~b}} 11.8 \mathrm{~Hz}\right.$, $\mathrm{H}-6 \mathrm{~b}), 3.61$ (1 H, dd, $\left.J_{\mathrm{H}-5, \mathrm{H}-6 \mathrm{a}} 4.6, \mathrm{H}-6 \mathrm{a}\right), 3.69(1 \mathrm{H}, \mathrm{m}, \mathrm{H}-5)$, $3.86\left(1 \mathrm{H}, \mathrm{m}, \mathrm{H}-5^{\prime}\right), 4.08\left(1 \mathrm{H}, \mathrm{dd}, J_{\mathrm{H}-5^{\prime}, \mathrm{H}-6 \mathrm{~b}^{\prime}} 5.6, J_{\mathrm{H}-6 \mathrm{a}^{\prime}, \mathrm{H}-6 \mathrm{~b}^{\prime}} 12.1\right.$, $\left.\mathrm{H}-6 \mathrm{~b}^{\prime}\right), 4.22\left(1 \mathrm{H}, \mathrm{dd}, J_{\mathrm{H}-5^{\prime}, \mathrm{H}-6 \mathrm{a}^{\prime}} 2.3, \mathrm{H}-6 \mathrm{a}^{\prime}\right), 4.45$ (1 H, dd, $J_{\mathrm{H}-1, \mathrm{H}-2}$ $\left.8.5, J_{\mathrm{H}-2, \mathrm{H}-3} 11.2, \mathrm{H}-2\right), 4.79\left(1 \mathrm{H}, \mathrm{d}, J_{\mathrm{H}-1^{\prime}, \mathrm{H}-2^{\prime}} 7.8, \mathrm{H}-1^{\prime}\right), 4.81(1 \mathrm{H}$, $\left.\mathrm{dd}, J_{\mathrm{H}-3, \mathrm{H}-4} 3.3, \mathrm{H}-3\right), 4.93(1 \mathrm{H}, \mathrm{d}, \mathrm{H}-1), 5.23\left(1 \mathrm{H}, \mathrm{dd}, J_{\mathrm{H}-2^{\prime}, \mathrm{H}-3^{\prime}}\right.$ 9.8, H-2' $), 5.32(1 \mathrm{H}$, br t, H-4' $), 5.51(1 \mathrm{H}$, br d, H-4), $5.59(1 \mathrm{H}$, br t, $\left.\mathrm{H}-3^{\prime}\right), 6.75,6.90,7.05,7.24,7.48$, and 7.66 (each $2 \mathrm{H}, 6 \times \mathrm{d}$, $\left.3 \times \mathrm{COC}_{6} \mathrm{H}_{4} \mathrm{CH}_{3}\right) ; \delta_{\mathrm{C}}\left(125 \mathrm{MHz} ; \mathrm{CDCl}_{3}\right) 21.0,21.5,21.6(2 \mathrm{C})$, and $29.9\left[3 \times \mathrm{COC}_{6} \mathrm{H}_{4} \mathrm{CH}_{3}, \mathrm{COCH}_{3}\right.$, and $\left.\mathrm{CO}\left(\mathrm{CH}_{2}\right)_{2} \mathrm{COCH}_{3}\right]$, $22.9,28.2,28.7$, and $51.0\left[\mathrm{OCH}_{2}\left(\mathrm{CH}_{2}\right)_{4} \mathrm{~N}_{3}\right], 27.8$ and 38.0 $\left[\mathrm{CO}\left(\mathrm{CH}_{2}\right)_{2} \mathrm{COCH}_{3}\right], 52.6(\mathrm{C}-2), 60.2(\mathrm{C}-6), 62.4\left(\mathrm{C}-6{ }^{\prime}\right), 68.8(\mathrm{C}-$ $\left.4^{\prime}\right), 69.4\left[\mathrm{OCH}_{2}\left(\mathrm{CH}_{2}\right)_{4} \mathrm{~N}_{3}\right], 69.8(\mathrm{C}-4), 71.6\left(\mathrm{C}-2^{\prime}\right), 72.3\left(\mathrm{C}-5^{\prime}\right)$, $72.5\left(\mathrm{C}-3^{\prime}\right), 73.6(\mathrm{C}-5), 75.6(\mathrm{C}-3), 98.9(\mathrm{C}-1), 101.7\left(\mathrm{C}-1^{\prime}\right), 123.4$ and $133.7\left[\mathrm{~N}(\mathrm{CO})_{2} \mathrm{C}_{6} \mathrm{H}_{4}\right], 128.9,129.0,129.3,129.5,129.7$, and $129.9\left(\mathrm{COC}_{6} \mathrm{H}_{4} \mathrm{CH}_{3}\right)$; high resolution MALDI-TOF MS, $\mathrm{m} / \mathrm{z}$ found $\mathrm{M}+\mathrm{Na}$ 1099.376, $\mathrm{C}_{56} \mathrm{H}_{60} \mathrm{~N}_{4} \mathrm{NaO}_{18}$ requires 1099.380.

\section{5-Azidopentyl (6- $O$-levulinoyl-2,3,4-tri- $O$-p-toluoyl- $\beta$-D- glucopyranosyl)-( $\rightarrow 3)-4$,6-di- $O$-acetyl-2-deoxy-2-phthalimido- $\beta$-D-galactopyranoside 28}

A solution of $27(144 \mathrm{mg}, 0.134 \mathrm{mmol})$ in $1: 1$ pyridineacetic anhydride $\left(10 \mathrm{~cm}^{3}\right)$ was stirred overnight, when TLC (95:5 $\mathrm{CH}_{2} \mathrm{Cl}_{2}$-acetone) showed the acetylation to be complete $\left(R_{\mathrm{f}}=0.62\right)$. After co-concentration with toluene, a solution of the residue in $\mathrm{CH}_{2} \mathrm{Cl}_{2}$ was washed with saturated aq. $\mathrm{NaHCO}_{3}$, dried, filtered, and concentrated. Column chromatography (95:5 $\mathrm{CH}_{2} \mathrm{Cl}_{2}$-acetone) of the residue gave $\mathbf{2 8}$ $(125 \mathrm{mg}, 83 \%)$, isolated as a white solid; $[\alpha]_{\mathrm{D}}^{20}+9\left(c 1\right.$ in $\left.\mathrm{CHCl}_{3}\right)$; $\delta_{\mathrm{H}}\left(500 \mathrm{MHz} ; \mathrm{CDCl}_{3} ; 2 \mathrm{D}\right.$ TOCSY and HSQC) $1.00,1.22,1.34$, and 2.81 [each $2 \mathrm{H}, 4 \times \mathrm{m}, \mathrm{OCH}_{2}\left(\mathrm{CH}_{2}\right)_{4} \mathrm{~N}_{3}$ ], 2.01, 2.12, 2.14, and $2.25\left[3 \mathrm{H}, 3 \mathrm{H}, 6 \mathrm{H}\right.$, and $6 \mathrm{H}, 4 \times \mathrm{s}, 3 \times \mathrm{COC}_{6} \mathrm{H}_{4} \mathrm{CH}_{3}$, $2 \times \mathrm{COCH}_{3}$, and $\mathrm{CO}\left(\mathrm{CH}_{2}\right)_{2} \mathrm{COCH}_{3}$ ], 2.55 and 2.72 [each $2 \mathrm{H}, 2 \times \mathrm{m}, \mathrm{CO}\left(\mathrm{CH}_{2}\right)_{2} \mathrm{COCH}_{3}$ ], 3.28 and 3.72 [each $1 \mathrm{H}, 2 \times \mathrm{m}$, $\left.\mathrm{OCH}_{2}\left(\mathrm{CH}_{2}\right)_{4} \mathrm{~N}_{3}\right], 3.84\left(1 \mathrm{H}, \mathrm{m}, \mathrm{H}-5^{\prime}\right), 3.92(1 \mathrm{H}, \mathrm{m}, \mathrm{H}-5), 4.02$ $\left(1 \mathrm{H}, \mathrm{dd}, J_{\mathrm{H}-5, \mathrm{H}-6 \mathrm{~b}} 7.2 \mathrm{~Hz}, J_{\mathrm{H}-6 \mathrm{a}, \mathrm{H}-6 \mathrm{~b}} 11.6 \mathrm{~Hz}, \mathrm{H}-6 \mathrm{~b}\right), 4.07$ (1 H, $\left.\mathrm{dd}, J_{\mathrm{H}-5^{\prime}, \mathrm{H}-6 \mathrm{~b}^{\prime}} 5.2, J_{\mathrm{H}-6 \mathrm{a}^{\prime}, \mathrm{H}-6 \mathrm{~b}^{\prime}} 12.2, \mathrm{H}-6 \mathrm{~b}^{\prime}\right), 4.17\left(1 \mathrm{H}, \mathrm{dd}, J_{\mathrm{H}-5, \mathrm{H}-6 \mathrm{a}}\right.$ 5.1, H-6a), 4.31 (1 H, dd, $\left.J_{\mathrm{H}-5^{\prime}, \mathrm{H}-6 \mathrm{a}^{\prime}} 2.5, \mathrm{H}-6 \mathrm{a}^{\prime}\right), 4.42(1 \mathrm{H}, \mathrm{dd}$, $\left.J_{\mathrm{H}-1, \mathrm{H}-2} 8.7, J_{\mathrm{H}-2, \mathrm{H}-3} 11.3, \mathrm{H}-2\right), 4.71\left(1 \mathrm{H}, \mathrm{d}, J_{\mathrm{H}-1^{\prime}, \mathrm{H}-2^{\prime}} 8.0, \mathrm{H}-1^{\prime}\right)$, $4.76\left(1 \mathrm{H}, \mathrm{dd}, J_{\mathrm{H}-3, \mathrm{H}-4} 3.2, \mathrm{H}-3\right), 4.93(1 \mathrm{H}, \mathrm{d}, \mathrm{H}-1), 5.18(1 \mathrm{H}, \mathrm{dd}$, $\left.J_{\mathrm{H}-2^{\prime}, \mathrm{H}-3^{\prime}} 9.8, \mathrm{H}-2^{\prime}\right), 5.35(1 \mathrm{H}$, br t, H-4' $), 5.53(1 \mathrm{H}$, br d, H-4), $5.57(1 \mathrm{H}$, br t, H-3'), 6.80, 6.91, 7.05, 7.28, 7.48, and 7.66 (each $\left.2 \mathrm{H}, 6 \times \mathrm{d}, 3 \times \mathrm{COC}_{6} \mathrm{H}_{4} \mathrm{CH}_{3}\right) ; \delta_{\mathrm{C}}\left(125 \mathrm{MHz} ; \mathrm{CDCl}_{3}\right) 20.8,21.0$, 21.5, $21.6(2 \mathrm{C})$, and $29.8\left[3 \times \mathrm{COC}_{6} \mathrm{H}_{4} \mathrm{CH}_{3}, 2 \times \mathrm{COCH}_{3}\right.$, and $\left.\mathrm{CO}\left(\mathrm{CH}_{2}\right)_{2} \mathrm{COCH}_{3}\right], 23.0,28.2$, 28.6, and $51.1\left[\mathrm{OCH}_{2}\left(\mathrm{CH}_{2}\right)_{4} \mathrm{~N}_{3}\right]$, 28.0 and $38.0\left[\mathrm{CO}\left(\mathrm{CH}_{2}\right)_{2} \mathrm{COCH}_{3}\right], 52.5(\mathrm{C}-2), 62.2\left(\mathrm{C}-6^{\prime}\right), 62.8$ (C-6), $69.0\left(\mathrm{C}-4^{\prime}\right), 69.2(\mathrm{C}-4), 69.3\left[\mathrm{OCH}_{2}\left(\mathrm{CH}_{2}\right)_{4} \mathrm{~N}_{3}\right], 71.6(\mathrm{C}-$ 5), $71.7\left(\mathrm{C}-2^{\prime}\right), 72.2\left(\mathrm{C}-5^{\prime}\right), 72.5\left(\mathrm{C}-3^{\prime}\right), 74.7$ (C-3), 98.7 (C-1), $101.4\left(\mathrm{C}-1^{\prime}\right), 123.5$ and $133.7\left[\mathrm{~N}(\mathrm{CO})_{2} C_{6} \mathrm{H}_{4}\right], 128.9,129.0,129.2$, 129.6, 129.8, and $129.9\left(\mathrm{COC}_{6} \mathrm{H}_{4} \mathrm{CH}_{3}\right)$; high resolution MALDITOF MS, $m / z$ found $\mathrm{M}+\mathrm{Na} 1141.370, \mathrm{C}_{58} \mathrm{H}_{62} \mathrm{~N}_{4} \mathrm{NaO}_{19}$ requires 1141.390 .

\section{5-Azidopentyl (2,3,4-tri- $O$-p-toluoyl- $\beta$-D-glucopyranosyl)- $(1 \rightarrow 3)-4,6$-di- $O$-acetyl-2-deoxy-2-phthalimido- $\beta$-D- galactopyranoside 29}

To a solution of $28(125 \mathrm{mg}, 0.112 \mathrm{mmol})$ in $\mathrm{EtOH}\left(10 \mathrm{~cm}^{3}\right)$ and toluene $\left(3 \mathrm{~cm}^{3}\right)$ was added hydrazine acetate $(51 \mathrm{mg}, 0.56 \mathrm{mmol})$. The mixture was stirred for $2 \mathrm{~h}$, then concentrated. Column chromatography (95:5 $\mathrm{CH}_{2} \mathrm{Cl}_{2}$-acetone) of the residue yielded 29 $(104 \mathrm{mg}, 91 \%)$, isolated as a white glass; $[a]_{\mathrm{D}}^{20}+2\left(c 1\right.$ in $\left.\mathrm{CHCl}_{3}\right)$; $\delta_{\mathrm{H}}\left(500 \mathrm{MHz} ; \mathrm{CDCl}_{3} ; 2 \mathrm{D}\right.$ TOCSY and HSQC) $0.99,1.20,1.33$, and $2.78\left[\right.$ each $\left.2 \mathrm{H}, 4 \times \mathrm{m}, \mathrm{OCH}_{2}\left(\mathrm{CH}_{2}\right)_{4} \mathrm{~N}_{3}\right], 2.01,2.16,2.22,2.23$, and $2.28\left(\right.$ each $3 \mathrm{H}, 5 \times \mathrm{s}, 3 \times \mathrm{COC}_{6} \mathrm{H}_{4} \mathrm{CH}_{3}$ and $\left.2 \times \mathrm{COCH}_{3}\right), 3.25$ and 3.70 [each $\left.1 \mathrm{H}, 2 \times \mathrm{m}, \mathrm{OCH}_{2}\left(\mathrm{CH}_{2}\right)_{4} \mathrm{~N}_{3}\right], 3.56$ and 3.66 (each 1 $\left.\mathrm{H}, 2 \times \mathrm{m}, 2 \times \mathrm{H}-6^{\prime}\right), 3.72\left(1 \mathrm{H}, \mathrm{m}, \mathrm{H}-5^{\prime}\right), 3.93(1 \mathrm{H}, \mathrm{m}, \mathrm{H}-5), 4.06$ $(2 \mathrm{H}, \mathrm{m}, 2 \times \mathrm{H}-6), 4.44\left(1 \mathrm{H}, \mathrm{dd}, J_{\mathrm{H}-1, \mathrm{H}-2} 8.7 \mathrm{~Hz}, J_{\mathrm{H}-2, \mathrm{H}-3} 11.0 \mathrm{~Hz}\right.$, $\mathrm{H}-2), 4.74\left(1 \mathrm{H}, \mathrm{dd}, J_{\mathrm{H}-3, \mathrm{H}-4} 3.4, \mathrm{H}-3\right), 4.81\left(1 \mathrm{H}, \mathrm{d}, J_{\mathrm{H}-\mathrm{1}^{\prime}, \mathrm{H}-2^{\prime}} 8.0, \mathrm{H}-\right.$ 1'), $4.92(1 \mathrm{H}, \mathrm{d}, \mathrm{H}-1), 5.21$ (1 H, br t, H-2'), $5.27(1 \mathrm{H}$, br t, H-4'), $5.60(1 \mathrm{H}$, br t, H-3'), $5.65(1 \mathrm{H}$, br d, H-4), 6.72, 6.89, 7.06, 7.23, 7.45 , and 7.68 (each $\left.2 \mathrm{H}, 6 \times \mathrm{d}, 3 \times \mathrm{COC}_{6} \mathrm{H}_{4} \mathrm{CH}_{3}\right) ; \delta_{\mathrm{C}}(125 \mathrm{MHz}$; $\left.\mathrm{CDCl}_{3}\right) 20.7,21.3,21.5,21.6$, and $21.7\left(3 \times \mathrm{COC}_{6} \mathrm{H}_{4} \mathrm{CH}_{3}\right.$ and $\left.2 \times \mathrm{COCH}_{3}\right), 22.9,28.3,28.7$, and $51.1\left[\mathrm{OCH}_{2}\left(\mathrm{CH}_{2}\right)_{4} \mathrm{~N}_{3}\right], 52.4$ (C-2), $61.6\left(\mathrm{C}^{\prime} 6^{\prime}\right), 62.0(\mathrm{C}-6), 68.8\left(\mathrm{C}-4^{\prime}\right), 69.3\left[\mathrm{OCH}_{2}\left(\mathrm{CH}_{2}\right)_{4} \mathrm{~N}_{3}\right]$, 69.4 (C-4), 71.2 (C-5), 71.7 (C-2'), $72.6\left(\mathrm{C}-3^{\prime}\right), 75.3\left(\mathrm{C}-5^{\prime}\right), 76.2$ (C-3), 98.8 (C-1), $102.2\left(\mathrm{C}-1 '^{\prime}\right), 123.3$ and 133.6 [N(CO) $\left.{ }_{2} \mathrm{C}_{6} \mathrm{H}_{4}\right]$, $128.9,129.0,129.3,129.5,129.7$, and $130.0\left(\mathrm{COC}_{6} \mathrm{H}_{4} \mathrm{CH}_{3}\right)$; high resolution MALDI-TOF MS, $\mathrm{m} / \mathrm{z}$ found $\mathrm{M}+\mathrm{Na}$ 1043.354, $\mathrm{C}_{53} \mathrm{H}_{56} \mathrm{~N}_{4} \mathrm{NaO}_{17}$ requires 1043.353.

\section{5-Azidopentyl (sodium $\beta$-D-glucopyranosyl 6-sulfate)-(1 $\rightarrow 3)-2$ - acetamido-2-deoxy- $\beta$-D-galactopyranoside 31}

To a solution of $29(95 \mathrm{mg}, 93 \mu \mathrm{mol})$ in $\operatorname{DMF}\left(5 \mathrm{~cm}^{3}\right)$ was added the sulfur trioxide trimethylamine complex $(515 \mathrm{mg}$, $3.67 \mathrm{mmol}$ ). The mixture was stirred for $48 \mathrm{~h}$ at $50{ }^{\circ} \mathrm{C}$, when TLC (9: $1 \mathrm{CH}_{2} \mathrm{Cl}_{2}$-methanol) showed the complete conversion of 29 into non-sodiated $30\left(R_{\mathrm{f}}=0.20\right)$. After quenching the reaction with $\mathrm{MeOH}\left(10 \mathrm{~cm}^{3}\right)$, the solution was co-concentrated with toluene. A solution of the residue in $\mathrm{CH}_{2} \mathrm{Cl}_{2}\left(50 \mathrm{~cm}^{3}\right)$ was washed with saturated aq. $\mathrm{NaHCO}_{3}$, dried, filtered, and concentrated. The residue was dissolved in $\mathrm{MeOH}\left(10 \mathrm{~cm}^{3}\right)$, containing Dowex 50W X $8 \mathrm{Na}^{+}$resin, and stirred for $15 \mathrm{~min}$, then filtered and concentrated. Column chromatography $(95: 5$ $\left.\mathrm{CH}_{2} \mathrm{Cl}_{2}-\mathrm{MeOH}\right)$ of the residue gave $30(52 \mathrm{mg}, 50 \%)$, isolated as a white, amorphous, powder; $\delta_{\mathrm{H}}\left(500 \mathrm{MHz} ; \mathrm{CDCl}_{3}\right) 1.01$, 1.22 , and $2.85\left[2 \mathrm{H}, 4 \mathrm{H}\right.$, and $\left.2 \mathrm{H}, 3 \mathrm{~m}, \mathrm{OCH}_{2}\left(\mathrm{CH}_{2}\right)_{4} \mathrm{~N}_{3}\right], 2.07$, $2.19,2.23,2.32$, and 2.36 (each $3 \mathrm{H}, 5 \times \mathrm{s}, 3 \times \mathrm{COC}_{6} \mathrm{H}_{4} \mathrm{CH}_{3}$ and $\left.2 \times \mathrm{COCH}_{3}\right), 3.33$ and 3.78 [each $1 \mathrm{H}, 2 \times \mathrm{m}, \mathrm{OCH}_{2}\left(\mathrm{CH}_{2}\right)_{4} \mathrm{~N}_{3}$ ], $4.04\left(2 \mathrm{H}, \mathrm{m}, \mathrm{H}-5\right.$ and $\left.\mathrm{H}-5^{\prime}\right), 4.14$ and 4.24 (each $1 \mathrm{H}, 2 \times \mathrm{m}$, $2 \times \mathrm{H}-6), 4.29$ and 4.59 (each $\left.1 \mathrm{H}, 2 \times \mathrm{m}, 2 \times \mathrm{H}-6^{\prime}\right), 4.48(1 \mathrm{H}$, $\left.\mathrm{dd}, J_{\mathrm{H}-1, \mathrm{H}-2} 8.6 \mathrm{~Hz}, J_{\mathrm{H}-2, \mathrm{H}-3} 11.2 \mathrm{~Hz}, \mathrm{H}-2\right), 4.80\left(1 \mathrm{H}, \mathrm{d}, J_{\mathrm{H}-\mathrm{I}^{\prime}, \mathrm{H}-2^{\prime}} 7.6\right.$, H-1' $), 4.91$ (1 H, dd, $\left.J_{\mathrm{H}-3, \mathrm{H}-4} 2.9, \mathrm{H}-3\right), 5.03(1 \mathrm{H}, \mathrm{d}, \mathrm{H}-1), 5.30$ $(1 \mathrm{H}$, br t, H-2' $), 5.50\left(1 \mathrm{H}\right.$, br t, H- $\left.4^{\prime}\right), 5.64\left(1 \mathrm{H}\right.$, br t, $\left.\mathrm{H}-3^{\prime}\right), 5.88$ ( $1 \mathrm{H}$, br d, H-4), 6.85, 6.92, 7.10, 7.35, 7.52, and 7.75 (each $2 \mathrm{H}$, $\left.6 \times \mathrm{d}, 3 \times \mathrm{COC}_{6} \mathrm{H}_{4} \mathrm{CH}_{3}\right)$.

A solution of $30(52 \mathrm{mg}, 46 \mu \mathrm{mol})$ in ethanolic $33 \% \mathrm{CH}_{3} \mathrm{NH}_{2}$ $\left(5 \mathrm{~cm}^{3}\right)$ was stirred for 7 days, during which time the mixture was three times concentrated and fresh ethanolic $33 \% \mathrm{CH}_{3} \mathrm{NH}_{2}$ $\left(5 \mathrm{~cm}^{3}\right)$ was added. After co-concentration with toluene, to a solution of the residue in dry $\mathrm{MeOH}$ at $0{ }^{\circ} \mathrm{C}$ was added acetic anhydride $\left(100 \mathrm{~mm}^{3}\right)$. The mixture was stirred for $3 \mathrm{~h}$ at $0{ }^{\circ} \mathrm{C}$, then concentrated. Size-exclusion chromatography (Bio-Gel P2, $\left.100 \mathrm{mM} \mathrm{NH}_{4} \mathrm{HCO}_{3}\right)$ of the residue afforded $31(21 \mathrm{mg}, 76 \%)$, isolated after lyophilization from water, as a white, amorphous powder; $[a]_{\mathrm{D}}^{20}-14$ ( $c 1$ in water $) ; \delta_{\mathrm{H}}\left(500 \mathrm{MHz} ; \mathrm{D}_{2} \mathrm{O} ; 2 \mathrm{D}\right.$ TOCSY and HSQC) $1.40,1.59$, and $3.33[2 \mathrm{H}, 4 \mathrm{H}$, and $2 \mathrm{H}, 3 \times \mathrm{m}$, $\mathrm{OCH}_{2}\left(\mathrm{CH}_{2}\right)_{4} \mathrm{~N}_{3}$ ], $2.03(3 \mathrm{H}, \mathrm{s}, \mathrm{NAc}), 3.31\left(1 \mathrm{H}\right.$, br t, $\left.\mathrm{H}-2^{\prime}\right), 3.45$ $(1 \mathrm{H}$, br t, H-4' $), 3.46(1 \mathrm{H}$, br t, H-3'), 3.61 and 3.92 [each $1 \mathrm{H}$, $\left.2 \times \mathrm{m}, \mathrm{OCH}_{2}\left(\mathrm{CH}_{2}\right)_{4} \mathrm{~N}_{3}\right], 3.62\left(1 \mathrm{H}, \mathrm{m}, \mathrm{H}-5^{\prime}\right), 3.68(1 \mathrm{H}, \mathrm{m}, \mathrm{H}-5)$, $3.78(2 \mathrm{H}, \mathrm{m}, 2 \times \mathrm{H}-6), 3.83\left(1 \mathrm{H}, \mathrm{dd}, J_{\mathrm{H}-2, \mathrm{H}-3} 11.0 \mathrm{~Hz}, J_{\mathrm{H}-3, \mathrm{H}-4} 3.1\right.$ $\mathrm{Hz}, \mathrm{H}-3), 3.99$ (1 H, br t, H-2), 4.18 (1 H, br d, H-4), 4.19 and 4.30 (each $\left.1 \mathrm{H}, 2 \times \mathrm{m}, 2 \times \mathrm{H}-6^{\prime}\right), 4.48\left(1 \mathrm{H}, \mathrm{d}, J_{\mathrm{H}-1, \mathrm{H}-2} 8.9, \mathrm{H}-1\right), 4.50(1$ $\left.\mathrm{H}, \mathrm{d}, J_{\mathrm{H}-1^{\prime}, \mathrm{H}-2^{\prime}} 8.1, \mathrm{H}-1^{\prime}\right) ; \delta_{\mathrm{C}}\left(125 \mathrm{MHz} ; \mathrm{D}_{2} \mathrm{O}\right) 22.9\left(\mathrm{NDCOCH}_{3}\right)$, 23.2, 28.3, 28.8, and 51.8 $\left[\mathrm{OCH}_{2}\left(\mathrm{CH}_{2}\right)_{4} \mathrm{~N}_{3}\right], 51.9(\mathrm{C}-2), 61.8(\mathrm{C}-6)$, $67.9\left(\mathrm{C}-6^{\prime}\right), 68.7$ (C-4), $70.0\left(\mathrm{C}-3^{\prime}\right), 70.8\left[\mathrm{OCH}_{2}\left(\mathrm{CH}_{2}\right)_{4} \mathrm{~N}_{3}\right], 73.5$ (C-2'), $74.2\left(\mathrm{C}-5^{\prime}\right), 75.7$ (C-5), 76.1 (C-4'), 81.0 (C-3), 102.1 (C1), $105.1\left(\mathrm{C}-1^{\prime}\right)$; high resolution MALDI-TOF MS, $\mathrm{m} / \mathrm{z}$ found $\mathrm{M}+\mathrm{Na} 616.157, \mathrm{C}_{19} \mathrm{H}_{33} \mathrm{~N}_{4} \mathrm{Na}_{2} \mathrm{O}_{14} \mathrm{~S}$ requires 616.151.

5-Aminopentyl (sodium $\beta$-D-glucopyranosyl 6-sulfate)-( $1 \rightarrow 3$ )-2acetamido-2-deoxy- $\beta$-D-galactopyranoside 4

A solution of $31(8.4 \mathrm{mg}, 14 \mu \mathrm{mol})$ in $0.05 \mathrm{M}$ aq. $\mathrm{NaOH}$ $\left(1.0 \mathrm{~cm}^{3}\right)$ was added dropwise to a suspension of $10 \% \mathrm{Pd}-\mathrm{C}$ $(2 \mathrm{mg})$ and $\mathrm{NaHB}_{4}(8.0 \mathrm{mg})$ in water $\left(0.5 \mathrm{~cm}^{3}\right)$. The suspension 
was stirred for $45 \mathrm{~min}$, when TLC $(6: 2.5: 1.5 \mathrm{EtOAc}-\mathrm{MeOH}-$ water) showed the disappearance of $\mathbf{3 1}$. After filtration through Celite, size-exclusion chromatography (Bio-Gel P-2, $100 \mathrm{mM}$ $\left.\mathrm{NH}_{4} \mathrm{HCO}_{3}\right)$ gave $4(5.7 \mathrm{mg}, 71 \%)$, isolated after lyophilization from water, as a white, amorphous powder; $[\alpha]_{\mathrm{D}}^{20}-11(c 0.4$ in water $) ; \delta_{\mathrm{H}}\left(500 \mathrm{MHz} ; \mathrm{D}_{2} \mathrm{O} ; 2 \mathrm{D}\right.$ TOCSY and HSQC) $1.42,1.61$, 1.69, and 3.01 [each $2 \mathrm{H}, 4 \times \mathrm{m}, \mathrm{OCH}_{2}\left(\mathrm{CH}_{2}\right)_{4} \mathrm{ND}_{2}$ ], $2.03(3 \mathrm{H}$, s, NAc), $3.34(1 \mathrm{H}$, br t, H-2' $), 3.46(1 \mathrm{H}$, br t, H-4'), $3.47(1 \mathrm{H}$, br t, $\left.\mathrm{H}^{\prime} 3^{\prime}\right), 3.62$ and 3.92 [each $1 \mathrm{H}, 2 \times \mathrm{m}, \mathrm{OCH}_{2}\left(\mathrm{CH}_{2}\right)_{4} \mathrm{ND}_{2}$ ], $3.65\left(1 \mathrm{H}, \mathrm{m}, \mathrm{H}-5^{\prime}\right), 3.68(1 \mathrm{H}, \mathrm{m}, \mathrm{H}-5), 3.79(2 \mathrm{H}, \mathrm{m}, 2 \times \mathrm{H}-$ 6), $3.84\left(1 \mathrm{H}, \mathrm{dd}, J_{\mathrm{H}-2, \mathrm{H}-3} 11.0 \mathrm{~Hz}, J_{\mathrm{H}-3, \mathrm{H}-4} 3.2 \mathrm{~Hz}, \mathrm{H}-3\right), 4.00$ $\left(1 \mathrm{H}, \mathrm{dd}, J_{\mathrm{H}-1, \mathrm{H}-2} 8.6, \mathrm{H}-2\right), 4.18\left(1 \mathrm{H}, \mathrm{dd}, J_{\mathrm{H}-5^{\prime}, \mathrm{H}-6 \mathrm{~b}^{\prime}} 6.2, J_{\mathrm{H}-6 \mathrm{a}^{\prime}, \mathrm{H}-6 \mathrm{~b}^{\prime}}\right.$ 11.2, H-6b' $), 19\left(1 \mathrm{H}\right.$, br d, H-4), $4.32\left(1 \mathrm{H}, \mathrm{dd}, J_{\mathrm{H}-5^{\prime}, \mathrm{H}-6 \mathrm{a}^{\prime}} 2.2\right.$, $\left.\mathrm{H}-6 \mathrm{a}^{\prime}\right), 4.49$ (1 H, d, H-1), $4.51\left(1 \mathrm{H}, \mathrm{d}, J_{\mathrm{H}-1^{\prime}, \mathrm{H}-2^{\prime}} 8.0, \mathrm{H}-1^{\prime}\right)$; $\delta_{\mathrm{C}}\left(125 \mathrm{MHz} ; \mathrm{D}_{2} \mathrm{O}\right) 22.8\left(\mathrm{NDCOCH}_{3}\right), 22.8,27.0,28.7$, and 40.0 $\left[\mathrm{OCH}_{2}\left(\mathrm{CH}_{2}\right)_{4} \mathrm{ND}_{2}\right], 52.0(\mathrm{C}-2), 61.9(\mathrm{C}-6), 68.0\left(\mathrm{C}-6^{\prime}\right), 68.6(\mathrm{C}-$ 4), $70.0\left(\mathrm{C}-3^{\prime}\right), 70.3\left[\mathrm{OCH}_{2}\left(\mathrm{CH}_{2}\right)_{4} \mathrm{ND}_{2}\right], 73.4\left(\mathrm{C}-2^{\prime}\right), 74.3\left(\mathrm{C}-5^{\prime}\right)$, 75.7 (C-5), 76.2 (C-4'), 80.9 (C-3), 102.2 (C-1), 105.0 (C-1'); high resolution MALDI-TOF MS, $m / z$ found $\mathrm{M}+\mathrm{Na} 593.166$, $\mathrm{C}_{19} \mathrm{H}_{35} \mathrm{~N}_{2} \mathrm{Na}_{2} \mathrm{O}_{14} \mathrm{~S}$ requires 593.160.

\section{5-Azidopentyl (3,4,6-tri- $O$-acetyl-2-deoxy-2-phthalimido- $\beta$-D-galactopyranosyl)-(1 $\rightarrow 6)-[(6-O$-levulinoyl- 2,3,4-tri- $O$-p-toluoyl- $\beta$-D-glucopyranosyl)- $(1 \rightarrow$ 3)]-4-O-acetyl-2-deoxy-2-phthalimido- $\beta$-D-galactopyranoside 33}

A solution of 27 ( $92 \mathrm{mg}, 86 \mu \mathrm{mol})$ and 3,4,6-tri- $O$-acetyl-2-deoxy2 -phthalimido- $\beta$-D-galactopyranosyl trichloroacetimidate ${ }^{28}$ (32; $74.3 \mathrm{mg}, 128 \mu \mathrm{mol})$ in dry $\mathrm{CH}_{2} \mathrm{Cl}_{2}\left(5 \mathrm{~cm}^{3}\right)$, containing activated molecular sieves ( $4 \AA, 0.5 \mathrm{~g}$ ), was stirred for $30 \mathrm{~min}$ at $\mathrm{rt}$, then TMSOTf $\left(2.3 \mathrm{~mm}^{3}, 12.8 \mu \mathrm{mol}\right)$ was added at $0{ }^{\circ} \mathrm{C}$. The mixture was stirred for $15 \mathrm{~min}$ at $0{ }^{\circ} \mathrm{C}$, when TLC $\left(9: 1 \mathrm{CH}_{2} \mathrm{Cl}_{2}\right.$-acetone $)$ showed the formation of $33\left(R_{\mathrm{f}}=0.50\right)$. After neutralization with pyridine and filtration, the solution was washed with $10 \%$ aq. $\mathrm{NaCl}$, dried, filtered, and concentrated. Column chromatography $\left(9: 1 \mathrm{CH}_{2} \mathrm{Cl}_{2}\right.$-acetone) of the residue rendered $33(74 \mathrm{mg}, 58 \%)$, isolated as a glass; $[a]_{\mathrm{D}}^{20}+2\left(c 1\right.$ in $\left.\mathrm{CHCl}_{3}\right)$; $\delta_{\mathrm{H}}\left(500 \mathrm{MHz} ; \mathrm{CDCl}_{3} ; 2 \mathrm{D}\right.$ TOCSY and HSQC) $0.83,1.00,1.12$, and 2.72 [each $2 \mathrm{H}, 4 \times \mathrm{m}, \mathrm{OCH}_{2}\left(\mathrm{CH}_{2}\right)_{4} \mathrm{~N}_{3}$ ] $, 1.76,2.02,2.11$, $2.13,2.14,2.16,2.25$, and 2.26 [each $3 \mathrm{H}, 8 \times \mathrm{s}, 3 \times \mathrm{COC}_{6} \mathrm{H}_{4} \mathrm{CH}_{3}$, $4 \times \mathrm{COCH}_{3}, \mathrm{CO}\left(\mathrm{CH}_{2}\right)_{2} \mathrm{COCH}_{3}$ ], 2.51 and 2.68 [each $2 \mathrm{H}$, $2 \times \mathrm{m}, \mathrm{CO}\left(\mathrm{CH}_{2}\right)_{2} \mathrm{COCH}_{3}$ ], 2.94 and 3.25 [each $1 \mathrm{H}, 2 \times \mathrm{m}$, $\left.\mathrm{OCH}_{2}\left(\mathrm{CH}_{2}\right)_{4} \mathrm{~N}_{3}\right], 3.51\left(1 \mathrm{H}, \mathrm{dd}, J_{\mathrm{H}-5, \mathrm{H}-6 \mathrm{~b}} 8.6 \mathrm{~Hz}, J_{\mathrm{H}-6 \mathrm{a}, \mathrm{H}-6 \mathrm{~b}} 10.9 \mathrm{~Hz}\right.$, H-6b), 3.77 (2 H, m, H-5 and H-5"), 3.90 (1 H, dd, $J_{\mathrm{H}-5, \mathrm{H}-6 \mathrm{a}} 2.0$, H-6a), 4.02 (1 H, br t, H-5'), 4.08 (1 H, dd, $J_{\mathrm{H}-5^{\prime \prime}, \mathrm{H}-6 \mathrm{~b}^{\prime \prime}} 4.9, J_{\mathrm{H}-6 \mathrm{a}^{\prime \prime}, \mathrm{H}-6 \mathrm{~b}^{\prime \prime}}$ $\left.12.2, \mathrm{H}-6 \mathrm{~b}^{\prime \prime}\right), 4.14\left(2 \mathrm{H}, \mathrm{m}, 2 \times \mathrm{H}-6^{\prime}\right), 4.19\left(1 \mathrm{H}, \mathrm{dd}, J_{\mathrm{H}-5^{\prime \prime}, \mathrm{H}-6 \mathrm{a}^{\prime \prime}} 2.8\right.$, $\left.\mathrm{H}-6 \mathrm{a}^{\prime \prime}\right), 4.30\left(1 \mathrm{H}, \mathrm{dd}, J_{\mathrm{H}-1, \mathrm{H}-2} 8.6, J_{\mathrm{H}-2, \mathrm{H}-3} 11.3, \mathrm{H}-2\right), 4.44(1 \mathrm{H}$, $\left.\mathrm{dd}, J_{\mathrm{H}-1^{\prime}, \mathrm{H}-2^{\prime}} 8.4, J_{\mathrm{H}-2^{\prime}, \mathrm{H}-3^{\prime}} 11.4, \mathrm{H}-2^{\prime}\right), 4.63\left(1 \mathrm{H}, \mathrm{dd}, J_{\mathrm{H}-3, \mathrm{H}-4} 3.4\right.$, $\mathrm{H}-3), 4.65$ (1 H, d, $\left.J_{\mathrm{H}-1^{\prime \prime}, \mathrm{H}-2^{\prime \prime}} 8.0, \mathrm{H}-1^{\prime \prime}\right), 4.75(1 \mathrm{H}, \mathrm{d}, \mathrm{H}-1), 5.15$ $\left(1 \mathrm{H}, \mathrm{dd}, J_{\mathrm{H}-2^{\prime \prime}, \mathrm{H}-3^{\prime \prime}} 9.8, \mathrm{H}-2^{\prime \prime}\right), 5.28\left(1 \mathrm{H}, \mathrm{d}, \mathrm{H}-1^{\prime}\right), 5.32(1 \mathrm{H}, \mathrm{br} \mathrm{t}$, $\left.\mathrm{H}-4^{\prime \prime}\right), 5.37$ (1 H, br d, H-4), $5.40\left(1 \mathrm{H}\right.$, br d, $\left.J_{\mathrm{H}-3^{\prime}, \mathrm{H}-4^{\prime}} 3.4, \mathrm{H}-4^{\prime}\right)$, $5.53\left(1 \mathrm{H}\right.$, br t, H-3"), $5.72\left(1 \mathrm{H}, \mathrm{dd}, \mathrm{H}-3^{\prime}\right), 6.78,6.90,7.05,7.24$, 7.46, and 7.65 (each $2 \mathrm{H}, 6 \times \mathrm{d}, 3 \times \mathrm{COC}_{6} \mathrm{H}_{4} \mathrm{CH}_{3}$ ), 7.41 and 7.72 (each $4 \mathrm{H}, 2 \times \mathrm{m}, 2 \times \mathrm{Phth}) ; \delta_{\mathrm{C}}\left(125 \mathrm{MHz} ; \mathrm{CDCl}_{3}\right) 19.5$, 19.6, 19.7, 19.8, 19.9, 20.5, 20.6, and $29.0\left[3 \times \mathrm{COC}_{6} \mathrm{H}_{4} \mathrm{CH}_{3}\right.$, $4 \times \mathrm{COCH}_{3}$, and $\left.\mathrm{CO}\left(\mathrm{CH}_{2}\right)_{2} \mathrm{COCH}_{3}\right], 21.9,27.1,27.6$, and 50.0 $\left[\mathrm{OCH}_{2}\left(\mathrm{CH}_{2}\right)_{4} \mathrm{~N}_{3}\right], 26.9$ and $36.9\left[\mathrm{CO}\left(\mathrm{CH}_{2}\right)_{2} \mathrm{COCH}_{3}\right], 50.4\left(\mathrm{C}-2^{\prime}\right)$, $51.4(\mathrm{C}-2), 60.2\left(\mathrm{C}-6^{\prime}\right), 61.2\left(\mathrm{C}-6^{\prime \prime}\right), 65.7\left(\mathrm{C}-4^{\prime}\right), 66.9\left(\mathrm{C}-3^{\prime}\right), 67.6$ $\left[\mathrm{OCH}_{2}\left(\mathrm{CH}_{2}\right)_{4} \mathrm{~N}_{3}\right], 67.7\left(\mathrm{C}-4^{\prime \prime}\right), 67.8(\mathrm{C}-6), 68.7(\mathrm{C}-4), 69.8\left(\mathrm{C}-5^{\prime}\right)$, $70.6\left(\mathrm{C}-2^{\prime \prime}\right), 71.2$ and $72.7\left(\mathrm{C}-5\right.$ and C-5"), $71.4\left(\mathrm{C}-3^{\prime \prime}\right), 73.4(\mathrm{C}-$ 3), $97.3\left(\mathrm{C}-1^{\prime}\right), 97.2(\mathrm{C}-1), 100.3\left(\mathrm{C}-1^{\prime \prime}\right), 121.8,122.4,132.7$, and $133.3\left[2 \times \mathrm{N}(\mathrm{CO})_{2} \mathrm{C}_{6} \mathrm{H}_{4}\right], 127.8,127.9,128.2,128.5,128.7$, and $128.9\left(\mathrm{COC}_{6} \mathrm{H}_{4} \mathrm{OCH}_{3}\right)$; high resolution MALDI-TOF MS, $\mathrm{m} / \mathrm{z}$ found $\mathrm{M}+\mathrm{Na} 1516.456, \mathrm{C}_{76} \mathrm{H}_{79} \mathrm{~N}_{5} \mathrm{NaO}_{27}$ requires 1516.486 .

\section{5-Azidopentyl (3,4,6-tri- $O$-acetyl-2-deoxy- 2-phthalimido- $\beta$-D-galactopyranosyl $)-(1 \rightarrow$ \\ 6)-[(2,3,4-tri- $O$ - $p$-toluoyl- $\beta$-D-glucopyranosyl)-(1 $\rightarrow 3)]-4-O$ - acetyl-2-deoxy-2-phthalimido- $\beta$-D-galactopyranoside 34}

To a solution of $33(70 \mathrm{mg}, 47 \mu \mathrm{mol})$ in $\operatorname{EtOH}\left(5 \mathrm{~cm}^{3}\right)$ and toluene $\left(1.5 \mathrm{~cm}^{3}\right)$ was added hydrazine acetate $(21.3 \mathrm{mg}$,
$230 \mu \mathrm{mol})$. The mixture was stirred for $2 \mathrm{~h}$, then concentrated. Column chromatography $\left(9: 1 \mathrm{CH}_{2} \mathrm{Cl}_{2}\right.$-acetone) of the residue yielded $34(48 \mathrm{mg}, 74 \%)$, isolated as a glass; $[\alpha]_{\mathrm{D}}^{20}+5\left(c 1 \mathrm{in} \mathrm{CHCl}_{3}\right)$; $\delta_{\mathrm{H}}\left(500 \mathrm{MHz} ; \mathrm{CDCl}_{3} ; 2 \mathrm{D}\right.$ TOCSY and HSQC) $0.81,1.09,1.11$, and 2.69 [each $2 \mathrm{H}, 4 \times \mathrm{m}, \mathrm{OCH}_{2}\left(\mathrm{CH}_{2}\right)_{4} \mathrm{~N}_{3}$ ] $, 1.77,2.01,2.14$, $2.15,2.18,2.21$, and 2.28 (each $3 \mathrm{H}, 7 \times \mathrm{s}, 3 \times \mathrm{COC}_{6} \mathrm{H}_{4} \mathrm{CH}_{3}$ and $\left.4 \times \mathrm{COCH}_{3}\right), 2.96$ and 3.29 [each $1 \mathrm{H}, 2 \times \mathrm{m}, \mathrm{OCH}_{2}\left(\mathrm{CH}_{2}\right)_{4} \mathrm{~N}_{3}$ ], $3.49(1 \mathrm{H}, \mathrm{m}, \mathrm{H}-6 \mathrm{~b}), 3.50$ and 3.65 (each $\left.1 \mathrm{H}, 2 \times \mathrm{m}, 2 \times \mathrm{H}-6^{\prime \prime}\right)$, $3.66\left(1 \mathrm{H}, \mathrm{m}, \mathrm{H}-5^{\prime \prime}\right), 3.77$ (1 H, m, H-5), 3.88 (1 H, dd, $J_{\mathrm{H}-5, \mathrm{H}-6 \mathrm{a}}$ $\left.2.6 \mathrm{~Hz}, J_{\mathrm{H}-6 \mathrm{a}, \mathrm{H}-6 \mathrm{~b}} 10.7 \mathrm{~Hz}, \mathrm{H}-6 \mathrm{a}\right), 4.01\left(1 \mathrm{H}, \mathrm{m}, \mathrm{H}-5^{\prime}\right), 4.14(2 \mathrm{H}$, $\left.\mathrm{m}, 2 \times \mathrm{H}^{-6} 6^{\prime}\right), 4.31\left(1 \mathrm{H}, \mathrm{dd}, J_{\mathrm{H}-1, \mathrm{H}-2} 8.6, J_{\mathrm{H}-2, \mathrm{H}-3} 11.2, \mathrm{H}-2\right), 4.44$ $\left(1 \mathrm{H}, \mathrm{dd}, J_{\mathrm{H}-1^{\prime}, \mathrm{H}-2^{\prime}} 8.4, J_{\mathrm{H}-2^{\prime}, \mathrm{H}-3^{\prime}} 11.5, \mathrm{H}-2^{\prime}\right), 4.63\left(1 \mathrm{H}, \mathrm{dd}, J_{\mathrm{H}-3, \mathrm{H}-4}\right.$ 3.4, H-3), $4.73\left(1 \mathrm{H}, \mathrm{d}, J_{\mathrm{H}-1^{\prime \prime} \mathrm{H}-2^{\prime \prime}} 8.3, \mathrm{H}-1^{\prime \prime}\right), 4.75(1 \mathrm{H}, \mathrm{d}, \mathrm{H}-1)$, $5.16\left(1 \mathrm{H}, \mathrm{dd}, J_{\mathrm{H}-2^{\prime \prime}, \mathrm{H}-3^{\prime \prime}} 10.0, \mathrm{H}-2^{\prime \prime}\right), 5.25(1 \mathrm{H}$, br t, H-4"), 5.28 $\left(1 \mathrm{H}, \mathrm{d}, \mathrm{H}-1^{\prime}\right), 5.39\left(1 \mathrm{H}\right.$, br d, $\left.J_{\mathrm{H}-3^{\prime}, \mathrm{H}-4^{\prime}} 3.1, \mathrm{H}-4^{\prime}\right), 5.51(1 \mathrm{H}, \mathrm{br}$ d, H-4), $5.57\left(1 \mathrm{H}\right.$, br t, H-3"), $5.71\left(1 \mathrm{H}, \mathrm{dd}, \mathrm{H}-3^{\prime}\right), 6.70,6.89$, 7.06, 7.21, 7.44, and 7.68 (each $2 \mathrm{H}, 6 \times \mathrm{d}, 3 \times \mathrm{COC}_{6} \mathrm{H}_{4} \mathrm{CH}_{3}$ ), 7.30 and 7.75 (each $4 \mathrm{H}, 2 \times \mathrm{m}, 2 \times \mathrm{Phth}) ; \delta_{\mathrm{C}}\left(125 \mathrm{MHz} ; \mathrm{CDCl}_{3}\right)$ $19.4,19.7,19.8,20.2,20.4,20.5$, and $20.6\left(3 \times \mathrm{COC}_{6} \mathrm{H}_{4} \mathrm{CH}_{3}\right.$ and $\left.4 \times \mathrm{COCH}_{3}\right), 21.9,27.2,28.6$, and $50.0\left[\mathrm{OCH}_{2}\left(\mathrm{CH}_{2}\right)_{4} \mathrm{~N}_{3}\right], 50.3$ $\left(\mathrm{C}-2^{\prime}\right), 51.4(\mathrm{C}-2), 60.2\left(\mathrm{C}-6^{\prime \prime}\right), 60.4\left(\mathrm{C}-6^{\prime}\right), 65.7\left(\mathrm{C}-4^{\prime}\right), 66.9$ (C6), $67.0\left(\mathrm{C}-3^{\prime}\right), 67.6\left(\mathrm{C}-4^{\prime \prime}\right), 67.7\left[\mathrm{OCH}_{2}\left(\mathrm{CH}_{2}\right)_{4} \mathrm{~N}_{3}\right], 68.9(\mathrm{C}-4)$, $69.9\left(\mathrm{C}-5^{\prime}\right), 70.6\left(\mathrm{C}-2^{\prime \prime}\right), 71.5\left(\mathrm{C}-3^{\prime \prime}\right), 72.3(\mathrm{C}-5), 74.3\left(\mathrm{C}-5^{\prime \prime}\right), 74.9$ (C-3), 97.0 (C-1'), 97.3 (C-1), 101.0 (C-1"), 121.6, 122.6, 132.4, and $133.3\left[2 \times \mathrm{N}(\mathrm{CO})_{2} \mathrm{C}_{6} \mathrm{H}_{4}\right], 127.8,127.9,128.1,128.4,128.7$, and $128.9\left(\mathrm{COC}_{6} \mathrm{H}_{4} \mathrm{OCH}_{3}\right)$; high resolution MALDI-TOF MS, $m / z$ found $\mathrm{M}+\mathrm{Na} 1418.415, \mathrm{C}_{71} \mathrm{H}_{73} \mathrm{~N}_{5} \mathrm{NaO}_{25}$ requires 1418.449 .

\section{5-Azidopentyl (2-acetamido-2-deoxy- $\beta$-D-galactopyranosyl)- $(1 \rightarrow 6)$-[(sodium $\beta$-D-glucopyranosyl 6-sulfate)-( $1 \rightarrow 3)]-2-$ acetamido-2-deoxy- $\beta$-D-galactopyranoside 36}

To a solution of 34 (43 mg, $30.8 \mu \mathrm{mol})$ in DMF $\left(3 \mathrm{~cm}^{3}\right)$ was added the sulfur trioxide trimethylamine complex (173 $\mathrm{mg}$, $1.22 \mathrm{mmol})$. The mixture was stirred for $48 \mathrm{~h}$ at $50{ }^{\circ} \mathrm{C}$, when TLC (9: $\left.1 \mathrm{CH}_{2} \mathrm{Cl}_{2}-\mathrm{MeOH}\right)$ showed the complete conversion of 34 into non-sodiated $35\left(R_{\mathrm{f}}=0.38\right)$. After quenching the reaction with $\mathrm{MeOH}\left(10 \mathrm{~cm}^{3}\right)$, the solution was co-concentrated with toluene. A solution of the residue in $\operatorname{EtOAc}\left(50 \mathrm{~cm}^{3}\right)$ was washed with saturated aq. $\mathrm{NaHCO}_{3}$ and $10 \%$ aq. $\mathrm{NaCl}$, dried, filtered, and concentrated. The residue was dissolved in $\mathrm{MeOH}\left(10 \mathrm{~cm}^{3}\right)$, containing Dowex 50W X $8 \mathrm{Na}^{+}$resin, and stirred for $15 \mathrm{~min}$, then filtered and concentrated. Column chromatography $(9: 1$ $\left.\mathrm{CH}_{2} \mathrm{Cl}_{2}-\mathrm{MeOH}\right)$ of the residue gave $35(38 \mathrm{mg}, 82 \%)$, isolated as a white, amorphous powder; $\delta_{\mathrm{H}}\left(300 \mathrm{MHz} ; \mathrm{CDCl}_{3}\right) 0.90,1.20$, and $2.80\left[2 \mathrm{H}, 4 \mathrm{H}\right.$, and $\left.2 \mathrm{H}, 3 \times \mathrm{m}, \mathrm{OCH}_{2}\left(\mathrm{CH}_{2}\right)_{4} \mathrm{~N}_{3}\right], 1.83,2.05,2.17$, 2.26, 2.33, 2.34, and 2.38 (each $3 \mathrm{H}, 7 \times \mathrm{s}, 3 \times \mathrm{COC}_{6} \mathrm{H}_{4} \mathrm{CH}_{3}$ and $\left.4 \times \mathrm{COCH}_{3}\right), 3.19$ and 3.50 [each $1 \mathrm{H}, 2 \times \mathrm{m}, \mathrm{OCH}_{2}\left(\mathrm{CH}_{2}\right)_{4} \mathrm{~N}_{3}$ ], 3.51 and 3.99 (each $1 \mathrm{H}, 2 \times \mathrm{m}, 2 \times \mathrm{H}-6), 3.78(1 \mathrm{H}, \mathrm{m}, \mathrm{H}-5)$, $4.17\left(2 \mathrm{H}, \mathrm{m}, 2 \times \mathrm{H}-6^{\prime \prime}\right), 4.36\left(2 \mathrm{H}, \mathrm{m}, 2 \times \mathrm{H}-6^{\prime}\right), 4.47(1 \mathrm{H}, \mathrm{dd}$, $\left.J_{\mathrm{H}-1^{\prime}, \mathrm{H}-2^{\prime}} 8.4 \mathrm{~Hz}, J_{\mathrm{H}-2^{\prime}, \mathrm{H}-3^{\prime}} 11.4 \mathrm{~Hz}, \mathrm{H}-2^{\prime}\right), 4.73\left(1 \mathrm{H}, \mathrm{d}, J_{\mathrm{H}-1^{\prime \prime}, \mathrm{H}-2^{\prime \prime}} 7.7\right.$, $\left.\mathrm{H}-1^{\prime \prime}\right), 4.81\left(1 \mathrm{H}, \mathrm{dd}, J_{\mathrm{H}-2, \mathrm{H}-3} 11.3, J_{\mathrm{H}-3, \mathrm{H}-4} 2.8, \mathrm{H}-3\right), 4.87(1 \mathrm{H}, \mathrm{d}$, $\left.J_{\mathrm{H}-1, \mathrm{H}-2} 8.5, \mathrm{H}-1\right), 5.26\left(1 \mathrm{H}, \mathrm{d}, \mathrm{H}-1^{\prime}\right), 5.38\left(1 \mathrm{H}, \mathrm{br} \mathrm{d}, \mathrm{H}-4^{\prime}\right), 5.55$ (1 H, br t, H-4"), $5.56\left(1 \mathrm{H}\right.$, br t, H-3"), $5.77\left(1 \mathrm{H}, \mathrm{dd}, J_{\mathrm{H}-3^{\prime}, \mathrm{H}-4^{\prime}}\right.$ $\left.3.3, \mathrm{H}-3^{\prime}\right), 5.83$ (1 H, br d, H-4), 6.87, 6.99, 7.19, 7.35, 7.49, and 7.72 (each $\left.2 \mathrm{H}, 6 \times \mathrm{d}, 3 \times \mathrm{COC}_{6} \mathrm{H}_{4} \mathrm{CH}_{3}\right), 7.46$ and 7.76 (each $4 \mathrm{H}, 2 \times \mathrm{m}, 2 \times \mathrm{Phth})$.

A solution of $36(33 \mathrm{mg}, 22 \mu \mathrm{mol})$ in ethanolic $33 \% \mathrm{CH}_{3} \mathrm{NH}_{2}$ $\left(5 \mathrm{~cm}^{3}\right)$ was stirred for 7 days, during which time the mixture was three times concentrated and fresh ethanolic $33 \% \mathrm{CH}_{3} \mathrm{NH}_{2}$ $\left(5 \mathrm{~cm}^{3}\right)$ was added. After co-concentration with toluene, to a solution of the residue in dry $\mathrm{MeOH}$ at $0{ }^{\circ} \mathrm{C}$ was added acetic anhydride $\left(100 \mathrm{~mm}^{3}\right)$. The mixture was stirred for $3 \mathrm{~h}$ at $0{ }^{\circ} \mathrm{C}$, then concentrated. Size-exclusion chromatography (Bio-Gel $\left.\mathrm{P}-2,100 \mathrm{mM} \mathrm{NH} \mathrm{HCO}_{3}\right)$ of the residue afforded $36(13 \mathrm{mg}$, $74 \%$ ), isolated after lyophilization from water, as a white, amorphous powder; $[\alpha]_{\mathrm{D}}^{20}+6(c 0.2$ in water $) ; \delta_{\mathrm{H}}\left(500 \mathrm{MHz} ; \mathrm{D}_{2} \mathrm{O}\right.$; 2D TOCSY and HSQC) 1.39, 1.61, and 3.33 [2 H, $4 \mathrm{H}$, and $2 \mathrm{H}, 3 \times \mathrm{m}, \mathrm{OCH}_{2}\left(\mathrm{CH}_{2}\right)_{4} \mathrm{~N}_{3}$ ], 2.01 and $2.02($ each $3 \mathrm{H}, 2 \times \mathrm{s}$, $2 \times \mathrm{NAc}), 3.34$ (1 H, br t, H-2"), 3.44 (1 H, br t, H-4"), 3.45 (1 $\mathrm{H}$, br t, $\left.\mathrm{H}-3^{\prime \prime}\right), 3.58$ and 3.88 [each $1 \mathrm{H}, 2 \times \mathrm{m}, \mathrm{OCH}_{2}\left(\mathrm{CH}_{2}\right)_{4} \mathrm{~N}_{3}$ ], 
$3.62\left(1 \mathrm{H}, \mathrm{m}, \mathrm{H}-5^{\prime \prime}\right), 3.68\left(1 \mathrm{H}, \mathrm{m}, \mathrm{H}-5^{\prime}\right), 3.72\left(1 \mathrm{H}, \mathrm{dd}, J_{\mathrm{H}-2^{\prime}, \mathrm{H}-3}\right.$ $10.8 \mathrm{~Hz}, J_{\mathrm{H}-3^{\prime}, \mathrm{H}-4^{\prime}} 3.4 \mathrm{~Hz}, \mathrm{H}-3^{\prime}$ ), 3.76 and 3.80 (each $1 \mathrm{H}, 2 \times \mathrm{m}$, $\left.2 \times \mathrm{H}^{-} 6^{\prime}\right), 3.78$ and 4.05 (each $\left.1 \mathrm{H}, 2 \times \mathrm{m}, 2 \times \mathrm{H}-6\right), 3.82(1 \mathrm{H}$, m, H-5), $3.84\left(1 \mathrm{H}, \mathrm{dd}, J_{\mathrm{H}-2, \mathrm{H}-3} 10.9, J_{\mathrm{H}-3, \mathrm{H}-4} 3.2, \mathrm{H}-3\right), 3.89(1 \mathrm{H}$, br t, H-2'), $3.93(1 \mathrm{H}$, br d, H-4' $), 3.98\left(1 \mathrm{H}, \mathrm{dd}, J_{\mathrm{H}-1, \mathrm{H}-2} 8.6, \mathrm{H}-\right.$ 2), $4.16\left(1 \mathrm{H}\right.$, br d, H-4), $4.17\left(1 \mathrm{H}, \mathrm{dd}, J_{\mathrm{H}-5^{\prime \prime}, \mathrm{H}-6 \mathrm{~b}^{\prime \prime}} 5.8, J_{\mathrm{H}-6 \mathrm{a}^{\prime \prime}, \mathrm{H}-6 \mathrm{~b}^{\prime \prime}}\right.$ $\left.11.3, \mathrm{H}-6 \mathrm{~b}^{\prime \prime}\right), 4.30\left(1 \mathrm{H}, \mathrm{dd}, J_{\mathrm{H}-5^{\prime \prime}, \mathrm{H}-6 \mathrm{a}^{\prime \prime}} 2.1, \mathrm{H}-6 \mathrm{a}^{\prime \prime}\right), 4.45(1 \mathrm{H}, \mathrm{d}$, $\mathrm{H}-1), 4.46$ (1 H, d, $\left.J_{\mathrm{H}-1^{\prime}, \mathrm{H}-2^{\prime}} 8.4, \mathrm{H}-1^{\prime}\right), 4.50\left(1 \mathrm{H}, \mathrm{d}, J_{\mathrm{H}-1^{\prime \prime}, \mathrm{H}-2^{\prime \prime}} 7.8\right.$, $\left.\mathrm{H}-1^{\prime \prime}\right) ; \delta_{\mathrm{C}}\left(125 \mathrm{MHz} ; \mathrm{D}_{2} \mathrm{O}\right) 22.9\left(\mathrm{NDCOCH}_{3}\right), 23.2,28.4,28.8$, and $51.8\left[\mathrm{OCH}_{2}\left(\mathrm{CH}_{2}\right)_{4} \mathrm{~N}_{3}\right], 51.8(\mathrm{C}-2), 53.1\left(\mathrm{C}-2^{\prime}\right), 61.7\left(\mathrm{C}-6^{\prime}\right)$, $67.9\left(\mathrm{C}-6^{\prime \prime}\right), 68.5\left(\mathrm{C}-4^{\prime}\right), 69.0(\mathrm{C}-4), 70.1\left(\mathrm{C}-3^{\prime \prime}\right), 70.5(\mathrm{C}-6), 70.7$ $\left[\mathrm{OCH}_{2}\left(\mathrm{CH}_{2}\right)_{4} \mathrm{~N}_{3}\right], 71.7\left(\mathrm{C}-3^{\prime}\right), 73.5\left(\mathrm{C}-2^{\prime \prime}\right), 74.2(\mathrm{C}-5), 74.3(\mathrm{C}-$ $\left.5^{\prime \prime}\right), 75.8\left(\mathrm{C}-5^{\prime}\right), 76.2$ (C-4"), 80.7 (C-3), 101.8 (C-1), 102.6 (C$\left.1^{\prime}\right), 104.9\left(\mathrm{C}-1^{\prime \prime}\right)$; high resolution MALDI-TOF MS, $\mathrm{m} / \mathrm{z}$ found $\mathrm{M}+\mathrm{Na} 822.229, \mathrm{C}_{27} \mathrm{H}_{46} \mathrm{~N}_{5} \mathrm{Na}_{2} \mathrm{O}_{19} \mathrm{~S}$ requires 822.230.

5-Aminopentyl (2-acetamido-2-deoxy- $\beta$-D-galactopyranosyl)$(1 \rightarrow 6)$-[(sodium $\beta$-D-glucopyranosyl 6-sulfate)-( $\rightarrow 3)]-2$ acetamido-2-deoxy- $\beta$-D-galactopyranoside 5

A solution of $36(5 \mathrm{mg}, 6.3 \mu \mathrm{mol})$ in $0.05 \mathrm{M}$ aq. $\mathrm{NaOH}\left(1.0 \mathrm{~cm}^{3}\right)$ was added dropwise to a suspension of $10 \% \mathrm{Pd}-\mathrm{C}(0.9 \mathrm{mg})$ and $\mathrm{NaHB}_{4}(4.0 \mathrm{mg})$ in water $\left(0.5 \mathrm{~cm}^{3}\right)$. The suspension was stirred for $1 \mathrm{~h}$, when TLC $(6: 2.5: 1.5 \mathrm{EtOAc}-\mathrm{MeOH}$-water $)$ showed the disappearance of $\mathbf{3 6}$. After filtration through Celite, size-

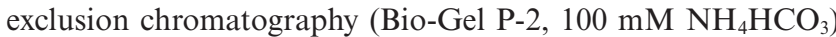
gave $5(4.3 \mathrm{mg}, 89 \%)$, isolated after lyophilization from water, as a white, amorphous powder; $[\alpha]_{\mathrm{D}}^{20}+2$ (c 0.1 in water); $\delta_{\mathrm{H}}\left(500 \mathrm{MHz} ; \mathrm{D}_{2} \mathrm{O} ; 2 \mathrm{D}\right.$ TOCSY and HSQC) $1.42,1.60,1.67$, and 2.98 [each $2 \mathrm{H}, 4 \times \mathrm{m}, \mathrm{OCH}_{2}\left(\mathrm{CH}_{2}\right)_{4} \mathrm{ND}_{2}$ ], 2.01 and 2.02 (each $3 \mathrm{H}, 2 \times \mathrm{s}, 2 \times \mathrm{NAc}), 3.31\left(1 \mathrm{H}\right.$, br t, $\left.\mathrm{H}-2^{\prime \prime}\right), 3.43(1 \mathrm{H}$, br t, H-4"), 3.45 (1 H, br t, H-3"), 3.59 and 3.88 [each $1 \mathrm{H}, 2 \times \mathrm{m}$, $\mathrm{OCH}_{2}\left(\mathrm{CH}_{2}\right)_{4} \mathrm{ND}_{2}$ ], 3.62 (1 H, m, H-5"), 3.67 (1 H, m, H-5'), 3.72 $\left(1 \mathrm{H}, \mathrm{dd}, J_{\mathrm{H}-2^{\prime}, \mathrm{H}-3^{\prime}} 11.0 \mathrm{~Hz}, J_{\mathrm{H}-3^{\prime}, \mathrm{H}-4^{\prime}} 3.5 \mathrm{~Hz}, \mathrm{H}-3^{\prime}\right), 3.75$ and 3.80 (each $\left.1 \mathrm{H}, 2 \times \mathrm{m}, 2 \times \mathrm{H}^{\prime} 6^{\prime}\right), 3.76(1 \mathrm{H}, \mathrm{m}, \mathrm{H}-5), 3.82$ and 4.04 (each $1 \mathrm{H}, 2 \times \mathrm{m}, 2 \times \mathrm{H}-6), 3.83\left(1 \mathrm{H}, \mathrm{dd}, J_{\mathrm{H}-2, \mathrm{H}-3} 10.6, J_{\mathrm{H}-3, \mathrm{H}-4} 3.4\right.$, $\mathrm{H}-3), 3.90$ (1 H, br t, H-2'), $3.93(1 \mathrm{H}$, br d, H-4'), $3.98(1 \mathrm{H}$, dd, $\left.J_{\mathrm{H}-1, \mathrm{H}-2} 8.4, \mathrm{H}-2\right), 4.16\left(1 \mathrm{H}\right.$, br d, H-4), $4.17\left(1 \mathrm{H}, \mathrm{dd}, J_{\mathrm{H}-5^{\prime \prime}, \mathrm{H}-6 \mathrm{~b}^{\prime}}\right.$ $\left.6.0, J_{\mathrm{H}-6 \mathrm{a}^{\prime \prime}, \mathrm{H}-6 \mathrm{~b}^{\prime \prime}} 11.1, \mathrm{H}-6 \mathrm{~b}^{\prime \prime}\right), 4.31\left(1 \mathrm{H}, \mathrm{dd}, J_{\mathrm{H}-5^{\prime \prime}, \mathrm{H}-6 \mathrm{a}^{\prime \prime}} 1.8, \mathrm{H}-6 \mathrm{a}^{\prime \prime}\right)$, $4.44(1 \mathrm{H}, \mathrm{d}, \mathrm{H}-1), 4.46\left(1 \mathrm{H}, \mathrm{d}, J_{\mathrm{H}-1^{\prime}, \mathrm{H}-2^{\prime}} 8.5, \mathrm{H}-1^{\prime}\right), 4.49(1 \mathrm{H}, \mathrm{d}$, $\left.J_{\mathrm{H}-1^{\prime \prime}, \mathrm{H}-2^{\prime \prime}} 8.0, \mathrm{H}-1^{\prime \prime}\right) ; \delta_{\mathrm{C}}\left(125 \mathrm{MHz} ; \mathrm{D}_{2} \mathrm{O}\right) 22.7,27.1,28.7$, and 40.0 $\left[\mathrm{OCH}_{2}\left(\mathrm{CH}_{2}\right)_{4} \mathrm{ND}_{2}\right], 22.8$ and $22.9\left(2 \times \mathrm{NDCOCH}_{3}\right), 51.8(\mathrm{C}-2)$, $53.1\left(\mathrm{C}-2^{\prime}\right), 61.7\left(\mathrm{C}-6^{\prime}\right), 67.9\left(\mathrm{C}-6^{\prime \prime}\right), 68.5\left(\mathrm{C}-4^{\prime}\right), 69.0(\mathrm{C}-4), 70.1$ (C-4"), $70.4\left[\mathrm{OCH}_{2}\left(\mathrm{CH}_{2}\right)_{4} \mathrm{ND}_{2}\right], 70.5(\mathrm{C}-6), 71.7\left(\mathrm{C}-3^{\prime}\right), 73.4(\mathrm{C}-$ $\left.2^{\prime \prime}\right), 74.2$ (C-5), 74.3 (C-5"), 75.8 (C-5'), 76.2 (C-3"), 80.6 (C-3), $101.9(\mathrm{C}-1), 102.6\left(\mathrm{C}-1^{\prime}\right), 104.8\left(\mathrm{C}-1^{\prime \prime}\right)$; high resolution MALDITOF MS, $m / z$ found $\mathrm{M}+\mathrm{Na} 796.233, \mathrm{C}_{27} \mathrm{H}_{48} \mathrm{~N}_{3} \mathrm{Na}_{2} \mathrm{O}_{19} \mathrm{~S}$ requires 796.240.

\section{4-Methoxyphenyl (6- $O$-levulinoyl-2,3,4-tri- $O$-p-toluoyl- $\beta$-D- glucopyranosyl)-(1 $\rightarrow 3$ )-4,6-di- $O$-acetyl-2-deoxy-2-phthalimido- $\beta$-D-galactopyranoside 37}

To $24(0.75 \mathrm{~g}, 0.57 \mathrm{mmol})$ was added a $1 \mathrm{M}$ TBAF solution in THF $\left(15 \mathrm{~cm}^{3}\right)$, before use neutralized at $0{ }^{\circ} \mathrm{C}$ with HOAc. The mixture was stirred for $1 \mathrm{~h}$ at $0{ }^{\circ} \mathrm{C}$ and overnight at $\mathrm{rt}$, when TLC (95: $5 \mathrm{CH}_{2} \mathrm{Cl}_{2}$-acetone) showed the disappearance of $\mathbf{2 4}$ and the formation of a new product $\left(R_{\mathrm{f}}=0.29\right)$. After concentration, a solution of the residue in EtOAc was washed with water and $10 \%$ aq. $\mathrm{NaCl}$, dried, filtered, and concentrated. Column chromatography (95:5 $\mathrm{CH}_{2} \mathrm{Cl}_{2}$-acetone) of the residue gave the free HO6 intermediate isolated as a yellow foam. A solution of the free HO6 intermediate $(0.54 \mathrm{~g}, 0.50 \mathrm{mmol})$ in $1: 1$ pyridine-acetic anhydride $\left(20 \mathrm{~cm}^{3}\right)$ was stirred overnight, when TLC (95:5 $\mathrm{CH}_{2} \mathrm{Cl}_{2}$-acetone) showed the reaction to be complete $\left(R_{\mathrm{f}}=0.79\right)$. After co-concentration with toluene, a solution of the residue in $\mathrm{CH}_{2} \mathrm{Cl}_{2}$ was washed with saturated aq. $\mathrm{NaHCO}_{3}$ and $10 \%$ aq. $\mathrm{NaCl}$, dried, filtered, and concentrated. Column chromatography (95:5 $\mathrm{CH}_{2} \mathrm{Cl}_{2}$-acetone) of the residue gave $37(0.53 \mathrm{~g}, 84 \%)$, isolated as a yellow foam; $[\alpha]_{\mathrm{D}}^{20}+31(c 0.5 \mathrm{in}$ $\left.\mathrm{CHCl}_{3}\right) ; \delta_{\mathrm{H}}\left(500 \mathrm{MHz} ; \mathrm{CDCl}_{3} ; 2 \mathrm{D}\right.$ TOCSY and HSQC) 2.07,
2.20, 2.24, 2.26, and $2.33[3 \mathrm{H}, 3 \mathrm{H}, 3 \mathrm{H}, 3 \mathrm{H}$, and $6 \mathrm{H}, 5 \times \mathrm{s}$, $3 \times \mathrm{COC}_{6} \mathrm{H}_{4} \mathrm{CH}_{3}, 2 \times \mathrm{COCH}_{3}$, and $\left.\mathrm{CO}\left(\mathrm{CH}_{2}\right)_{2} \mathrm{COCH}_{3}\right], 2.63$ and 2.77 [each $2 \mathrm{H}, 2 \times \mathrm{m}, \mathrm{CO}\left(\mathrm{CH}_{2}\right)_{2} \mathrm{COCH}_{3}$ ], $3.68(3 \mathrm{H}, \mathrm{s}$, $\left.\mathrm{C}_{6} \mathrm{H}_{4} \mathrm{OCH}_{3}\right), 3.91\left(1 \mathrm{H}, \mathrm{m}, \mathrm{H}-5^{\prime}\right), 4.10(1 \mathrm{H}, \mathrm{m}, \mathrm{H}-5), 4.26$ $\left(1 \mathrm{H}, \mathrm{dd}, J_{\mathrm{H}-5, \mathrm{H}-6 \mathrm{a}} 4.3 \mathrm{~Hz}, J_{\mathrm{H}-6 \mathrm{a}, \mathrm{H}-6 \mathrm{~b}} 11.1 \mathrm{~Hz}, \mathrm{H}-6 \mathrm{a}\right), 4.38$ (1 H, dd, $\left.J_{\mathrm{H}-5^{\prime}, \mathrm{H}-6 \mathrm{a}^{\prime}} 2.8, J_{\mathrm{H}-6 \mathrm{a}^{\prime}, \mathrm{H}-6 \mathrm{~b}^{\prime}} 13.6, \mathrm{H}-6 \mathrm{a}^{\prime}\right), 4.74\left(1 \mathrm{H}, \mathrm{dd}, J_{\mathrm{H}-1, \mathrm{H}-2} 8.5\right.$, $\left.J_{\mathrm{H}-2, \mathrm{H}-3} 11.3, \mathrm{H}-2\right), 4.80\left(1 \mathrm{H}, \mathrm{d}, J_{\mathrm{H}-1^{\prime}, \mathrm{H}-2^{\prime}} 7.8, \mathrm{H}-1^{\prime}\right), 4.93(1 \mathrm{H}, \mathrm{dd}$, $\left.J_{\mathrm{H}-3, \mathrm{H}-4} 3.4, \mathrm{H}-3\right), 5.27\left(1 \mathrm{H}, \mathrm{dd}, J_{\mathrm{H}-2^{\prime}, \mathrm{H}-3^{\prime}} 10.0, \mathrm{H}-2^{\prime}\right), 5.42(1 \mathrm{H}, \mathrm{br}$ t, H-4'), 5.48 (1 H, d, H-1), $5.64(1 \mathrm{H}$, br t, H-3'), $5.66(1 \mathrm{H}$, br d, H-4), 6.66 and 6.74 (each $2 \mathrm{H}, 2 \times \mathrm{m}, \mathrm{C}_{6} \mathrm{H}_{4} \mathrm{OCH}_{3}$ ), 6.88, 6.99, 7.13, 7.37, 7.54, and 7.73 (each $2 \mathrm{H}, 6 \times \mathrm{d}, 3 \times \mathrm{COC}_{6} \mathrm{H}_{4} \mathrm{CH}_{3}$ ); $\delta_{\mathrm{C}}\left(125 \mathrm{MHz} ; \mathrm{CDCl}_{3}\right) 20.9,21.1,21.6,21.7(2 \mathrm{C})$, and 29.8 $\left[3 \times \mathrm{COC}_{6} \mathrm{H}_{4} \mathrm{CH}_{3}, 2 \times \mathrm{COCH}_{3}\right.$, and $\left.\mathrm{CO}\left(\mathrm{CH}_{2}\right)_{2} \mathrm{COCH}_{3}\right], 28.0$ and $38.1\left[\mathrm{CO}\left(\mathrm{CH}_{2}\right)_{2} \mathrm{COCH}_{3}\right], 52.4(\mathrm{C}-2), 55.7\left(\mathrm{C}_{6} \mathrm{H}_{4} \mathrm{OCH}_{3}\right), 62.2$ $\left(\mathrm{C}-6^{\prime}\right), 62.7(\mathrm{C}-6), 68.9\left(\mathrm{C}-4^{\prime}\right), 69.2(\mathrm{C}-4), 71.7\left(\mathrm{C}-2^{\prime}\right), 71.9(\mathrm{C}-$ 5), 72.3 (C-5'), 72.5 (C-3'), 74.6 (C-3), 98.0 (C-1), 101.6 (C-1'), 114.5 and $118.6\left(C_{6} \mathrm{H}_{4} \mathrm{OCH}_{3}\right), 123.6$ and $133.8\left[\mathrm{~N}(\mathrm{CO})_{2} C_{6} \mathrm{H}_{4}\right]$, $128.9,129.0,129.2,129.7,129.8$, and $130.0\left(\mathrm{COC}_{6} \mathrm{H}_{4} \mathrm{CH}_{3}\right)$; high resolution MALDI-TOF MS, $m / z$ found $\mathrm{M}+\mathrm{Na} 1136.365$, $\mathrm{C}_{60} \mathrm{H}_{59} \mathrm{NNaO}_{20}$ requires 1136.353.

(6- $O$-Levulinoyl-2,3,4-tri- $O$-p-toluoyl- $\beta$-D-glucopyranosyl)-(1 $\rightarrow$ 3)-4,6-di- $O$-acetyl-2-deoxy-2-phthalimido- $\beta$-D-galactopyranosyl trichloroacetimidate 38

To a solution of $37(0.52 \mathrm{~g}, 0.47 \mathrm{mmol})$ in $1: 1: 1$ tolueneacetonitrile-water $\left(30 \mathrm{~cm}^{3}\right)$ was added ammonium cerium(IV) nitrate $(2.56 \mathrm{~g}, 4.7 \mathrm{mmol})$. The two-phase mixture was stirred for $2 \mathrm{~h}$, when TLC (95:5 $\mathrm{CH}_{2} \mathrm{Cl}_{2}$-acetone) showed the disappearance of 37. The mixture was diluted with EtOAc, and the organic phase was washed with saturated aq. $\mathrm{NaHCO}_{3}$ and $10 \%$ aq. $\mathrm{NaCl}$, dried, filtered, and concentrated. Column chromatography (95:5 $\mathrm{CH}_{2} \mathrm{Cl}_{2}$-acetone) of the residue gave the hemiacetal intermediate, isolated as an orange foam. To a solution of the hemiacetal $(0.38 \mathrm{~g}, 0.38 \mathrm{mmol})$ in dry $\mathrm{CH}_{2} \mathrm{Cl}_{2}$ $\left(3 \mathrm{~cm}^{3}\right)$ and trichloroacetonitrile $\left(0.41 \mathrm{~cm}^{3}, 3.8 \mathrm{mmol}\right)$ was added, at $0{ }^{\circ} \mathrm{C}, 1,8$-diazabicyclo[5.4.0]undec-7-ene $\left(6.7 \mathrm{~mm}^{3}\right.$, $38 \mu \mathrm{mol})$. The reaction was stirred for $16 \mathrm{~h}$, then concentrated. Column chromatography ( $95: 5 \mathrm{CH}_{2} \mathrm{Cl}_{2}$-acetone) of the residue yielded $38(0.32 \mathrm{~g}, 58 \%)$, isolated as a yellow foam; $[\alpha]_{\mathrm{D}}^{20}+29\left(c 1\right.$ in $\left.\mathrm{CHCl}_{3}\right) ; \delta_{\mathrm{H}}\left(500 \mathrm{MHz} ; \mathrm{CDCl}_{3} ; 2 \mathrm{D}\right.$ TOCSY and HSQC) $2.08,2.21,2.24,2.28,2.32$, and 2.33 [each $3 \mathrm{H}, 6 \times \mathrm{s}$, $3 \times \mathrm{COC}_{6} \mathrm{H}_{4} \mathrm{CH}_{3}, 2 \times \mathrm{COCH}_{3}$, and $\left.\mathrm{CO}\left(\mathrm{CH}_{2}\right)_{2} \mathrm{COCH}_{3}\right], 2.63$ and 2.80 [each $2 \mathrm{H}, 2 \times \mathrm{m}, \mathrm{CO}\left(\mathrm{CH}_{2}\right)_{2} \mathrm{COCH}_{3}$ ], $3.92(1 \mathrm{H}, \mathrm{m}$, H-5'), 4.11 (1 H, dd, H-6b), 4.14 (1 H, dd, H-6b'), 4.19 (1 H, br t, H-5), 4.29 (1 H, dd, $\left.J_{\mathrm{H}-5, \mathrm{H}-6 \mathrm{a}} 5.1 \mathrm{~Hz}, J_{\mathrm{H}-6 \mathrm{a}, \mathrm{H}-6 \mathrm{~b}} 11.3 \mathrm{~Hz}, \mathrm{H}-6 \mathrm{a}\right)$, $4.39\left(1 \mathrm{H}, \mathrm{dd}, J_{\mathrm{H}-5^{\prime}, \mathrm{H}-6 \mathrm{a}^{\prime}} 2.6, J_{\mathrm{H}-6 \mathrm{a}^{\prime}, \mathrm{H}-6 \mathrm{~b}^{\prime}} 12.1, \mathrm{H}-6 \mathrm{a}^{\prime}\right), 4.76(1 \mathrm{H}$, $\left.\mathrm{dd}, J_{\mathrm{H}-1, \mathrm{H}-2} 9.0, J_{\mathrm{H}-2, \mathrm{H}-3} 11.3, \mathrm{H}-2\right), 4.80\left(1 \mathrm{H}, \mathrm{d}, J_{\mathrm{H}-1^{\prime}, \mathrm{H}-2^{\prime}} 7.8, \mathrm{H}-\right.$ $\left.1^{\prime}\right), 5.01\left(1 \mathrm{H}, \mathrm{dd}, J_{\mathrm{H}-3, \mathrm{H}-4} 3.4, \mathrm{H}-3\right), 5.27\left(1 \mathrm{H}, \mathrm{dd}, J_{\mathrm{H}-2^{\prime}, \mathrm{H}-3^{\prime}} 9.9\right.$, H-2'), 5.43 (1 H, br t, H-4'), 5.65 (1 H, br t, H-3'), $5.69(1 \mathrm{H}$, br d, H-4), 6.29 (1 H, d, H-1), 6.87, 6.99, 7.13, 7.36, 7.56, and $7.74\left(\right.$ each $\left.2 \mathrm{H}, 6 \times \mathrm{d}, 3 \times \mathrm{COC}_{6} \mathrm{H}_{4} \mathrm{CH}_{3}\right), 8.42\left[\mathrm{OC}(\mathrm{N} H) \mathrm{CCl}_{3}\right]$; $\delta_{\mathrm{C}}\left(125 \mathrm{MHz} ; \mathrm{CDCl}_{3}\right) 20.8,21.0,21.6,21.7(2 \mathrm{C})$, and 29.8 $\left[3 \times \mathrm{COC}_{6} \mathrm{H}_{4} \mathrm{CH}_{3}, 2 \times \mathrm{COCH}_{3}\right.$, and $\left.\mathrm{CO}\left(\mathrm{CH}_{2}\right)_{2} \mathrm{COCH}_{3}\right], 28.0$ and $38.1\left[\mathrm{CO}\left(\mathrm{CH}_{2}\right)_{2} \mathrm{COCH}_{3}\right], 51.4(\mathrm{C}-2), 62.2\left(\mathrm{C}-6^{\prime}\right), 62.3(\mathrm{C}-$ 6), 68.9 (C-4'), 69.0 (C-4), $71.6\left(\mathrm{C}-2^{\prime}\right), 72.3\left(\mathrm{C}-5^{\prime}\right), 72.4$ (C-3'), 72.7 (C-5), 74.1 (C-3), 94.4 (C-1), $101.5\left(\mathrm{C}-1^{\prime}\right), 123.3$ and 133.8 $\left[\mathrm{N}(\mathrm{CO})_{2} \mathrm{C}_{6} \mathrm{H}_{4}\right], 128.9,129.1,129.2,129.6,129.8$, and 130.0 $\left(\mathrm{COC}_{6} \mathrm{H}_{4} \mathrm{CH}_{3}\right)$.

\section{5-Azidopentyl (6-O-levulinoyl-2,3,4-tri- $O$-p-toluoyl- $\beta$-D-glucopyranosyl)-( $\rightarrow 3)-(4,6-d i-O$-acetyl-2- deoxy-2-phthalimido- $\beta$-D-galactopyranosyl)-( $1 \rightarrow$ 6)-[(6- $O$-levulinoyl-2,3,4-tri- $O$ - $p$-toluoyl- $\beta$-D-glucopyranosyl)- $(1 \rightarrow 3) \mid-4-O$-acetyl-2-deoxy-2-phthalimido- $\beta$-D- galactopyranoside 39}

A solution of $27(74 \mathrm{mg}, 68 \mu \mathrm{mol})$ and $\mathbf{3 8}(117.3 \mathrm{mg}, 102 \mu \mathrm{mol})$ in dry $\mathrm{CH}_{2} \mathrm{Cl}_{2}\left(3 \mathrm{~cm}^{3}\right)$, containing activated molecular sieves ( $4 \AA, 0.1 \mathrm{~g}$ ), was stirred for $45 \mathrm{~min}$ at $\mathrm{rt}$, then TMSOTf $\left(2.0 \mathrm{~mm}^{3}, 10.2 \mu \mathrm{mol}\right)$ was added at $0{ }^{\circ} \mathrm{C}$. The mixture was 
stirred for $15 \mathrm{~min}$, when TLC $\left(9: 1 \mathrm{CH}_{2} \mathrm{Cl}_{2}\right.$-acetone) showed the formation of 39 to be complete $\left(R_{\mathrm{f}}=0.41\right)$. After neutralization with pyridine and filtration, the solution was washed with $10 \%$ aq. $\mathrm{NaCl}$, dried, filtered, and concentrated. Column chromatography (9:1 $\mathrm{CH}_{2} \mathrm{Cl}_{2}$-acetone) of the residue rendered $39(100 \mathrm{mg}, 70 \%)$, isolated as a glass; $[\alpha]_{\mathrm{D}}^{20}+3\left(c 1\right.$ in $\left.\mathrm{CHCl}_{3}\right)$; $\delta_{\mathrm{H}}\left(500 \mathrm{MHz} ; \mathrm{CDCl}_{3} ; 2 \mathrm{D}\right.$ TOCSY and HSQC) $0.84,1.00,1.12$, and 2.74 [each $2 \mathrm{H}, 4 \times \mathrm{m}, \mathrm{OCH}_{2}\left(\mathrm{CH}_{2}\right)_{4} \mathrm{~N}_{3}$ ], 2.12, 2.14, 2.20, $2.21,2.22,2.23,2.24,2.30,2.31$, and $2.33[3 \mathrm{H}, 3 \mathrm{H}, 3 \mathrm{H}, 3 \mathrm{H}$, $3 \mathrm{H}, 3 \mathrm{H}, 3 \mathrm{H}, 3 \mathrm{H}, 3 \mathrm{H}$, and $6 \mathrm{H}, 10 \times \mathrm{s}, 6 \times \mathrm{COC}_{6} \mathrm{H}_{4} \mathrm{CH}_{3}$, $3 \times \mathrm{COCH}_{3}$, and $2 \times \mathrm{CO}\left(\mathrm{CH}_{2}\right)_{2} \mathrm{COCH}_{3}$ ], 2.57 and 2.75 [each $4 \mathrm{H}, 2 \times \mathrm{m}, 2 \times \mathrm{CO}\left(\mathrm{CH}_{2}\right)_{2} \mathrm{COCH}_{3}$ ], 2.78 and 3.14 [each $1 \mathrm{H}$, $\left.2 \times \mathrm{m}, \mathrm{OCH}_{2}\left(\mathrm{CH}_{2}\right)_{4} \mathrm{~N}_{3}\right], 3.34\left(1 \mathrm{H}, \mathrm{dd}, J_{\mathrm{H}-5, \mathrm{H}-6 \mathrm{~b}} 8.7 \mathrm{~Hz}, J_{\mathrm{H}-6 \mathrm{a}, \mathrm{H}-6 \mathrm{~b}}\right.$ $10.9 \mathrm{~Hz}, \mathrm{H}-6 \mathrm{~b}), 3.77$ (1 H, m, H-5), 3.84 (1 H, m, H-5"'), 3.91 (1 H, m, H-5"), 3.93 (1 H, m, H-6a), $3.99\left(1 \mathrm{H}, \mathrm{m}, \mathrm{H}-5^{\prime}\right), 4.08$ and 4.22 (each $\left.1 \mathrm{H}, 2 \times \mathrm{m}, 2 \times \mathrm{H}-6^{\prime}\right), 4.13$ and 4.24 (each $1 \mathrm{H}$, $2 \times$ br t, $\left.2 \times \mathrm{H}-6^{\prime \prime \prime}\right), 4.15\left(1 \mathrm{H}, \mathrm{dd}, J_{\mathrm{H}-5^{\prime \prime}, \mathrm{H}-6 \mathrm{~b}^{\prime \prime}} 5.2, J_{\mathrm{H}-6 \mathrm{a}^{\prime \prime}, \mathrm{H}-6 \mathrm{~b}^{\prime \prime}} 12.2\right.$, $\left.\mathrm{H}-6 \mathrm{~b}^{\prime \prime}\right), 4.32\left(1 \mathrm{H}, \mathrm{dd}, J_{\mathrm{H}-1, \mathrm{H}-2} 8.6, J_{\mathrm{H}-2, \mathrm{H}-3} 11.3, \mathrm{H}-2\right), 4.36(1 \mathrm{H}$, $\left.\mathrm{dd}, J_{\mathrm{H}-5^{\prime \prime}, \mathrm{H}-6 \mathrm{a}^{\prime \prime}} 2.1, \mathrm{H}-6 \mathrm{a}^{\prime \prime}\right), 4.46\left(1 \mathrm{H}, \mathrm{dd}, J_{\mathrm{H}-1^{\prime}, \mathrm{H}-2^{\prime}} 8.4, J_{\mathrm{H}-2^{\prime}, \mathrm{H}-3^{\prime}} 11.2\right.$, $\left.\mathrm{H}-2^{\prime}\right), 4.67\left(1 \mathrm{H}, \mathrm{dd}, J_{\mathrm{H}-3, \mathrm{H}-4} 3.2, \mathrm{H}-3\right), 4.71(2 \mathrm{H}, \mathrm{br} \mathrm{d}, \mathrm{H}-1$ and $\left.\mathrm{H}-1^{\prime \prime \prime}\right), 4.76\left(1 \mathrm{H}, \mathrm{d}, J_{\mathrm{H}-1^{\prime \prime}, \mathrm{H}-2^{\prime \prime}} 7.8, \mathrm{H}-1^{\prime \prime}\right), 4.85\left(1 \mathrm{H}, \mathrm{dd}, J_{\mathrm{H}-3^{\prime}, \mathrm{H}-4^{\prime}} 3.2\right.$, $\left.\mathrm{H}-3^{\prime}\right), 5.02\left(1 \mathrm{H}, \mathrm{d}, \mathrm{H}-1^{\prime}\right), 5.20\left(1 \mathrm{H}, \mathrm{dd}, J_{\mathrm{H}-1^{\prime \prime \prime}, \mathrm{H}-2^{\prime \prime}} 7.9, J_{\mathrm{H}-2^{\prime \prime \prime}, \mathrm{H}-3^{\prime \prime \prime}} 9.9\right.$, $\left.\mathrm{H}-2^{\prime \prime \prime}\right), 5.23\left(1 \mathrm{H}, \mathrm{dd}, J_{\mathrm{H}-2^{\prime \prime}, \mathrm{H}-3^{\prime \prime}} 10.0, \mathrm{H}-2^{\prime \prime}\right), 5.37$ (1 H, br t, H-4"'), $5.40(1 \mathrm{H}$, br t, H-4"), $5.41(1 \mathrm{H}$, br d, H-4), $5.59(1 \mathrm{H}$, br t, H$\left.3^{\prime \prime \prime}\right), 5.61(1 \mathrm{H}$, br t, H-3"'), $5.62(1 \mathrm{H}$, br d, H-4'), 6.83, 6.84, 6.96, $6.98,7.11,7.12,7.29,7.31,7.52,7.53,7.71$, and 7.73 (each $2 \mathrm{H}$, $\left.12 \times \mathrm{d}, 6 \times \mathrm{COC}_{6} \mathrm{H}_{4} \mathrm{CH}_{3}\right) ; \delta_{\mathrm{C}}\left(125 \mathrm{MHz} ; \mathrm{CDCl}_{3}\right) 20.8,20.9,21.0$ $(2 \mathrm{C}), 21.6(2 \mathrm{C}), 21.7(3 \mathrm{C})$, and $29.9(2 \mathrm{C})\left[6 \times \mathrm{COC}_{6} \mathrm{H}_{4} \mathrm{CH}_{3}\right.$, $3 \times \mathrm{COCH}_{3}$, and $\left.2 \times \mathrm{CO}\left(\mathrm{CH}_{2}\right)_{2} \mathrm{COCH}_{3}\right], 23.0,28.3,29.8$, and $51.1\left[\mathrm{OCH}_{2}\left(\mathrm{CH}_{2}\right)_{4} \mathrm{~N}_{3}\right], 27.9$ and $38.1\left[2 \times \mathrm{CO}\left(\mathrm{CH}_{2}\right)_{2} \mathrm{COCH}_{3}\right]$, $52.4\left(\mathrm{C}-2^{\prime}\right), 52.5(\mathrm{C}-2), 62.3\left(\mathrm{C}-6^{\prime \prime}\right), 62.4\left(\mathrm{C}-6^{\prime \prime \prime}\right), 62.6\left(\mathrm{C}-6^{\prime}\right), 68.5$ $\left[\mathrm{OCH}_{2}\left(\mathrm{CH}_{2}\right)_{4} \mathrm{~N}_{3}\right], 68.8(\mathrm{C}-6), 68.9\left(\mathrm{C}-4^{\prime \prime \prime}\right), 69.0\left(\mathrm{C}-4^{\prime \prime}\right), 69.3(\mathrm{C}-$ $\left.4^{\prime}\right), 69.8(\mathrm{C}-4), 71.6\left(\mathrm{C}-5^{\prime}\right), 71.7$ (2 C) (C-2" and C-2"'), 72.2 (2 C) (C-5" and C-5"'), 72.5 (2 C) (C-3" and C-3"'), 73.7 (C-5), $74.5(\mathrm{C}-$ 3'), 75.0 (C-3), 98.3 (C-1), $98.6\left(\mathrm{C}-1^{\prime}\right), 101.4$ (2 C) (C-1" and C$\left.1^{\prime \prime \prime}\right), 123.1,123.3$, and $133.7\left[\mathrm{~N}(\mathrm{CO})_{2} C_{6} \mathrm{H}_{4}\right], 128.9,129.0,129.2$, 129.6, 129.8, and $130.0\left(\mathrm{COC}_{6} \mathrm{H}_{4} \mathrm{CH}_{3}\right)$; high resolution MALDITOF MS, $m / z$ found $\mathrm{M}+\mathrm{Na} 2088.691, \mathrm{C}_{109} \mathrm{H}_{111} \mathrm{~N}_{5} \mathrm{NaO}_{36}$ requires 2088.691.

\section{5-Azidopentyl $(2,3,4$-tri- $O$ - $p$-toluoyl- $\beta$-D- glucopyranosyl)-( $\rightarrow 3)-(4,6$-di- $O$-acetyl-2- \\ deoxy-2-phthalimido- $\beta$-D-galactopyranosyl)-( $1 \rightarrow$ 6)-[(2,3,4-tri- $O$ - $p$-toluoyl- $\beta$-D-glucopyranosyl)-(1 $\rightarrow 3)]-4-O$ - acetyl-2-deoxy-2-phthalimido- $\beta$-D-galactopyranoside 40}

To a solution of $39(90 \mathrm{mg}, 43 \mu \mathrm{mol})$ in $\mathrm{EtOH}\left(10 \mathrm{~cm}^{3}\right)$ and toluene $\left(3 \mathrm{~cm}^{3}\right)$ was added hydrazine acetate $(20 \mathrm{mg}, 215 \mu \mathrm{mol})$. The mixture was stirred for $2 \mathrm{~h}$, then concentrated. Column chromatography $\left(9: 1 \mathrm{CH}_{2} \mathrm{Cl}_{2}\right.$-acetone) of the residue yielded 40 $(60 \mathrm{mg}, 75 \%)$, isolated as a white glass; $[\alpha]_{\mathrm{D}}^{20}+5\left(c 0.2\right.$ in $\left.\mathrm{CHCl}_{3}\right)$; $\delta_{\mathrm{H}}\left(500 \mathrm{MHz} ; \mathrm{CDCl}_{3} ; 2 \mathrm{D}\right.$ TOCSY and HSQC) $0.88,1.05,1.12$, and 2.72 [each $2 \mathrm{H}, 4 \times \mathrm{m}, \mathrm{OCH}_{2}\left(\mathrm{CH}_{2}\right)_{4} \mathrm{~N}_{3}$ ] $, 2.11,2.20,2.22$, $2.27,2.28,2.31$, and $2.33(3 \mathrm{H}, 3 \mathrm{H}, 6 \mathrm{H}, 3 \mathrm{H}, 3 \mathrm{H}, 3 \mathrm{H}$, and $6 \mathrm{H}$, $7 \times \mathrm{s}, 6 \times \mathrm{COC}_{6} \mathrm{H}_{4} \mathrm{CH}_{3}$ and $3 \times \mathrm{COCH}_{3}$ ), 2.89 and 3.21 [each $\left.1 \mathrm{H}, 2 \times \mathrm{m}, \mathrm{OCH}_{2}\left(\mathrm{CH}_{2}\right)_{4} \mathrm{~N}_{3}\right], 3.43\left(1 \mathrm{H}, \mathrm{dd}, J_{\mathrm{H}-5, \mathrm{H}-6 \mathrm{~b}} 8.4 \mathrm{~Hz}\right.$, $\left.J_{\mathrm{H}-6 \mathrm{a}, \mathrm{H}-6 \mathrm{~b}} 10.7 \mathrm{~Hz}, \mathrm{H}-6 \mathrm{~b}\right), 3.56\left(1 \mathrm{H}, \mathrm{dd}, J_{\mathrm{H}-5^{\prime \prime \prime}, \mathrm{H}-6 \mathrm{~b}^{\prime \prime}} 6.1, J_{\mathrm{H}-6 \mathrm{a}^{\prime \prime}, \mathrm{H}-6 \mathrm{~b} "}\right.$ $\left.12.3, \mathrm{H}-6 \mathrm{~b}^{\prime \prime \prime}\right), 3.60\left(1 \mathrm{H}, \mathrm{dd}, J_{\mathrm{H}-5^{\prime \prime}, \mathrm{H}-6 \mathrm{~b}^{\prime \prime}} 6.4, J_{\mathrm{H}-6 \mathrm{a}^{\prime \prime}, \mathrm{H}-6 \mathrm{~b}^{\prime \prime}} 12.9, \mathrm{H}-6 \mathrm{~b}^{\prime \prime}\right)$, 3.71 (2 H, m, H-6a" and H-6a"'), 3.73 (1 H, m, H-5"'), $3.76(1 \mathrm{H}$, m, H-5), 3.79 (1 H, m, H-5"), 3.88 (1 H, dd, $\left.J_{\mathrm{H}-5, \mathrm{H}-6 \mathrm{a}} 2.1, \mathrm{H}-6 \mathrm{a}\right)$, $4.00\left(1 \mathrm{H}, \mathrm{m}, \mathrm{H}-5^{\prime}\right), 4.15\left(2 \mathrm{H}, \mathrm{m}, 2 \times \mathrm{H}^{-} 6^{\prime}\right), 4.33\left(1 \mathrm{H}, \mathrm{dd}, J_{\mathrm{H}-1, \mathrm{H}-2}\right.$ $\left.8.6, J_{\mathrm{H}-2, \mathrm{H}-3} 11.2, \mathrm{H}-2\right), 4.50\left(1 \mathrm{H}, \mathrm{dd}, J_{\mathrm{H}-1^{\prime}, \mathrm{H}-2^{\prime}} 8.6, J_{\mathrm{H}-2^{\prime}, \mathrm{H}-3^{\prime}} 11.1\right.$, $\left.\mathrm{H}-2^{\prime}\right), 4.65\left(1 \mathrm{H}, \mathrm{dd}, J_{\mathrm{H}-3, \mathrm{H}-4} 3.5, \mathrm{H}-3\right), 4.71(1 \mathrm{H}, \mathrm{d}, \mathrm{H}-1), 4.79$ $\left(1 \mathrm{H}, \mathrm{d}, J_{\mathrm{H}-1^{\prime \prime}, \mathrm{H}-2^{\prime \prime \prime}} 8.0, \mathrm{H}-1^{\prime \prime \prime}\right), 4.82\left(1 \mathrm{H}, \mathrm{dd}, J_{\mathrm{H}-3^{\prime}, \mathrm{H}-4^{\prime}} 3.6, \mathrm{H}-3^{\prime}\right)$, $4.86\left(1 \mathrm{H}, \mathrm{d}, J_{\mathrm{H}-1^{\prime \prime}, \mathrm{H}-2^{\prime \prime}} 7.8, \mathrm{H}-1^{\prime \prime}\right), 5.02\left(1 \mathrm{H}, \mathrm{d}, \mathrm{H}-1^{\prime}\right), 5.21(1 \mathrm{H}$, dd, $\left.J_{\mathrm{H}-2^{\prime \prime}, \mathrm{H}-3^{\prime \prime}} 10.0, \mathrm{H}-2^{\prime \prime \prime}\right), 5.25\left(1 \mathrm{H}, \mathrm{dd}, J_{\mathrm{H}-2^{\prime \prime}, \mathrm{H}-3^{\prime \prime}} 9.9, \mathrm{H}-2^{\prime \prime}\right), 5.31$ (1 H, br t, H-4"'), 5.33 (1 H, br t, H-4"), 5.53 (1 H, br d, H-4), 5.63 (1 H, br t, H-3"'), $5.65(1 \mathrm{H}$, br t, H-3"), $5.72(1 \mathrm{H}$, br d, H-4'), $6.75,6.78,6.94,7.13,7.27,7.50,7.51,7.74$, and $7.75(2 \mathrm{H}, 2 \mathrm{H}$, $4 \mathrm{H}, 4 \mathrm{H}, 4 \mathrm{H}, 2 \mathrm{H}, 2 \mathrm{H}, 2 \mathrm{H}$, and $\left.2 \mathrm{H}, 9 \times \mathrm{d}, 6 \times \mathrm{COC}_{6} H_{4} \mathrm{CH}_{3}\right)$; $\delta_{\mathrm{C}}\left(125 \mathrm{MHz} ; \mathrm{CDCl}_{3}\right)$ 20.7, 21.2, 21.3, $21.6(4 \mathrm{C})$, and $21.8(2 \mathrm{C})$ $\left(6 \times \mathrm{COC}_{6} \mathrm{H}_{4} \mathrm{CH}_{3}\right.$ and $\left.3 \times \mathrm{COCH}_{3}\right), 22.9,28.3,29.7$, and 51.1 $\left[\mathrm{OCH}_{2}\left(\mathrm{CH}_{2}\right)_{4} \mathrm{~N}_{3}\right], 52.3\left(\mathrm{C}-2^{\prime}\right), 52.5(\mathrm{C}-2), 61.4\left(\mathrm{C}-6^{\prime \prime \prime}\right), 61.5(\mathrm{C}-$ $\left.6^{\prime \prime}\right), 61.9\left(\mathrm{C}-6^{\prime}\right), 68.2(\mathrm{C}-6), 68.6\left[\mathrm{OCH}_{2}\left(\mathrm{CH}_{2}\right)_{4} \mathrm{~N}_{3}\right], 68.7$ (2 C) (C$4^{\prime \prime}$ and C-4"'), $69.3\left(\mathrm{C}-4^{\prime}\right), 70.1(\mathrm{C}-4), 71.2\left(\mathrm{C}-5^{\prime}\right), 71.7$ (2 C) (C-2" and $\left.\mathrm{C}-2^{\prime \prime \prime}\right), 72.7$ (2 C) (C-3" and C-3"'), 73.4 (C-5), 75.3 (C-5"), 75.4 (C-5'"'), 75.8 (C-3'), 75.9 (C-3), 98.3 (C-1), $98.4\left(\mathrm{C}-1^{\prime}\right), 102.0$ (C-1"'), $102.2\left(\mathrm{C}-1^{\prime \prime}\right), 123.0,123.3$, and $133.5\left[\mathrm{~N}(\mathrm{CO})_{2} C_{6} \mathrm{H}_{4}\right]$, 128.9, 129.0, 129.2, 129.6, 129.8, and $130.0\left(\mathrm{COC}_{6} \mathrm{H}_{4} \mathrm{CH}_{3}\right)$; high resolution MALDI-TOF MS, $\mathrm{m} / \mathrm{z}$ found $\mathrm{M}+\mathrm{Na} 1892.647$, $\mathrm{C}_{99} \mathrm{H}_{99} \mathrm{~N}_{5} \mathrm{NaO}_{32}$ requires 1892.617.

\section{5-Azidopentyl (sodium $\beta$-D-glucopyranosyl 6-sulfate)-( $\rightarrow 3$ )- (2-acetamido-2-deoxy- $\beta$-D-galactopyranosyl)-( $1 \rightarrow 6)-[($ sodium $\beta$-D-glucopyranosyl 6-sulfate)-( $\rightarrow 3)]$-2-acetamido-2-deoxy- $\beta$-D- galactopyranoside 42}

To a solution of $40(53 \mathrm{mg}, 28 \mu \mathrm{mol})$ in $\operatorname{DMF}\left(3 \mathrm{~cm}^{3}\right)$ was added the sulfur trioxide trimethylamine complex $(157 \mathrm{mg}$, $1.12 \mathrm{mmol})$. The mixture was stirred for $48 \mathrm{~h}$ at $50{ }^{\circ} \mathrm{C}$, when TLC (9: $\left.1 \mathrm{CH}_{2} \mathrm{Cl}_{2}-\mathrm{MeOH}\right)$ showed the complete conversion of 40 into non-sodiated $41\left(R_{\mathrm{f}}=0.16\right)$. After quenching the reaction with $\mathrm{MeOH}\left(10 \mathrm{~cm}^{3}\right)$, the solution was co-concentrated with toluene. A solution of the residue in $\operatorname{EtOAc}\left(50 \mathrm{~cm}^{3}\right)$ was washed with saturated aq. $\mathrm{NaHCO}_{3}$ and $10 \%$ aq. $\mathrm{NaCl}$, dried, filtered, and concentrated. The residue was dissolved in $\mathrm{MeOH}\left(10 \mathrm{~cm}^{3}\right)$, containing Dowex $50 \mathrm{~W}$ X $8 \mathrm{Na}^{+}$resin, and stirred for $15 \mathrm{~min}$, then filtered and concentrated. Column chromatography $(9: 1$ $\mathrm{CH}_{2} \mathrm{Cl}_{2}-\mathrm{MeOH}$ ) of the residue gave 41 (45 mg, 77\%), isolated as a white, amorphous powder; $\delta_{\mathrm{H}}\left(300 \mathrm{MHz} ; \mathrm{CDCl}_{3}\right) 0.90$, 1.13 , and $2.82\left[2 \mathrm{H}, 4 \mathrm{H}\right.$, and $\left.2 \mathrm{H}, 3 \times \mathrm{m}, \mathrm{OCH}_{2}\left(\mathrm{CH}_{2}\right)_{4} \mathrm{~N}_{3}\right], 2.21$, $2.23,2.25,2.30$, and $2.34(3 \mathrm{H}, 3 \mathrm{H}, 6 \mathrm{H}, 6 \mathrm{H}$, and $9 \mathrm{H}, 5 \times \mathrm{s}$, $6 \times \mathrm{COC}_{6} \mathrm{H}_{4} \mathrm{CH}_{3}$ and $3 \times \mathrm{COCH}_{3}$ ), 3.01 and 3.35 [each $1 \mathrm{H}$, $\left.2 \times \mathrm{m}, \mathrm{OCH}_{2}\left(\mathrm{CH}_{2}\right)_{4} \mathrm{~N}_{3}\right], 3.52\left(1 \mathrm{H}, \mathrm{dd}, J_{\mathrm{H}-5, \mathrm{H}-6 \mathrm{~b}} 8.4 \mathrm{~Hz}, J_{\mathrm{H}-6 \mathrm{a}, \mathrm{H}-6 \mathrm{~b}}\right.$ $10.5 \mathrm{~Hz}, \mathrm{H}-6 \mathrm{~b}), 3.84$ (1 H, dd, $\left.J_{\mathrm{H}-5, \mathrm{H}-6 \mathrm{a}} 2.0, \mathrm{H}-6 \mathrm{a}\right), 3.92(1 \mathrm{H}, \mathrm{m}$, $\left.\mathrm{H}-5^{\prime}\right), 4.42\left(1 \mathrm{H}, \mathrm{dd}, J_{\mathrm{H}-1^{\prime}, \mathrm{H}-2^{\prime}} 8.5, J_{\mathrm{H}-2^{\prime}, \mathrm{H}-3^{\prime}} 11.2, \mathrm{H}-2^{\prime}\right), 4.75(1 \mathrm{H}$, $\left.\mathrm{d}, J_{\mathrm{H}-1^{\prime \prime \prime}, \mathrm{H}-2^{\prime \prime \prime}} 7.8, \mathrm{H}-1^{\prime \prime \prime}\right), 4.78\left(1 \mathrm{H}, \mathrm{d}, J_{\mathrm{H}-1, \mathrm{H}-2} 8.5, \mathrm{H}-1\right), 4.81(1 \mathrm{H}, \mathrm{d}$, $\left.J_{\mathrm{H}-1^{\prime \prime}, \mathrm{H}-2^{\prime}} 7.7, \mathrm{H}-1^{\prime \prime}\right), 4.92\left(1 \mathrm{H}, \mathrm{dd}, J_{\mathrm{H}-2^{\prime}, \mathrm{H}-3^{\prime}} 11.3, J_{\mathrm{H}-3^{\prime}, \mathrm{H}-4^{\prime}} 3.2, \mathrm{H}-3^{\prime}\right)$, $5.14\left(1 \mathrm{H}, \mathrm{d}, \mathrm{H}-1^{\prime}\right), 5.18\left(1 \mathrm{H}, \mathrm{dd}, J_{\mathrm{H}-2^{\prime \prime \prime} \cdot \mathrm{H}-3^{\prime \prime}} 9.6, \mathrm{H}-2^{\prime \prime \prime}\right), 5.22(1 \mathrm{H}$, dd, $\left.J_{\mathrm{H}-2^{\prime \prime}, \mathrm{H}-3^{\prime \prime}} 9.5, \mathrm{H}-2^{\prime \prime}\right), 5.34(1 \mathrm{H}$, br t, H-4"'), 5.37 (1 H, br t, H$\left.4^{\prime \prime}\right), 5.58\left(1 \mathrm{H}\right.$, br d, $\left.J_{\mathrm{H}-3, \mathrm{H}-4} 3.4, \mathrm{H}-4\right), 5.63(1 \mathrm{H}$, br t, H-3"'), 5.67 $(1 \mathrm{H}$, br t, H-3"), $5.80(1 \mathrm{H}$, br d, H-4'), 6.87, 6.90, 7.00, 7.15, 7.31, 7.33, 7.54, and $7.73(2 \mathrm{H}, 2 \mathrm{H}, 4 \mathrm{H}, 4 \mathrm{H}, 2 \mathrm{H}, 2 \mathrm{H}, 4 \mathrm{H}$, and $\left.4 \mathrm{H}, 8 \times \mathrm{s}, 6 \times \mathrm{COC}_{6} \mathrm{H}_{4} \mathrm{CH}_{3}\right)$.

A solution of $41(35 \mathrm{mg}, 22 \mu \mathrm{mol})$ in ethanolic $33 \% \mathrm{CH}_{3} \mathrm{NH}_{2}$ $\left(5 \mathrm{~cm}^{3}\right)$ was stirred for 7 days, during which time the mixture was three times concentrated and fresh ethanolic $33 \% \mathrm{CH}_{3} \mathrm{NH}_{2}$ $\left(5 \mathrm{~cm}^{3}\right)$ was added. After co-concentration with toluene, to a solution of the residue in dry $\mathrm{MeOH}$ at $0{ }^{\circ} \mathrm{C}$ was added acetic anhydride $\left(100 \mathrm{~mm}^{3}\right)$. The mixture was stirred for $3 \mathrm{~h}$ at $0{ }^{\circ} \mathrm{C}$, then concentrated. Size-exclusion chromatography (Bio-Gel P2, $100 \mathrm{mM} \mathrm{NH}_{4} \mathrm{HCO}_{3}$ ) of the residue afforded $\mathbf{4 2}(11 \mathrm{mg}, 62 \%)$, isolated after lyophilization from water, as a white, amorphous powder; $[\alpha]_{\mathrm{D}}^{20}-11(c 0.6$ in water $) ; \delta_{\mathrm{H}}\left(500 \mathrm{MHz} ; \mathrm{D}_{2} \mathrm{O} ; 2 \mathrm{D}\right.$ TOCSY and HSQC) $1.40,1.59$, and $3.30[2 \mathrm{H}, 4 \mathrm{H}$, and $2 \mathrm{H}, 3 \times \mathrm{m}$, $\mathrm{OCH}_{2}\left(\mathrm{CH}_{2}\right)_{4} \mathrm{~N}_{3}$ ], 2.01 and 2.02 (each $\left.3 \mathrm{H}, 2 \times \mathrm{s}, 2 \times \mathrm{NAc}\right), 3.32$ (2 H, br t, H-2" and H-2"'), 3.45 (4 H, m, H-3", H-3"', H-4", and $\left.\mathrm{H}-4^{\prime \prime \prime}\right), 3.57$ and 3.88 [each $1 \mathrm{H}, 2 \times \mathrm{m}, \mathrm{OCH}_{2}\left(\mathrm{CH}_{2}\right)_{4} \mathrm{~N}_{3}$ ], 3.62 (2 H, m, H-5" and H-5"'), 3.69 (1 H, m, H-5'), 3.78 (2 H, m, $\left.2 \times \mathrm{H}^{-6}\right), 3.81$ and 4.06 (each $\left.1 \mathrm{H}, 2 \times \mathrm{m}, 2 \times \mathrm{H}-6\right), 3.82(1 \mathrm{H}$, $\mathrm{m}, \mathrm{H}-5), 3.85$ (2 H, br t, H-3 and H-3'), 3.99 (1 H, br t, H-2), $4.01\left(1 \mathrm{H}\right.$, br t, H-2'), $4.15\left(1 \mathrm{H}\right.$, br d, $\left.J_{\mathrm{H}-3, \mathrm{H}-4} 3.2 \mathrm{~Hz}, \mathrm{H}-4\right), 4.19$ and 4.30 (each $2 \mathrm{H}, 2 \times \mathrm{m}, 2 \times \mathrm{H}-6^{\prime \prime}$ and $\left.2 \times \mathrm{H}-6^{\prime \prime \prime}\right), 4.19(1 \mathrm{H}$, br d, $\left.J_{\mathrm{H}-3^{\prime}, \mathrm{H}-4^{\prime}} 3.4, \mathrm{H}-4^{\prime}\right), 4.45\left(1 \mathrm{H}, \mathrm{d}, J_{\mathrm{H}-1, \mathrm{H}-2} 8.5, \mathrm{H}-1\right), 4.50$ $\left(1 \mathrm{H}, \mathrm{d}, J_{\mathrm{H}-1^{\prime \prime \prime}, \mathrm{H}-2^{\prime \prime \prime}} 7.9, \mathrm{H}-1^{\prime \prime \prime}\right), 4.51\left(1 \mathrm{H}, \mathrm{d}, J_{\mathrm{H}-1^{\prime \prime}, \mathrm{H}-2^{\prime \prime}} 7.8, \mathrm{H}-1^{\prime \prime}\right)$, $4.53\left(1 \mathrm{H}, \mathrm{d}, J_{\mathrm{H}-1^{\prime}, \mathrm{H}-2^{\prime}} 8.5, \mathrm{H}-1^{\prime}\right) ; \delta_{\mathrm{C}}\left(125 \mathrm{MHz} ; \mathrm{D}_{2} \mathrm{O}\right) 23.0(2 \mathrm{C})$ $\left(2 \times \mathrm{NDCOCH}_{3}\right), 23.2,28.3,28.8$, and $51.8\left[\mathrm{OCH}_{2}\left(\mathrm{CH}_{2}\right)_{4} \mathrm{~N}_{3}\right]$, 51.8 (2 C) (C-2 and C-2'), 61.9 (C-6'), 67.8 (2 C) (C-6" and C$\left.6^{\prime \prime \prime}\right), 68.6\left(\mathrm{C}-4^{\prime}\right), 68.9(\mathrm{C}-4), 70.0\left(\mathrm{C}-3^{\prime \prime}\right.$ and C-3"' $), 70.3(\mathrm{C}-6), 70.6$ $\left[\mathrm{OCH}_{2}\left(\mathrm{CH}_{2}\right)_{4} \mathrm{~N}_{3}\right], 73.4(2 \mathrm{C})\left(\mathrm{C}-2^{\prime \prime}\right.$ and C-2"') $74.2(\mathrm{C}-5), 74.3(2$ C) $\left(\mathrm{C}-5^{\prime \prime}\right.$ and $\left.\mathrm{C}-5^{\prime \prime \prime}\right), 75.6\left(\mathrm{C}-5^{\prime}\right), 76.2(2 \mathrm{C})\left(\mathrm{C}-4^{\prime \prime}\right.$ and $\left.\mathrm{C}-4^{\prime \prime \prime}\right), 80.7$ (2 C) (C-3 and C-3'), 101.8 (C-1), 102.2 (C-1'), 104.9 (C-1"'), 
105.0 (C-1"); high resolution MALDI-TOF MS, $\mathrm{m} / \mathrm{z}$ found $\mathrm{M}+\mathrm{Na}$ 1086.254, $\mathrm{C}_{33} \mathrm{H}_{55} \mathrm{~N}_{5} \mathrm{Na}_{3} \mathrm{O}_{27} \mathrm{~S}_{2}$ requires 1086.222.

5-Aminopentyl (sodium $\beta$-D-glucopyranosyl 6-sulfate)-( $\rightarrow 3$ )(2-acetamido-2-deoxy- $\beta$-D-galactopyranosyl)-( $1 \rightarrow 6)$-I(sodium $\beta$-D-glucopyranosyl 6-sulfate)-(1 $\rightarrow 3) \mid$-2-acetamido-2-deoxy- $\beta$-Dgalactopyranoside 6

A solution of $42(5 \mathrm{mg}, 4.7 \mu \mathrm{mol})$ in $0.05 \mathrm{M}$ aq. $\mathrm{NaOH}\left(0.5 \mathrm{~cm}^{3}\right)$ was added dropwise to a suspension of $10 \% \mathrm{Pd}-\mathrm{C}(0.7 \mathrm{mg})$ and $\mathrm{NaHB}_{4}(2.7 \mathrm{mg})$ in water $\left(0.5 \mathrm{~cm}^{3}\right)$. The suspension was stirred for $1 \mathrm{~h}$, when TLC $(6: 2.5: 1.5 \mathrm{EtOAc}-\mathrm{MeOH}$-water $)$ showed the disappearance of $\mathbf{4 2}$. After filtration through Celite, size-exclusion chromatography (Bio-Gel P-2, $100 \mathrm{mM} \mathrm{NH}_{4} \mathrm{HCO}_{3}$ ) gave $6(3.5 \mathrm{mg}, 71 \%)$, isolated after lyophilization from water, as a white, amorphous powder; $[\alpha]_{\mathrm{D}}^{20}-4(c 0.2$ in water $) ; \delta_{\mathrm{H}}(500 \mathrm{MHz}$; $\mathrm{D}_{2} \mathrm{O} ; 2 \mathrm{D}$ TOCSY and HSQC) 1.41, 1.60, 1.66 and 2.99 [each $\left.2 \mathrm{H}, 4 \times \mathrm{m}, \mathrm{OCH}_{2}\left(\mathrm{CH}_{2}\right)_{4} \mathrm{ND}_{2}\right], 2.01(6 \mathrm{H}, \mathrm{s}, 2 \times \mathrm{NAc}), 3.31(2 \mathrm{H}$, br t, H-2" and H-2"'), 3.45 (4 H, m, H-3", H-3"', H-4", and H-4"'), 3.59 and 3.87 [each $1 \mathrm{H}, 2 \times \mathrm{m}, \mathrm{OCH}_{2}\left(\mathrm{CH}_{2}\right)_{4} \mathrm{ND}_{2}$ ], $3.62(2 \mathrm{H}, \mathrm{m}$, H-5" and H-5"'), 3.69 (1 H, m, H-5'), $3.78\left(2 \mathrm{H}, \mathrm{m}, 2 \times \mathrm{H}-6^{\prime}\right)$, 3.83 and 4.03 (each $1 \mathrm{H}, 2 \times \mathrm{m}, 2 \times \mathrm{H}-6), 3.83(1 \mathrm{H}, \mathrm{m}, \mathrm{H}-5)$, $3.84\left(2 \mathrm{H}\right.$, br t, $\mathrm{H}-3$ and $\left.\mathrm{H}-3^{\prime}\right), 3.99(1 \mathrm{H}$, br t, H-2), $4.00(1 \mathrm{H}, \mathrm{br}$ t, H-2'), $4.15\left(1 \mathrm{H}\right.$, br d, $\left.J_{\mathrm{H}-3, \mathrm{H}-4} 3.2 \mathrm{~Hz}, \mathrm{H}-4\right), 4.18$ and 4.30 (each $2 \mathrm{H}, 2 \times \mathrm{m}, 2 \times \mathrm{H}-6^{\prime \prime}$ and $\left.2 \times \mathrm{H}-6^{\prime \prime \prime}\right), 4.18\left(1 \mathrm{H}\right.$, br d, $J_{\mathrm{H}-3^{\prime}, \mathrm{H}-4^{\prime}}$ $\left.3.2, \mathrm{H}-4^{\prime}\right), 4.45\left(1 \mathrm{H}, \mathrm{d}, J_{\mathrm{H}-1, \mathrm{H}-2} 8.5, \mathrm{H}-1\right), 4.50\left(1 \mathrm{H}, \mathrm{d}, J_{\mathrm{H}-1^{\prime \prime}, \mathrm{H}-2^{\prime \prime}}\right.$ 8.0, H-1"'), 4.52 (1 H, d, $\left.J_{\mathrm{H}-1^{\prime \prime}, \mathrm{H}-2^{\prime \prime}} 8.1, \mathrm{H}-1^{\prime \prime}\right), 4.53\left(1 \mathrm{H}, \mathrm{d}, J_{\mathrm{H}-1^{\prime}, \mathrm{H}-2}\right.$ 8.6, $\left.\mathrm{H}-1^{\prime}\right) ; \delta_{\mathrm{C}}\left(125 \mathrm{MHz} ; \mathrm{D}_{2} \mathrm{O}\right) 22.9(2 \mathrm{C})\left(2 \times \mathrm{NDCOCH}_{3}\right), 22.8$, 27.0, 28.7, and $40.0\left[\mathrm{OCH}_{2}\left(\mathrm{CH}_{2}\right)_{4} \mathrm{ND}_{2}\right], 51.8(2 \mathrm{C})(\mathrm{C}-2$ and C-2' $), 61.9\left(\mathrm{C}-6^{\prime}\right), 67.9$ (2 C) (C-6" and C-6"' $), 68.6\left(\mathrm{C}-4^{\prime}\right), 68.8$ (C-4), $70.0\left(\mathrm{C}-3^{\prime \prime}\right.$ and C-3"'), $70.4(\mathrm{C}-6), 70.5\left[\mathrm{OCH}_{2}\left(\mathrm{CH}_{2}\right)_{4} \mathrm{ND}_{2}\right]$, 73.5 (2 C) (C-2" and C-2"'), 74.2 (C-5), 74.3 (2 C) (C-5" and C-5"'), $75.6\left(\mathrm{C}-5^{\prime}\right), 76.1$ (2 C) (C-4" and C-4"'), 80.5 (2 C) (C-3 and $\left.\mathrm{C}-3^{\prime}\right), 101.9$ (C-1), 102.3 (C-1'), 104.8 (C-1"'), 104.9 (C-1"); high resolution MALDI-TOF MS, $m / z$ found $\mathrm{M}+\mathrm{Na} 1060.193$, $\mathrm{C}_{33} \mathrm{H}_{57} \mathrm{~N}_{3} \mathrm{Na}_{3} \mathrm{O}_{27} \mathrm{~S}_{2}$ requires 1060.231.

3-[2- $N$-(3,4-Dione-2-ethoxycyclobutene)aminoethylthio|propyl ( $\beta$-D-glucopyranosyluronic acid)-( $1 \rightarrow 3)$-2-acetamido-2-deoxy- $\beta$ D-glucopyranoside 43

To a solution of $\mathbf{1}(1 \mathrm{mg}, 1.9 \mu \mathrm{mol})$ in $50 \mathrm{mM}$ sodium phosphate buffer $\left(100 \mathrm{~mm}^{3}, \mathrm{pH} 7.2\right)$ was added a solution of diethyl squarate $\left(0.56 \mathrm{~mm}^{3}, 3.8 \mu \mathrm{mol}\right)$ in $\mathrm{EtOH}\left(100 \mathrm{~mm}^{3}\right)$. After stirring for $16 \mathrm{~h}, \mathrm{EtOH}$ was evaporated by flushing with $\mathrm{N}_{2}$, and the residue in water was loaded on a C-18 Extract-Clean ${ }^{\mathrm{TM}}$ column. After elution of remaining 1 with water $\left(3 \times 3 \mathrm{~cm}^{3}\right), 43$ was eluted with $\mathrm{MeOH}\left(3 \times 3 \mathrm{~cm}^{3}\right)$, then concentrated in vacuo. The pure, elongated saccharide was used directly for the preparation of neoglycoconjugate BSA-1.

3-[2- $N$-(3,4-Dione-2-ethoxycyclobutene)aminoethylthio]propyl (2-acetamido-2-deoxy- $\beta$-D-glucopyranosyl)-( $1 \rightarrow 6)-[(\beta-D-$ glucopyranosyluronic acid)-( $\rightarrow 3) \mid$-2-acetamido-2-deoxy- $\beta$-Dglucopyranoside 44

To a solution of $2(1 \mathrm{mg}, 1.4 \mu \mathrm{mol})$ in $50 \mathrm{mM}$ sodium phosphate buffer $\left(100 \mathrm{~mm}^{3}, \mathrm{pH} 7.2\right)$ was added a solution of diethyl squarate $\left(0.4 \mathrm{~mm}^{3}, 2.4 \mu \mathrm{mol}\right)$ in EtOH $\left(100 \mathrm{~mm}^{3}\right)$. After stirring for $16 \mathrm{~h}$, column chromatography $(7.5: 1.5: 1.0 \mathrm{EtOAc}-\mathrm{MeOH}-$ water) of the mixture yielded $\mathbf{4 4}$, isolated as a glass. The pure, elongated saccharide was used directly for the preparation of neoglycoconjugate BSA-2.

3-[2- $N$-(3,4-Dione-2-ethoxycyclobutene)aminoethylthio|propyl $(\beta$-D-glucopyranosyluronic acid)-( $\rightarrow 3)-(2$-acetamido-2-deoxy$\beta$-D-glucopyranosyl)-( $1 \rightarrow 6)$-I( $\beta$-D-glucopyranosyluronic acid $)-$ $(1 \rightarrow 3)]$-2-acetamido-2-deoxy- $\beta$-D-glucopyranoside 45

To a solution of $3(0.8 \mathrm{mg}, 0.9 \mu \mathrm{mol})$ in $50 \mathrm{mM}$ sodium phosphate buffer $\left(100 \mathrm{~mm}^{3}, \mathrm{pH}\right.$ 7.2) was added a solution of diethyl squarate $\left(0.26 \mathrm{~mm}^{3}, 1.8 \mu \mathrm{mol}\right)$ in $\mathrm{EtOH}\left(100 \mathrm{~mm}^{3}\right)$. After stirring for $16 \mathrm{~h}$, column chromatography $(7.5: 1.5: 1.0 \mathrm{EtOAc}-$ $\mathrm{MeOH}$-water) of the mixture yielded $\mathbf{4 5}$, isolated as a glass. The pure, elongated saccharide was used directly for the preparation of neoglycoconjugate BSA-3.

5- $N$-(3,4-Dione-2-ethoxycyclobutene)aminopentyl (sodium $\beta$ D-glucopyranosyl 6-sulfate)-(1 $\rightarrow 3)$-2-acetamido-2-deoxy- $\beta$-Dgalactopyranoside 46

To a solution of $4(0.5 \mathrm{mg}, 0.9 \mu \mathrm{mol})$ in $50 \mathrm{mM}$ sodium phosphate buffer $\left(100 \mathrm{~mm}^{3}, \mathrm{pH} 7.2\right)$ was added a solution of diethyl squarate $\left(0.26 \mathrm{~mm}^{3}, 3.6 \mu \mathrm{mol}\right)$ in $\mathrm{EtOH}\left(100 \mathrm{~mm}^{3}\right)$. After stirring for $16 \mathrm{~h}, \mathrm{EtOH}$ was evaporated by flushing with $\mathrm{N}_{2}$, and the residue in water was loaded on a C-18 Extract-Clean ${ }^{\mathrm{TM}}$ column. After elution of remaining 4 with water $\left(3 \times 3 \mathrm{~cm}^{3}\right)$, 46 was eluted with $\mathrm{MeOH}\left(3 \times 3 \mathrm{~cm}^{3}\right)$, then concentrated in vacuo. The pure, elongated saccharide was used directly for the preparation of neoglycoconjugate BSA-4.

5- $N$-(3,4-Dione-2-ethoxycyclobutene)aminopentyl (2acetamido-2-deoxy- $\beta$-D-galactopyranosyl)-( $1 \rightarrow 6)-[($ sodium $\beta$-D-glucopyranosyl 6-sulfate)-(1 $\rightarrow 3) \mid-2$-acetamido-2-deoxy- $\beta$-Dgalactopyranoside 47

To a solution of $\mathbf{5}(1.0 \mathrm{mg}, 1.2 \mu \mathrm{mol})$ in $50 \mathrm{mM}$ sodium phosphate buffer $\left(100 \mathrm{~mm}^{3}, \mathrm{pH}\right.$ 7.2) was added a solution of diethyl squarate $\left(0.4 \mathrm{~mm}^{3}, 2.4 \mu \mathrm{mol}\right)$ in $\mathrm{EtOH}\left(100 \mathrm{~mm}^{3}\right)$. After stirring for $16 \mathrm{~h}$, column chromatography $(7.5: 1.5: 1.0 \mathrm{EtOAc}-\mathrm{MeOH}-$ water) of the mixture yielded $\mathbf{4 7}$, isolated as a glass. The pure, elongated saccharide was used directly for the preparation of neoglycoconjugate BSA-5.

\section{5- $N$-(3,4-Dione-2-ethoxycyclobutene)aminopentyl (sodium} $\beta$-D-glucopyranosyl 6-sulfate)-( $\rightarrow 3)$-(2-acetamido-2-deoxy$\beta$-D-galactopyranosyl)-( $\rightarrow$ 6)-[(sodium $\beta$-D-glucopyranosyl 6sulfate)-( $1 \rightarrow 3)]$-2-acetamido-2-deoxy- $\beta$-D-galactopyranoside 48

To a solution of $6(1.0 \mathrm{mg}, 1.0 \mu \mathrm{mol})$ in $50 \mathrm{mM}$ sodium phosphate buffer $\left(100 \mathrm{~mm}^{3}, \mathrm{pH} 7.2\right)$ was added a solution of diethyl squarate $\left(0.28 \mathrm{~mm}^{3}, 2.0 \mu \mathrm{mol}\right)$ in EtOH $\left(100 \mathrm{~mm}^{3}\right)$. After stirring for $16 \mathrm{~h}$, column chromatography $(7.5: 1.5: 1.0$ EtOAc$\mathrm{MeOH}$-water) of the mixture yielded 48, isolated as a glass. The pure, elongated saccharide was used directly for the preparation of neoglycoconjugate BSA-6.

General procedure for the conjugation of elongated saccharides 43-48 to BSA

For a target oligosaccharide incorporation of about $15 \mathrm{~mol} \mathrm{~mol}^{-1}$ BSA, to a solution of an elongated saccharide (43-48, 10 equiv. based on BSA) in $0.1 \mathrm{M} \mathrm{NaHCO}_{3}$ buffer $\left(0.5 \mathrm{mg} \mathrm{cm}^{-3}, \mathrm{pH} 9.0\right)$ was added a solution of pre-treated BSA ${ }^{11}\left(20 \mathrm{mg} \mathrm{cm}^{-3}\right)$ in $0.1 \mathrm{M}$ $\mathrm{NaHCO}_{3}$ buffer. After stirring for 3-5 days, the mixtures were loaded on to a $30 \mathrm{kDa}$ Nalgene centrifugal filter, and washed with water $\left(6 \times 15 \mathrm{~cm}^{3}\right)$. After lyophilization from water, the degree of incorporation of saccharides $\mathbf{4 3 - 4 8}$ in neoglycoconjugates BSA-1-BSA-6, respectively, was determined by MALDI-TOF MS analysis. Samples $\left(0.1 \mathrm{mg} \mathrm{cm}^{-3} 1: 1\right.$ acetonitrile-water $)$ were mixed on the target plate in a ratio of $1: 1$ with the matrix 3,5dimethoxy-4-hydroxycinnamic acid $\left(10 \mathrm{mg} \mathrm{cm}^{-3}\right)$ in $1: 1$ acetonitrile-water containing $0.1 \%$ TFA.

\section{References}

1 R. F. Sturrock, 'The parasites and their life cycles', in Human Schistosomiasis, P. Jordan, G. Webbe and R. F. Sturrock, ed., CAB International, Wallingford, 1993, pp. 1-32.

2 L. Chitsulo, D. Engels, A. Montresor and L. Savioli, Acta Trop., 2000, 77, 41-51.

3 A. M. Deelder, Z. L. Qian, P. G. Kremsner, L. Acosta, A. L. T. Rabello and P. Enyong, Trop. Geogr. Med., 1994, 46, 223-238.

4 N. de Jonge, P. de Caluwé, G. W. Hilberath, F. W. Krijger, A. M. Polderman and A. M. Deelder, Trans. R. Soc. Trop. Med. Hyg., 1989, 83, 368-372. 
5 M. S. Nourel Din, R. Nibbeling, J. P. Rotmans, A. M. Polderman, F. W. Krijger and A. M. Deelder, Am. J. Trop. Med. Hyg., 1994, 50, $585-594$.

6 L. van Lieshout, A. M. Polderman and A. M. Deelder, Acta Trop., 2000, 77, 69-80.

7 I. S. Barsoum, D. G. Colley and K. A. Kamal, Exp. Parasitol., 1990, 71, 107-113.

8 A. M. Agnew, A. J. C. Fulford, N. de Jonge, F. W. Krijger, M. Rodriguez-Chacon, V. Gutsmann and A. M. Deelder, Parasitology, 1995, 111, 67-76.

9 F. W. Krijger, L. van Lieshout and A. M. Deelder, Acta Trop., 1994, 56, 55-63.

10 A. A. Bergwerff, G. J. van Dam, J. P. Rotmans, A. M. Deelder, J. P. Kamerling and J. F. G. Vliegenthart, J. Biol. Chem., 1994, 269, $31510-31517$

11 H. J. Vermeer, K. M. Halkes, J. A. van Kuik, J. P. Kamerling and J. F. G. Vliegenthart, J. Chem. Soc., Perkin Trans. 1, 2000, 2249-2263.

12 H. J. Vermeer, G. J. van Dam, K. M. Halkes, J. P. Kamerling, J. F. G. Vliegenthart, C. H. Hokke and A. M. Deelder, Parasitol. Res., 2003, 90, 330-336.

13 F. A. W. Koeman, J. W. G. Meissner, H. R. P. van Ritter, J. P. Kamerling and J. F. G. Vliegenthart, J. Carbohydr. Chem., 1994, 13, $1-25$.

14 C. Coutant and J.-C. Jacquinet, J. Chem. Soc., Perkin Trans. 1, 1995, 1573-1581.

15 K. M. Halkes, H. J. Vermeer, T. M. Slaghek, P. A. V. van Hooft, A. Loof, J. P. Kamerling and J. F. G. Vliegenthart, Carbohydr. Res., 1998, 309, 175-188.

16 O. Kanie, S. C. Crawley, M. M. Palcic and O. Hindsgaul, Carbohydr. Res., 1993, 243, 139-164.
17 M. S. Motawia, J. Wengel, A. E.-S. Abdel-Megid and E. B. Pedersen, Synthesis, 1989, 384-387.

18 F.-I. Auzanneau and B. M. Pinto, Bioorg. Med. Chem., 1996, 4 2003-2010.

19 R. R. Schmidt, J. Michel and M. Roos, Liebigs Ann. Chem., 1984, 1343-1357.

20 T. M. Slaghek, Y. Nakahara, T. Ogawa, J. P. Kamerling and J. F. G. Vliegenthart, Carbohydr. Res., 1994, 255, 61-85.

21 T. Fukuyama, A. A. Laird and L. M. Hotchkiss, Tetrahedron Lett., 1985, 26, 6291-6292.

22 T. Nakano, Y. Ito and T. Ogawa, Tetrahedron Lett., 1990, 31, 1597-1600.

23 J. A. F. Joosten, J. P. Kamerling and J. F. G. Vliegenthart, Carbohydr. Res., 2003, 338, 2611-2627.

24 A. Carvalho de Souza, K. M. Halkes, J. D. Meeldijk, A. J. Verkleij, J. F. G. Vliegenthart and J. P. Kamerling, Eur. J. Org. Chem., 2004, in press.

25 J. H. van Boom and P. M. J. Burgers, Tetrahedron Lett., 1976 , 4875-4879.

26 N. Jeker and C. Tamm, Helv. Chim. Acta, 1988, 71, 1895-1903.

27 H. J. Vermeer, J. P. Kamerling and J. F. G. Vliegenthart, Tetrahedron: Asymmetry, 2000, 11, 539-547.

28 P. B. van Seeventer, M. A. Corsten, M. P. Sanders, J. P. Kamerling and J. F. G. Vliegenthart, Carbohydr. Res., 1997, 299, $171-179$.

29 L. F. Tietze, C. Schröter, S. Gabius, U. Brinck, A. Goerlach-Graw and H.-J. Gabius, Bioconjugate Chem., 1991, 2, 148-153.

30 L. F. Tietze, M. Arlt, M. Beller, K.-H. Glüsenkamp, E. Jähde and M. F. Rajewski, Chem. Ber., 1991, 124, 1215-1221.

31 D. J. Lefeber, J. P. Kamerling and J. F. G. Vliegenthart, Chem. Eur. J., 2001, 7, 4411-4421. 